\title{
SINGULAR ESTIMATES AND UNIFORM STABILITY OF COUPLED SYSTEMS OF HYPERBOLIC/PARABOLIC PDES
}

\author{
F. BUCCI, I. LASIECKA, AND R. TRIGGIANI
}

Received 30 October 2001

\section{Statement of the problem. Main results}

1.1. Introduction; singular estimates for coupled PDE systems. The present paper sets itself along that line of research established by [1, 3] - and later streamlined and simplified in the exposition of [16] - that is focused on mathematical properties of an accepted acoustic chamber model $[8,31]$, subject to unbounded control action on its flexible wall.

We consider a generalization of an established structural acoustic model. This consists of a coupled system of two partial differential equations of different types - a hyperbolic PDE acting within the acoustic chamber and a paraboliclike PDE acting on the elastic wall of the chamber-which are strongly coupled at their common interface. Unlike prior literature, we allow the parameter $\alpha-$ which measures the strength of damping in the plate-like component-to run over the entire range $1 / 2 \leq \alpha \leq 1$ of analyticity (parabolicity) of its free dynamics. Prior literature considered only the limit case $\alpha=1$ ("Kelvin Voight damping"). However, the limit case $\alpha=1 / 2$ ("structural, square root damping") is at least equally important; indeed, even more so in applications. Entirely new phenomena arise over the case $\alpha=1$ as $\alpha$ decreases from 1 to $1 / 2$, which we describe below. Indeed, as $\alpha$ decreases from 1 to $1 / 2$, we seek to achieve two critical control-theoretic properties of the overall coupled system: (i) a quantitatively precise singular estimate for the operator $e^{A t} B$ as $t \searrow 0$ and, simultaneously, (ii) a uniform (exponential) stability result of the free dynamics operator $e^{A t}$ (which is not analytic, of course). Here, $B$ is the unbounded control operator: it typically contains derivatives of Dirac measures supported on points or curves of the elastic wall of the chamber. It turns out that goals (i) and (ii) are in conflict with one another, as $\alpha$ decreases from 1 to $1 / 2$. Indeed, as the damping strength $\alpha$ of the plate (parabolic) component decreases from 1 to $1 / 2$, a progressively stronger 
damping on the boundary of the wave (hyperbolic) component is needed in order to achieve goal (i). However, beyond an explicitly computed threshold of $\alpha$, then the additional boundary damping on the wave introduces instability of the overall free dynamics $e^{A t}$, thus violating goal (ii). This occurs since, beyond a certain threshold of $\alpha$, excessive wave damping-which is essential to achieve goal (i) - causes, however, the origin (and only the origin) to become a point in the continuous spectrum of the overall dynamic operator $A$. In order to remove this pathology, and hence obtain both goals (i) and (ii) simultaneously beyond a certain threshold of $\alpha$, an additional "static damping" (acting on the position) is introduced in the boundary conditions of the wave component. The paper gives a precise description of these two intertwined and conflicting aspects for the purpose of achieving the simplest possible model, as a function of $\alpha, 1 / 2 \leq \alpha \leq 1$, where both goals (i) and (ii) are attained. These then insure, as a consequence, a rich optimal control theory of the corresponding control problem.

The case $\alpha=1$; hence $D_{0}=0 ; \beta=0$, in (1.2c)-(1.2d) below (see $[1,3,16]$ ). In the aforementioned references, the original model consisted of a coupled system of two partial differential equations (PDEs) of different type-a hyperbolic PDE and a parabolic-like PDE-which are strongly coupled at the interface (represented by the flexible wall $\left.\Gamma_{0}\right)$. More precisely, the original model in the analysis of $[1,3,16]$ is problem (1.1) below with constant $\alpha=1$ in (1.2d) and, hence, damping operator $D_{0}$ taken to be null: $D_{0}=0$, while the constant $\beta$ is taken equal to zero: $\beta=0$ in $(1.2 \mathrm{c})$. In the resulting specialization of model (1.2), a wave equation in $z$ (a hyperbolic PDE, problems (1.2a), (1.2b), and (1.2c)) which is active within the chamber, influences through its velocity trace $\left.z_{t}\right|_{\Gamma_{0}}$ on the flexible wall $\Gamma_{0}$ the dynamics of a plate-like abstract Euler-Bernoulli equation (1.2d) in $v$ defined on $\Gamma_{0}$ and subject to (strong) Kelvin-Voight damping (a parabolic PDE with $\rho>0, \alpha=1)$. In turn, the velocity $v_{t}$ of the plate deflection acts on the boundary conditions $(1.2 \mathrm{c})$ of the wave equation on the flexible wall $\Gamma_{0}$. The Kelvin-Voight damping of these aforementioned works corresponds to the value $\alpha=1$ and $\rho>0$ of the two constants entering the model in (1.2d) below, with, further, $\beta=0$, and $D_{0}=0$, as already pointed out. For this model, these references first settle the preliminary (and, in this case, rather standard) question of well-posedness of the overall PDE coupled system, by asserting the existence of a strongly continuous contraction semigroup $e^{A t}$ in the resulting energy space $Y$ (see $(2.11)$ below with $\beta=0$ ). Then, these references $[1,3,16]$ establish the following key, fundamental result. Let $B$ be the resulting unbounded control operator acting on the overall model (see (2.39) below) and expressed in terms of the control operator $\mathscr{B}$ acting on the plate equation in $(1.2 \mathrm{~d})$, then the term $e^{A t} B$ satisfies, in the uniform operator norm of $Y$, a singular estimate of the type

$$
\left\|e^{A t} B\right\|=O_{T}\left(\frac{1}{t^{\gamma}}\right), \quad 0<t \leq T, 0<\gamma<1
$$


Here $\gamma$ is a constant, which is explicitly identified in terms of the degree of unboundedness - measured by the parameter $0<r<1 / 2$ in (1.3) —of the control operator $\mathscr{B}$ below, which acts on the plate equation $(1.2 \mathrm{~d})$.

To really appreciate the singular estimate (1.1), it should be emphasized that the strongly continuous (s.c.) well-posedness semigroup $e^{A t}$ referred to above, is not analytic on $Y$. Yet, the above singular estimate (1.1) is a measure that reveals and emphasizes that parabolicity_originally present only in the plate component on the flexible wall $\Gamma_{0}$ - has actually propagated onto the entire overall chamber model.

Consequences of the singular estimate (1.1). Achieving the singular estimate (1.1) for coupled PDE systems such as those arising in the structural acoustic problem has important critical consequences. It would suffice to point out the basic impact that estimate (1.1) has on the theory of quadratic optimal control problems and corresponding differential/integral Riccati equations $(T<\infty)$ or algebraic Riccati equations $(T=\infty)$. Indeed, under the validity of estimate (1.1), and yet in the absence of analyticity of the s.c. free dynamics semigroup $e^{A t}$, one has the following results: in the case $T<\infty$ (resp., in the case $T=\infty$ ), one obtains a rather comprehensive and satisfactory theory of the quadratic optimal control problem and corresponding differential/integral Riccati equations (resp., algebraic Riccati equations) which is truly akin to that of the parabolic (analytic semigroup) case. Indeed, estimate (1.1) allows for a suitable adaptation of the parabolic (analytic semigroup) technique. In the case $T=\infty$, we require, in addition, that $e^{A t}$ is also uniformly (exponentially) stable on $Y$ in order to satisfy the finite cost condition. In particular, under (1.1), one obtains the very important consequence that the gain operator $B^{*} P(t)$ (resp., $B^{*} P$ ), where $P(t)$ (resp., $P)$ is the corresponding Riccati operator, is bounded as an operator between the state space and the control space. See $[1,3,16,18,26]$ for a progressively more comprehensive treatment. See also [35] for a treatment of the corresponding min-max problem and of the quadratic cost case with nondefinite cost. For the optimal control theory for single PDE classes, we refer to [4, 24, 25].

The case $1 / 2 \leq \alpha<1$ and its consequences on the choice of the dynamical model. The present paper maintains the hyperbolic/parabolic coupling of the aforementioned works for the overall coupled PDE system, but its goal is to enlarge the scope of the parabolic component from the original Kelvin-Voight damping $\alpha=1$ to the entire range of parabolicity $1 / 2 \leq \alpha \leq 1$ (see $[5,6]$ ) initially with $\beta=0$. Of course, the two most important cases in applications are the extreme cases $\alpha=1 / 2$ and $\alpha=1$. Stimulated by the above discussion, our objective is twofold.

(i) First, we seek to extend the singular estimate (1.1) to the entire analyticity range $1 / 2 \leq \alpha \leq 1$. In order to achieve (1.1) as $\alpha$ decreases from $\alpha=1$ to $\alpha=1 / 2$, we will be forced to introduce a progressively stronger damping term $D_{0} z_{t}$ in the Boundary Conditions (BC) of the wave component in (1.2c), up to 
a second-order tangential operator $D_{0}$ for $\alpha=1 / 2$ (see Theorem 1.7). This way, with $1 / 2 \leq \alpha \leq 1$ and $\beta=0$, the singular estimate (1.1) is established and thus optimal control theory in a finite horizon is covered as an extension of the parabolic case (see [26]).

(ii) The damping operator $D_{0}$ imposed in step (i) in order to achieve the beneficial singular estimate (1.1) introduces, however, negative effects on the stability of the wave component (let alone, of the overall system) by introducing the origin $\lambda=0$ as a point in the continuous spectrum of the relevant dynamics operator of the wave component and of the overall system, even in the presence of viscous damping on the entire domain $\left(d_{2}>0\right.$ in $\left.(1.2 \mathrm{a})\right)$. This is shown in Section 4. One natural way to see this state of affairs is to say that an original model with $d_{2}=0$ is not uniformly stabilizable by a choice of viscous damping $d_{2}>0$. Thus, with this model, even with $d_{2}>0$, the optimal control theory over an infinite time horizon is out of reach, as the preliminary finite cost condition is not verified. Therefore, in order to recover the optimal control theory on $T=\infty$ by requiring, in addition, exponential (uniform) stabilization of $e^{A t}$, one is then forced to further modify the original coupled PDE model, by introducing this time a comparable (stabilizer) boundary term $\beta D_{0} z$ with $\beta>0$ on $\Gamma_{0}$ in (1.2c), whose purpose is to eliminate the point $\lambda=0$ from the (continuous) spectrum of the overall dynamic operator. A more complete description of our strategy is delineated after the quantitative introduction of the (ultimate) model and a statement of the most relevant results.

\subsection{Mathematical model and strategy}

The mathematical model. In qualitative terms, the mathematical model under consideration consists of a wave equation, active within an acoustic chamber, which is then strongly coupled with a dynamic abstract plate equation acting only on the elastic, flat wall of the chamber. In turn, the plate equation is then subject to an unbounded control action. More precisely, let $\Omega \subset \mathbb{R}^{3}$ be an open bounded domain ("the acoustic chamber") with boundary $\Gamma=\overline{\Gamma_{0} \cup \Gamma_{1}}$, where $\Gamma_{0}$ and $\Gamma_{1}$ are open, connected, disjoint parts, $\Gamma_{0} \cap \Gamma_{1}=\emptyset$ in $\mathbb{R}^{2}$, of positive measure. The sub-boundary $\Gamma_{0}$ is flat and is referred to as the elastic or flexible wall. Instead, $\Gamma_{1}$ is referred to as the rigid or hard wall. The interaction between wave and plate takes place on $\Gamma_{0}$. We also assume that either $\Omega$ is sufficiently smooth (say, $\Gamma$ is of class $C^{2}$ ), or else $\Omega$ is convex. This assumption guarantees that solutions to classical elliptic equations with $L_{2}(\Omega)$-forcing term are in $H^{2}(\Omega)[11]$, or that the domain of the Laplacian in $\Omega$, with (either Dirichlet or) Neumann BC, is contained in $H^{2}(\Omega)$ (see (2.1) in our case). The acoustic medium in the chamber is described by the wave equation in the variable $z$ with acoustic pressure $\rho_{1} z_{t}$ where $\rho_{1}$ is the density of the fluid. Moreover, let $c^{2}$ be the speed of sound. Finally, denote by $v$ the "abstract deflection" of the abstract plate equation on $\Gamma_{0}$. Then, in this paper, we consider the following coupled PDE system: 


$$
\begin{array}{ll}
z_{t t}=c^{2} \Delta z-d_{2} z_{t}+f & \text { in } Q=(0, T] \times \Omega ; \\
\frac{\partial z}{\partial v}+d_{1} z=0 & \text { on } \Sigma_{1}=(0, T] \times \Gamma_{1} ; \\
\frac{\partial z}{\partial v}+D_{0} z_{t}+\beta D_{0} z=v_{t} & \text { on } \Sigma_{0}=(0, T] \times \Gamma_{0} ; \\
v_{t t}+A_{v}+\rho \mathscr{A}^{\alpha} v_{t}+\left.\rho_{1} z_{t}\right|_{\Gamma_{0}}=\mathscr{B} u & \text { on } \Sigma_{0}=(0, T] \times \Gamma_{0} ; \\
z(0, \cdot)=z_{0}, \quad z_{t}(0, \cdot)=z_{1} & \text { in } \Omega ; \\
v(0, \cdot)=v_{0}, \quad v_{t}(0, \cdot)=v_{1} & \text { in } \Gamma_{0},
\end{array}
$$

under the following assumptions to be held throughout the paper.
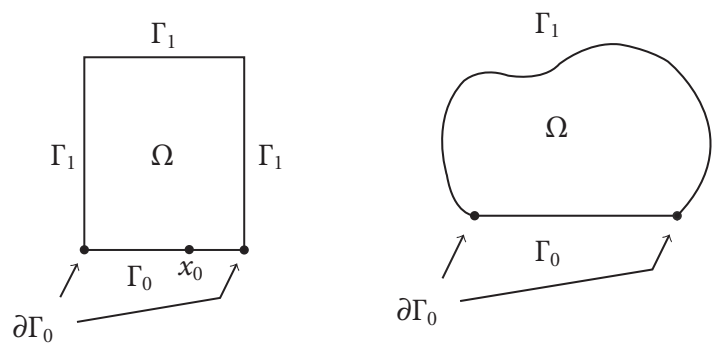

Assumption 1.1. $\mathscr{A}$ (the elastic operator): $L_{2}\left(\Gamma_{0}\right) \supset \mathscr{D}(\mathscr{A}) \rightarrow L_{2}\left(\Gamma_{0}\right)$ is a positive, selfadjoint operator. Moreover, $\rho>0, \beta \geq 0, d_{1}>0, d_{2} \geq 0$, and $1 / 2 \leq \alpha \leq 1$ are constants. Finally, $f \in L_{2}\left(0, T ; L_{2}(\Omega)\right) \equiv L_{2}(Q)$ is an external disturbance.

Assumption 1.2. There exists a constant $r, 0<r<1 / 2$, such that

$$
\mathscr{A}^{-r} \mathscr{B} \in \mathscr{L}\left(\mathscr{U} ; L_{2}\left(\Gamma_{0}\right)\right) ; \text { equivalently, } \mathscr{B} \text { continuous }: \mathscr{U} \longrightarrow\left[\mathscr{D}\left(\mathscr{A}^{r}\right)\right]^{\prime}
$$

Here, $\mathcal{U}$ (control space) is a Hilbert space. Moreover, [ ]'denotes the duality with respect to $L_{2}\left(\Gamma_{0}\right)$ as a pivot space.

Assumption 1.3. $D_{0}: L_{2}\left(\Gamma_{0}\right) \supset \mathscr{D}\left(D_{0}\right) \rightarrow L_{2}\left(\Gamma_{0}\right)$ is a positive, selfadjoint operator, and there exist a constant $r_{0}, 0 \leq r_{0} \leq 1 / 4$, and positive constants $\delta_{1}, \delta_{2}$ such that

$$
\delta_{1}\|z\|_{\mathscr{D}\left(\mathscr{A}^{r_{0}}\right)}^{2} \leq\left(D_{0} z, z\right)_{L_{2}\left(\Gamma_{0}\right)} \leq \delta_{2}\|z\|_{\mathscr{D}\left(\mathscr{A}^{r_{0}}\right)}^{2} \quad \forall z \in \mathscr{D}\left(\mathscr{A}^{r_{0}}\right) \subset \mathscr{D}\left(D_{0}^{1 / 2}\right)
$$

Moreover, it is assumed that $H^{1}\left(\Gamma_{0}\right) \subseteq \mathscr{D}\left(D_{0}^{1 / 2}\right)$, that is,

$$
D_{0}^{1 / 2} \text { : continuous } H^{1}\left(\Gamma_{0}\right) \longrightarrow L_{2}\left(\Gamma_{0}\right) \text {. }
$$

We aim, of course, to achieve the simplest model possible within the above framework, subject to a specific strategy to be enunciated below. In particular, we aim to ascertain if and when we may set $D_{0}=0$ and $\beta=0$, and still achieve both of our sought-after results: singular estimate (1.1) and uniform stabilization of $e^{A t}$. 
Strategy and consequent evolving in the choice of model (1.2). Our viewpoint is as follows. We take as original data of the problem two constants, both associated with the controlled plate $v$-equation (1.2d):

(i) the parameter $0<r<1 / 2$ of (1.3), which measures the degree of unboundness of the control operator $\mathscr{B}$ on the plate;

(ii) the parameter $1 / 2 \leq \alpha \leq 1$ which measures the degree of damping of the structurally damped term $\rho \mathscr{A}^{\alpha} v_{t}, \rho>0$, on the plate (1.2d), within the analyticity range of the free $v$-equation.

Our goal is then to establish the "best" or "minimal" damping $D_{0}$ (constant $r_{0}$ ) that is needed in the boundary conditions (1.2c) of the wave equation on the flexible wall $\Gamma_{0}$, in order to achieve the following three consequential goals on the resulting overall system:

(i) it will be well posed in the semigroup sense over a natural energy space;

(ii) it will satisfy the singular estimate (1.1), with a "best" constant $\gamma$ depending on the data $r$ and $\alpha$ and the minimally necessary constant $r_{0}$, or strength of the damping operator $D_{0}$, in (1.4);

(iii) it will be uniformly stable by introducing, if necessary, an additional boundary term $\beta D_{0} z$ on $\Gamma_{0}, \beta>0$.

As pointed out already in Section 1.1, the analysis of the present paper culminating in Theorem 1.7 shows that there is a conflict between objectives (ii) and (iii), as the damping coefficient $\alpha$ decreases from $\alpha=1$ to $\alpha=1 / 2$, and, say, the degree $r$ of unboundedness of the control operator $\mathscr{B}$ is fixed $(r=3 / 8+\epsilon$ in canonical physical situations). Namely, less plate damping exponent $\alpha$ requires more boundary wave damping $D_{0}$ (or higher $r_{0}$ ) on $\Gamma_{0}$, to the point where $D_{0}$ is of high order: a second-order tangential operator (say LaplaceBeltrami) in order to achieve (1.1) for the physically relevant case $\alpha=1 / 2$. But the high wave damping $D_{0}$ needed to achieve (1.1) causes, in turn, instability of the wave problem (let alone, of the overall problem) when its order is strictly greater than one (overdamping), even with viscous damping $d_{2}>0$ in (1.2a), by forcing the origin $\lambda=0$ to be an element (in fact, the unique element) of the continuous spectrum of the dynamic wave operator (Proposition 4.3) or of the overall system (Remark 4.4). Removing such point in the continuous spectrum when $D_{0}$ is at least a first-order operator, requires, in turn, the insertion of the term $\beta D_{0} z$ on $\Gamma_{0}$, with $\beta>0$ in the wave component, and a renormalization of the state space for $z$, which then preserves dissipativity of the overall new system.

Remark 1.4. Before describing our results, we explicitly note that the phenomenon pointed out above in our present context is generally well known and not surprising, namely, that the insertion of "high" damping in a dynamical system may well work-beyond a certain threshold - as an inhibitor of stability, and even destroy stability (this is referred to as "overdamping"). A simple example is 
the free $v$-problem in $(1.2 \mathrm{~d})$ :

$$
v_{t t}+\mathscr{A} v+\rho \mathscr{A}^{\alpha} v_{t}=0, \quad \rho>0, \alpha \geq \frac{1}{2}
$$

with, say, a positive selfadjoint $\mathscr{A}$ with compact resolvent, as considered in $[6$, Appendix A], from which we quote. There are two branches $\lambda_{n}^{+,-}$of eigenvalues explicitly given in [6, Lemma A.1, page 46]:

(i) for $\alpha>1 / 2$, or $\alpha=1 / 2$ and $\rho \geq 1$, then $\lambda_{n}^{+,-}$are all actually negative for $n$ sufficiently large and $\lambda_{n}^{-} \rightarrow-\infty$ monotonically;

(ii) for $1 / 2<\alpha<1$, or $\alpha=1 / 2$ and $\rho \geq 1$, then $\lambda_{n}^{+} \rightarrow-\infty$ monotonically;

(iii) for $\alpha=1$, then $\lambda_{n}^{+} \nearrow-(1 / 2 \rho)$ monotonically;

(iv) finally, for $\alpha>1$, then $\lambda_{n}^{+} \nearrow 0$ monotonically.

Thus, the spectrum of the dynamic operator corresponding to the free $v$ equation (1.6) (see $A_{2}$ in (2.13) below), is only point spectrum for $\alpha<1$ but contains the point $\lambda=-1 / 2 \rho$ for $\alpha=1$, or the point $\lambda=0$ for $\alpha>1$, in its continuous spectrum. Thus, $A_{2}$ has compact resolvent for $\alpha<1$, but not for $\alpha \geq 1$.

What happens in our wave $z$-component with "high" boundary damping $D_{0}$ is precisely the same phenomenon (see Section 4).

Remark 1.5. Suppose that $\mathscr{A}^{\alpha}$ in $(1.2 \mathrm{~d})$ is replaced with an operator "comparable to it" in the sense of $[5,6,7]$ and [24, Appendix 3B, Chapter 3]. Then, in order to extend the results of this paper to this more general case, one needs to invoke [7, Remark 5.3, page 291] or [24, page 291].

\subsection{Statement of main results: singular estimates with "least" damping $D_{0}$.}

As a preliminary result, we state the following well-posedness result for the uncontrolled model (i.e., $u=0, f=0$ in (1.2)).

THEOREM 1.6 (well-posedness of free problems). With reference to the above setting, assume Hypotheses 1.1 and 1.3 for the coupled $\{z, v\}$-system (1.2). Then, the map $y_{0} \equiv\left[z_{0}, z_{1}, v_{0}, v_{1}\right] \rightarrow\left[z(t), z_{t}(t), v(t), v_{t}(t)\right] \equiv e^{A t} y_{0}$ defines an s.c. contraction semigroup on the state space $Y_{\beta}=Y$ defined in (2.11) below, where the free dynamic operator $A_{\beta}=A$ is identified in (2.37) and (2.38) below.

The above generation result is nonstandard, due to the high damping on $z_{t}$. In particular, it greatly extends [25, Proposition 7.6.2.1, Chapter 7] (see Remark 2.5 and the paragraph below (2.40) for a more technical explanation).

A proof of Theorem 1.6 is given in Appendix A.

Our next main goal is to establish the singular estimate (1.1) with "least" ("best") damping $D_{0}$. To state this result, we define the control operator for the entire structure by $B \equiv[0,0,0, \mathscr{B}]$ (see (2.39) below). In line with our strategy stated above, we will distinguish two cases which yield progressively more complicated models. It turns out that in the range of $\alpha$ near $\alpha=1$ where one may take 
the damping operator $D_{0}$ null: $D_{0}=0$ (and so $\beta=0$ ) in $(1.2 \mathrm{c}$ ), the geometry of the chamber $\Omega$ plays a role (see Orientation at the outset of Section 3.3 below). This is reflected by the parameter $a$ in (1.7b).

Theorem 1.7 (singular estimate). (I) Assume Hypothesis 1.1 (hence $\rho>0 ; \beta \geq 0$; $\left.d_{1}>0, d_{2} \geq 0 ; 1 / 2 \leq \alpha \leq 1\right)$, Hypothesis 1.2 , and, in addition, assume that there exists a constant $\theta_{0}>0$ such that

$$
\begin{aligned}
H^{1-a}\left(\Gamma_{0}\right) & =\mathscr{D}\left(\mathscr{A}^{\theta_{0}}\right), \text { and satisfying } \alpha-2 r \geq 2 \theta_{0}, \\
a & = \begin{cases}\frac{3}{4} & \text { for } \Omega \text { a rectangle }(n=2), \text { or a parallelopiped }(n=3) ; \\
\frac{2}{3} & \text { for } \Omega \text { a general, either smooth or convex, }\end{cases}
\end{aligned}
$$

where we recall that $1 / 2 \leq \alpha \leq 1$ and $0<r<1 / 2$, by Hypotheses 1.1 and 1.2. (We will see in the remarks below that for A a realization of $\Delta^{2}$ as in (1.11), then $\theta_{0}=$ $1 / 16$ for $a=3 / 4$; and $\theta_{0}=1 / 12$ for $a=2 / 3$. Moreover, we refer to Remark 3.7 below for the assertion that the present Part I requires, critically for its validity in the canonical case (1.11), (1.12) for $\mathscr{A}$ and $\mathscr{B}$, that $a>1 / 2$.)

Then, in (1.2d) we can take $D_{0}=0$ and then the corresponding s.c. semigroup $e^{A t}$ on $Y$ guaranteed by Theorem 1.6 and the control operator $B=[0,0,0, \mathscr{B}]$ in (2.39) below satisfy the following singular estimate for some $\omega$ :

$$
\left\|e^{A t} B\right\|_{\mathscr{L}(U ; Y)} \leq C \frac{e^{\omega t}}{t^{\gamma}}, \quad 0<t ; \text { with } \gamma=\frac{r}{\alpha}<\frac{1}{2}
$$

(II) Assume Hypotheses 1.1, 1.2, and 1.3, and, in addition, assume that the parameters $\alpha, r_{0}, r$ of these assumptions satisfy the following constraint:

$$
r_{0}+\frac{\alpha}{2} \geq r \quad \text { or } \quad \alpha-2 r \geq-2 r_{0} .
$$

Then, the singular estimate (1.8) holds true, this time with

$$
\gamma= \begin{cases}\frac{r}{\alpha}, & r \leq \frac{\alpha}{2} \\ \frac{1 / 2-\alpha+r}{1-\alpha}, & r>\frac{\alpha}{2}\end{cases}
$$

where, for $r=\alpha / 2$, both right-hand sides in (1.10) produce the same value $\gamma=1 / 2$. Remarks on, and illustrations of, Theorem 1.7

Remark 1.8 (on canonical realization of $\mathscr{A}$ and $\mathscr{B}$ ). The operators $\mathscr{A}$ and $\mathscr{B}$ which arise in the original canonical structural acoustic model (with $\alpha=1 ; D_{0}=$ 
0 or else $D_{0}=d_{0} I, d_{0}>0 ; \beta=0$ in $\left.(1.2)\right)$ as in $[1,3,16]$, are as follows:

$$
\begin{aligned}
& \mathscr{A}=\text { realization of } \Delta^{2} \text { with appropriate BC (hinged, clamped, free, etc.) } \\
& \mathscr{B} u=\sum_{j=1}^{J} a_{j} u_{j} \delta_{\xi_{j}}^{\prime}, \quad u=\left[u_{1}, \ldots, u_{J}\right] \in \mathbb{R}^{J}=\mathscr{U}(\text { see }[8]),
\end{aligned}
$$

where (i) if $\operatorname{dim} \Gamma_{0}=1(\operatorname{dim} \Omega=2)$, then $\xi_{j}$ are points on $\Gamma_{0}, a_{j}$ are constants, and $\delta_{\xi_{j}}^{\prime}$ are derivatives of the Dirac distribution supported at $\xi_{j}$; while (ii) if $\operatorname{dim} \Gamma_{0}=2(\operatorname{dim} \Omega=3)$, then $\xi_{j}$ denote closed regular curves on $\Gamma_{0}, a_{j}$ are smooth functions, and each $\delta_{\xi_{j}}^{\prime}$ denotes the normal derivative supported at $\xi_{j}$. Model (1.12) for $\mathscr{B}$ arises when the control action is exercised on $\Gamma_{0}$ via piezoceramic elements [8]. Then for $\mathscr{A}$ and $\mathscr{B}$ as in (1.11), (1.12), one readily shows, on the strength of Sobolev embedding, that the constant $r$ of Assumption 1.2 is (see [3], [25, page 890$])$ :

$$
r=\frac{3}{8}+\epsilon, \quad \forall \epsilon>0
$$

Remark 1.9 (on the application of Theorem 1.7(I)). In the canonical case of physical interest where $\mathscr{A}$ is given by (1.11), one always has (see [23, Appendix 3A, Chapter 3, page 284], [10]),

$$
\begin{aligned}
& H^{1 / 4}\left(\Gamma_{0}\right)=\mathscr{D}\left(\mathscr{A}^{1 / 16}\right), \quad \text { in case } a=\frac{3}{4}, 1-a=\frac{1}{4} \\
& H^{1 / 3}\left(\Gamma_{0}\right)=\mathscr{D}\left(\mathscr{A}^{1 / 12}\right), \quad \text { in case } a=\frac{2}{3}, 1-a=\frac{1}{3},
\end{aligned}
$$

under all BC (hinged, clamped, free). Thus, assumption (1.7a) is fulfilled with

$$
\begin{aligned}
& \theta_{0}=\frac{1}{16}, \quad \text { for } a=\frac{3}{4}(\Omega \text { a parallelopiped }) \\
& \theta_{0}=\frac{1}{12}, \quad \text { for } a=\frac{2}{3}(\text { general } \Omega) .
\end{aligned}
$$

Thus, Theorem 1.7(I) applies with $\theta_{0}=1 / 16$ and $\theta_{0}=1 / 12$, respectively, and so, whenever

$$
\begin{array}{ll}
\alpha-2 r \geq \frac{1}{8} & \text { for } a=\frac{3}{4} \\
\alpha-2 r \geq \frac{1}{6} & \text { for } a=\frac{2}{3}
\end{array}
$$


by (1.7a). With $r=3 / 8+\epsilon$ as in the case of $\mathscr{A}$ and $\mathscr{B}$ in (1.11) and (1.12) (see Remark 1.8), this means that Theorem 1.7(I) applies in the range

$$
\begin{aligned}
& \frac{7}{8}<\alpha \leq 1, \quad \text { for } a=\frac{3}{4}(\Omega \text { a parallelopiped }) \\
& \frac{11}{12}<\alpha \leq 1, \quad \text { for } a=\frac{2}{3}(\text { general } \Omega),
\end{aligned}
$$

in which case we can take $r_{0}=0$ and $D_{0}=0$ in (1.2c).

In the case $\alpha=1$ (Kelvin-Voight damping), we obtain $\gamma=r=3 / 8+\epsilon$ in the singular estimate (1.8). We thus recover the result of $[1,3,16]$.

Remark 1.10 (on the application of Theorem 1.7(II)). If $r-\alpha / 2 \leq 0$ or $\alpha \geq 2 r$, we can then take $r_{0}=0$ in (1.9), or $D_{0}$ as a bounded operator on $L_{2}\left(\Gamma_{0}\right)$, say $D_{0}=d_{0} I$ with $d_{0} \geq 0$ by (1.4) and (1.5). In particular, this occurs in the range $1 \geq \alpha>3 / 4$ in the case of $\mathscr{A}$ and $\mathscr{B}$ given by (1.11) and (1.12).

Remark 1.11 (case $\alpha=1 / 2$ with $\mathscr{A}$ and $\mathscr{B}$ given by (1.11) and (1.12)). On the other hand, if $\alpha=1 / 2$, the best $r_{0}$ we may take in (1.9) is $r_{0}=r-\alpha / 2=r-1 / 4$. In the case of $\mathscr{A}$ and $\mathscr{B}$ given by (1.11) and (1.12), where $r=3 / 8+\epsilon$, this means $1 / 8<r_{0} \leq 1 / 4$, according to (1.4) and (1.5). From here we then conclude that $\mathscr{D}\left(\mathscr{A}^{r_{0}}\right)=H_{0}^{4 r_{0}}\left(\Gamma_{0}\right)$ in the case of hinged and clamped BC, and, instead, $\mathscr{D}\left(\mathscr{A}^{r_{0}}\right)=$ $H^{4 r_{0}}\left(\Gamma_{0}\right)$ in the case of free BC for the operator $\mathscr{A}$ as in (1.11) (see [10], [23, page 284]).

Worst subcase $4 r_{0}=1$. Thus, in the worst case $4 r_{0}=1$, we must have $\mathscr{D}\left(D_{0}^{1 / 2}\right) \supset$ $\mathscr{D}\left(\mathscr{A}^{r_{0}}\right)$, where $\mathscr{D}\left(\mathscr{A}^{r_{0}}\right)$ is topologically equivalent to $H^{1}\left(\Gamma_{0}\right)$ in order to satisfy assumption (1.4) and (1.5). Thus, assumption (1.4) and (1.5) on $D_{0}^{1 / 2}$ is fulfilled and $D_{0}$ may be, in this case, the Laplace operator on $\Gamma_{0}$ (second order).

"Best" subcase $4 r_{0}=1 / 2+4 \epsilon$, or $r_{0}-1 / 8+\epsilon, \epsilon>0$. In this case, in order to satisfy assumption (1.4), we must have $\mathscr{D}\left(\mathscr{A}^{r_{0}}\right) \equiv \mathscr{D}\left(\mathscr{A}^{1 / 8+e}\right) \subset \mathscr{D}\left(D_{0}^{1 / 2}\right)$, with $\mathscr{D}\left(\mathscr{A}^{1 / 8+\epsilon}\right)$ topologically equivalent to $H^{1 / 2+4 \epsilon}\left(\Gamma_{0}\right)$. It follows that $D_{0}$ must be of order $s>1$.

Remark 1.12 (range of $\alpha$ for $D_{0}$ a first-order operator and $\mathscr{A}$ and $\mathscr{B}$ given by (1.11) and (1.12)). In this case $\mathscr{D}\left(D_{0}^{1 / 2}\right) \equiv H^{1 / 2}\left(\Gamma_{0}\right)$ and assumption (1.5) is fulfilled. To satisfy assumption (1.4), $\mathscr{D}\left(\mathscr{A}^{r_{0}}\right) \subset \mathscr{D}\left(D_{0}^{1 / 2}\right) \equiv H^{1 / 2}\left(\Gamma_{0}\right)$ with $\mathscr{A}$ given by (1.11), we must take $r_{0}=1 / 8$. In fact, this way, $\mathscr{D}\left(\mathscr{A}^{r_{0}}\right) \equiv \mathscr{D}\left(\mathscr{A}^{1 / 8}\right) \equiv H_{00}^{1 / 2}\left(\Gamma_{0}\right) \subset$ $H^{1 / 2}\left(\Gamma_{0}\right)$ for the case of clamped or hinged BC [36]; and $\mathscr{D}\left(\mathscr{A}^{r_{0}}\right)=\mathscr{D}\left(\mathscr{A}^{1 / 8}\right)=$ $H^{1 / 2}\left(\Gamma_{0}\right)$ in the case of free BC [10], [24, page 284]. Finally, with $r_{0}=1 / 8$ and $\mathscr{B}$ satisfying (1.13), Theorem 1.7 (II) requires $\alpha / 2 \geq r-r_{0}=3 / 8+\epsilon-1 / 8$, or $\alpha \geq$ $1 / 2+2 \epsilon, \epsilon>0$. Thus, the important case $\alpha=1 / 2$ is excluded when $D_{0}$ is a firstorder operator $\mathscr{A}$ and $\mathscr{B}$ are given by (1.11) and (1.12).

As Theorems 1.13 and 1.14 will show, the case where "the operator $D_{0}$ is of order 1 " is the limit case, up to which we may take $\beta=0$ in (1.2c) and still 
obtain uniform (exponential) stability of the free dynamics $e^{A t}$ guaranteed by Theorem 1.6.

Illustration of Theorem 1.7 for $\mathscr{A}$ and $\mathscr{B}$ as in (1.11) and (1.12). (I) For $\mathscr{A}$ as in (1.11) implies

$$
\begin{aligned}
& \theta_{0}=\frac{1}{16}, \quad \text { for } a=\frac{3}{4} \\
& \theta_{0}=\frac{1}{12}, \quad \text { for } a=\frac{2}{3}
\end{aligned}
$$

Can take $D_{0}=0$ for

$$
\begin{aligned}
& \alpha \geq 2 r+\frac{1}{8}, \quad \text { for } a=\frac{3}{4} \\
& \alpha \geq 2 r+\frac{1}{6}, \quad \text { for } a=\frac{2}{3} .
\end{aligned}
$$

Can take $D_{0}=I$ for

$$
2 r \leq \alpha< \begin{cases}2 r+\frac{1}{8}, & \text { for } a=\frac{3}{4} \\ 2 r+\frac{1}{6}, & \text { for } a=\frac{2}{3}\end{cases}
$$

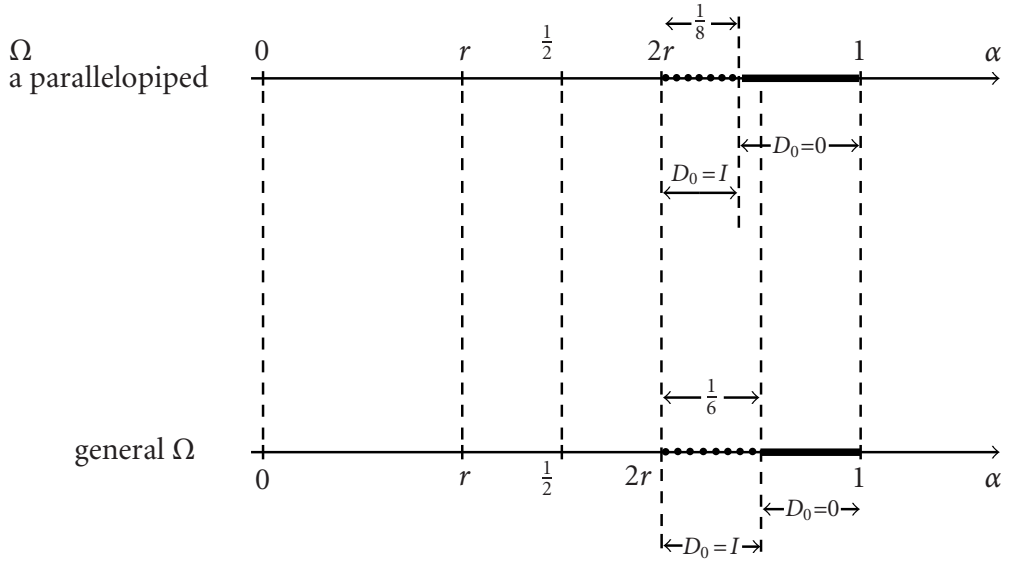

For $\mathscr{B}$ as in (1.12): $r=3 / 8+\epsilon$ :

(II) Can take $D_{0}=$ first-order operator for $1 / 2<\alpha \leq 1$; we have $r_{0}=1 / 8$. Must take $D_{0}$ of order $s, s>1$, to include $\alpha=1 / 2$.

Comparison with the literature. As repeatedly stated, this paper is a de facto successor of the original work on this topic of hyperbolic/parabolic coupled PDE system $[1,3,16]$ which was motivated by the structural acoustic problem with $\mathscr{A}$ 

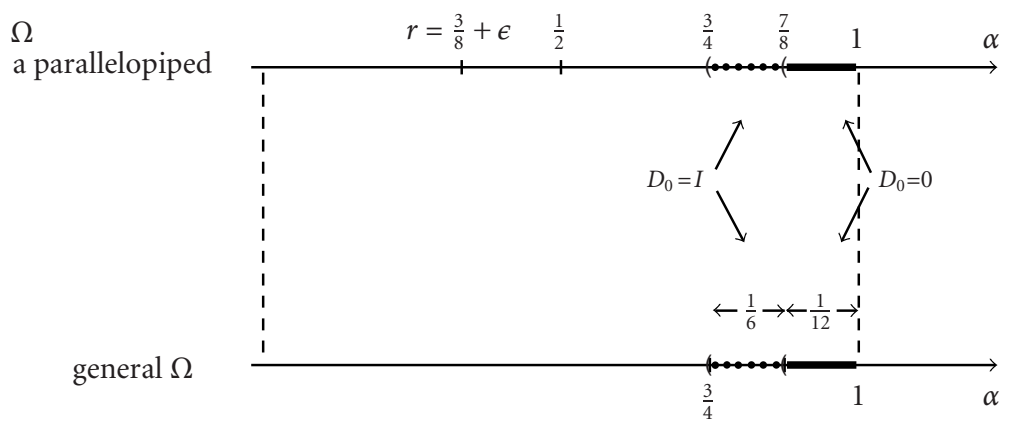

and $\mathscr{B}$ as in (1.11) and (1.12). This work deals with a fixed hyperbolic/parabolic model, contained in (1.2) with $\alpha=1, D_{0}=0$ (or $D_{0}=d_{0} I, d_{0}>0$ ), $\beta=0$ and makes the fundamental discovery that, in this canonical case, the singular estimate (1.8) holds true with $\gamma=r, r$ being the constant in (1.3). In this case $\alpha=1$, viscous damping $d_{2}>0$ readily yields uniform stabilization.

In seeking to establish the singular estimate (1.8) for a "best" coefficient $\gamma$ throughout the entire analyticity range $1 / 2 \leq \alpha \leq 1$, the present paper is confronted not only with additional technicalities, but also with a new strategic paradigm: that of producing the "simplest" variation of the original model for $\alpha=1$, $D_{0}=0, \beta=0$, as to accomplish both of the sought-after goals: (i) the validity of the singular estimate (1.8), and (ii) uniform stability of the overall system which now, unlike the case of $[1,3,16]$, may fail to hold true. This way one can extend the quadratic optimal control theory and related Riccati equations over both a finite and an infinite time horizon [24]. In short, the present paper deals also with an evolving model, where the suitable selection of the "simplest" model at hand responds to the original data $0<r<1 / 2,1 / 2 \leq \alpha \leq 1$ in order to achieve both desired goals. This way, the original model for $\alpha=1, D_{0}=0, \beta=0$ in $[1,3,16]$ has to give way to a more complicated model as $\alpha$ decreases to $1 / 2$, due to the conflict imposed by the two sought-after goals: lower $\alpha$ (lower damping on the plate) requires higher $r_{0}$ (higher damping on the wave on $\Gamma_{0}$ ), and the latter produces instability of the wave component, hence of the overall system. To remove this, the term $\beta D_{0} z$ with $\beta>0$ has to be inserted on the boundary $\Gamma_{0}$ of the $z$-problem. The necessity of $\beta>0$ is established in Proposition 4.3 (see also figures in Appendix C). Of course, our present singular estimate results recover $[1,3,16]$ for $\alpha=1, D_{0}=0, \beta=0$.

At the technical level, our present effort benefits from some obliged strategies already carried out successfully in $[1,3]$, with progressive streamlining and simplification in [16]. As it stands in these references, the proof given there uses the following key ingredients:

(i) analyticity of the s.c. semigroup $e^{A_{2} t}$ describing the dynamics of the flexible wall as established in $[5,6]$ where the generator $A_{2}$ is defined in (2.13) below; 
(ii) characterization of the domains of fractional powers $\left(-A_{2}\right)^{s}$ in the range $0 \leq s \leq 1 / 2$, as established in [7];

(iii) a sharp trace theorem giving, in the case $D_{0}=0$, critical regularity of a second-order hyperbolic equation with Neumann boundary datum; in particular, the sharp trace theory $[2,21,22,23,34]$ of its velocity component;

(iv) decoupling of the $z$-problem and $v$-problem based on the inversion of an operator $\left[I+K_{0}\right]$ on the space $L_{2}\left(0, T ; \mathscr{D}\left(-A_{2}\right)^{\eta}\right), \eta=1 / 2-r$, where $K_{0}$ is a double-integrator operator (cf. [3, equation (46), page 705; equation (65), page 709]). This is done by applying a contraction fixed point first over a sufficiently small terminal interval $[T-\delta, T]$ to get the contraction constant strictly less than one, as required, and independent of the step size $\delta>0$; and then reiterating a finite number of steps to get bounded inversion on all of $[0, T]$.

The first three steps (i), (ii), and (iii) are "obliged" for obtaining the singular estimate (1.8), and we likewise follow this path, with additional technical difficulties embodied by the new Lemma 3.3, and by the use of the characterization of domains of fractional powers $\mathscr{D}\left(\left(-A_{2}\right)^{s}\right)$ this time on the entire range $0 \leq s \leq 1$, and in the dual version, as in Lemma 2.7 below. However, even in the case $\alpha=1 ; D_{0}=0$ (or $\left.D_{0}=d_{0} I, d_{0}>0\right) ; \beta=0$, there is a notable technical simplification in our treatment over that of $[1,3,16]$, which perhaps is responsible for our succeeding in carrying out the generalization to the case $1 / 2 \leq \alpha<1$ in the first place. And this concerns point (iv) above, that is, the issue of untangling the coupling between the $\left\{z, z_{t}\right\}$-variables and the $\left\{v, v_{t}\right\}$-variables. Instead of the fixed point strategy described in (iv) above, we use a definitely simpler approach which consists in taking estimates (rather than keeping exact expressions) and finally resolving the coupling by virtue of the Gronwall's inequality in various forms (see (3.20), (B.9), or (B.15) below).

\subsection{Statement of main results: spectral properties and stability for problem} (1.2) with $f \equiv 0, u \equiv 0$. As described in Section 1.2, the present paper studies also the stability properties of problem (1.2), which are critical in the study of the optimal control problem with quadratic cost functional, over an infinite time horizon. In this respect, our main result is the following.

Theorem 1.13. Assume Hypothesis 1.1 this time with $d_{2}>0$, as well as Hypothesis 1.3. More precisely, under these assumptions, consider the following two cases.

Case 1. Let the operator $D_{0}$ be of order up to 1 ; in particular, let $D_{0}^{1 / 2}:$ continuous $H^{1 / 2}\left(\Gamma_{0}\right) \rightarrow L_{2}\left(\Gamma_{0}\right)$. Then, in this case, take $\beta=0$ in $(1.2 c)$, and hence $Z_{\beta=0} \equiv$ $\mathscr{D}\left(A_{N}^{1 / 2}\right)\left(\right.$ see (2.8)-(2.9) below) and $Y_{\beta=0} \equiv \mathscr{D}\left(A_{N}^{1 / 2}\right) \times L_{2}(\Omega) \times \mathscr{D}\left(\mathscr{A}^{1 / 2}\right) \times L_{2}\left(\Gamma_{0}\right)$ (see (2.11) below).

Case 2. Let now the operator $D_{0}$ be of order $s, 1<s \leq 2$. Then, in this case, take $\beta>0$ in (1.2c) and thus $Y_{\beta} \equiv Z_{\beta} \times L_{2}(\Omega) \times \mathscr{D}\left(\mathscr{A}^{1 / 2}\right) \times L_{2}\left(\Gamma_{0}\right)$ (see (2.11) below). 
Denote now by $A_{\beta}$ the operator (identified in (2.37) and (2.38) below and) called simply A in Theorem 1.6, to emphasize the present role of $\beta$.

Then, in either case, the corresponding s.c. contraction semigroup $e^{A_{\beta} t}$ guaranteed by Theorem 1.6 is uniformly (exponentially) stable on $Y_{\beta}$ : there exists constants $M_{\beta} \geq 1, \omega_{\beta}>0$ (possibly depending on $\beta$ ) such that

$$
\left\|e^{A_{\beta} t}\right\|_{\mathscr{L}\left(Y_{\beta}\right)} \leq M_{\beta} e^{-\omega_{\beta} t}, \quad t \geq 0 .
$$

We provide two radically different proofs of this result. A first proof, given in Section 5, uses energy methods in $t$ (multipliers). A second proof, given in Section 6, uses the stability characterization in terms of the resolvent operator $[9,13,14,30,33]$; and, indeed, provides the more informative Theorem 1.14 below, along with Section 4 . This examines the spectral properties of the origin $\lambda=0$ in terms of the strength of the boundary operator $D_{0}$, to determine, in particular, when the term $\beta D_{0} z$ is critically needed for stability in $(1.2 \mathrm{c})\left(D_{0}\right.$ is of order $s, 1<s \leq 2$ ), and when is not ( $D_{0}$ is of order up to 1$)$. Precise statements are given in Propositions 4.3 and 4.5.

Theorem 1.14. Consider problem (1.2) with $f \equiv 0$ and $u \equiv 0$ (i.e., problem (5.1)), on the space $Y_{\beta}$ under the assumptions that $d_{1} \geq 0, d_{2}>0,0 \leq \alpha \leq 1, \rho>0$, and (1.4) and (1.5). The case $\alpha=0, D_{0}=0$ is explicitly included. Let $\beta \geq 0$ be fixed.

(i) Given $\epsilon>0$, there exists $C_{\epsilon, \beta}$ such that the resolvent operator $R\left(i \omega, A_{\beta}\right)$ of the operator $A_{\beta}$ in (2.37) and (2.38) over $Y_{\beta} \equiv Z_{\beta} \times L_{2}(\Omega) \times \mathscr{D}\left(\mathscr{A}^{1 / 2}\right) \times L_{2}\left(\Gamma_{0}\right)$ (see (2.8), (2.9), (2.10), and (2.11)), as evaluated in the imaginary axis, satisfies the estimate

$$
\left\|R\left(i \omega, A_{\beta}\right)\right\|_{\mathscr{L}\left(Y_{\beta}\right)} \leq C_{\epsilon, \beta}, \quad \forall \omega \in \mathbb{R} \text { s.t. }|\omega| \geq \epsilon .
$$

In particular, $i \mathbb{R}-\{0\} \in \rho\left(A_{B}\right)$ : the imaginary axis, with the origin removed, belongs to the resolvent set of $A_{B}$.

(ii) Assume that $0 \in \rho\left(A_{B}\right)$, the resolvent set of $A_{B}$, this occurs for both Cases 1 and 2 of Theorem 1.13 (see Proposition 4.3). Then, for each $\beta \geq 0$, we can take $\epsilon=0$ in (1.22), so that

$$
\left\|R\left(i \omega, A_{\beta}\right)\right\|_{\mathscr{L}\left(Y_{\beta}\right)} \leq C_{\beta}, \quad \forall \omega \in \mathbb{R}
$$

Accordingly (see $[9,30,33])$, the s.c. contraction semigroup $e^{A_{\beta} t}$ on $Y_{\beta}$ (see Theorem 1.6) is uniformly (exponentially) stable, that is, it satisfies (1.21). The case $\alpha=0$, $D_{0}=0$ is included.

\section{Abstract setting. Preliminaries}

In this section, we introduce the abstract setup for the coupled system (1.2), along with critical preliminary material which is needed in the proof of the main results. Henceforth, for simplicity of notation, we will normalize the physical constants $c$ and $\rho_{1}$, by setting them equal to $1: c=1, \rho_{1}=1$. 
F. Bucci et al. 183

Operators acting on $\Omega$. (i) Let $A_{N}: L_{2}(\Omega) \supset \mathscr{D}\left(A_{N}\right) \rightarrow L_{2}(\Omega)$ be the strictly positive, selfadjoint operator, with $d_{1}>0$, defined by

$$
\begin{aligned}
A_{N} h & =-\Delta h \\
\mathscr{D}\left(A_{N}\right) & =\left\{h \in H^{2}(\Omega):\left.\frac{\partial}{\partial v} h\right|_{\Gamma_{0}}=0,\left[\frac{\partial}{\partial v} h+d_{1} h\right]_{\Gamma_{1}}=0\right\} .
\end{aligned}
$$

Thus, $A_{N}^{-1} \in \mathscr{L}\left(L_{2}(\Omega)\right)$. This is the reason why we are taking $d_{1}>0$ throughout.

(ii) Let $N_{0}$ be the Neumann map [24, page 195] from $L_{2}(\Gamma)$ to $L_{2}(\Omega)$, defined by

$$
\begin{aligned}
& \psi=N_{0} g \Longleftrightarrow\left\{\Delta \psi=0 \text { in } \Omega ;\left.\frac{\partial \psi}{\partial v}\right|_{\Gamma_{0}}=g, \frac{\partial \psi}{\partial v}+\left.d_{1} \psi\right|_{\Gamma_{1}}=0\right\}, \\
& N_{0} \text { continuous : } L_{2}\left(\Gamma_{0}\right) \longrightarrow H^{3 / 2}(\Omega) \subset \mathscr{D}\left(A_{N}^{3 / 4-\epsilon}\right), \quad \epsilon>0,
\end{aligned}
$$

so that

$$
A_{N}^{3 / 4-\epsilon} N_{0} \text { continuous : } L_{2}\left(\Gamma_{0}\right) \longrightarrow L_{2}(\Omega),
$$

or, more generally,

$$
N_{0} \text { continuous : } H^{s}\left(\Gamma_{0}\right) \longrightarrow H^{s+3 / 2}(\Omega), \quad s \in \mathbb{R} \text {. }
$$

Moreover, by Green's second theorem [24, page 196], the following trace results hold true:

$$
N_{0}^{*} A_{N} h= \begin{cases}\left.h\right|_{\Gamma_{0}} & \text { on } \Gamma_{0}, \\ 0 & \text { on } \Gamma_{1},\end{cases}
$$

where $h \in \mathscr{D}\left(A_{N}\right)$, and the validity of (2.6) may be extended to all $h \in H^{1}(\Omega) \equiv$ $\mathscr{D}\left(A_{N}^{1 / 2}\right)$, as $\mathscr{D}\left(A_{N}^{1 / 2}\right)$ is dense in $\mathscr{D}\left(A_{N}\right)$.

Second-order abstract model. By using the Green operators introduced above, the coupled PDE problem (1.2) can be rewritten as the following abstract secondorder system:

$$
\begin{gathered}
z_{t t}+A_{N} z+\beta A_{N} N_{0} D_{0} N_{0}^{*} A_{N} z+A_{N} N_{0} D_{0} N_{0}^{*} A_{N} z_{t}+d_{2} z_{t}-A_{N} N_{0} v_{t}=f \\
v_{t t}+\mathscr{A} v+\rho \mathscr{A}^{\alpha} v_{t}+N_{0}^{*} A_{N} z_{t}=\mathscr{B} u
\end{gathered}
$$

the first equation to be read on $\left[\mathscr{D}\left(A_{N}\right)\right]^{\prime}$, the latter one on $[\mathscr{D}(\mathscr{A})]^{\prime}$ (see $[24$, 25]).

Function spaces and operators. Next we define the space

$$
Z_{\beta} \equiv\left\{h \in \mathscr{D}\left(A_{N}^{1 / 2}\right) \equiv H^{1}(\Omega):\left.h\right|_{\Gamma_{0}} \in \mathscr{D}\left(D_{0}^{1 / 2}\right)\right\},
$$


endowed with the norm

$$
\|h\|_{Z_{\beta}}^{2} \equiv\left\|A_{N}^{1 / 2} h\right\|_{L_{2}(\Omega)}^{2}+\beta\left\|D_{0}^{1 / 2} N_{0}^{*} A_{N} h\right\|_{L_{2}\left(\Gamma_{0}\right)}^{2},
$$

which is needed to describe the state space of problem (2.7) (or (1.2)). (Notice that, for $\beta=0$, we then have $Z_{\beta}=\mathscr{D}\left(A_{N}^{1 / 2}\right)$.) In fact, the function spaces $Y_{1}$ for the wave component $\left[z, z_{t}\right]$ and $Y_{2}$ for the plate component $\left[v, v_{t}\right]$ of system $(2.7)$ are given, respectively, by

$$
Y_{1, \beta} \equiv Y_{1} \equiv Z_{\beta} \times L_{2}(\Omega) ; \quad Y_{2} \equiv \mathscr{D}\left(\mathscr{A}^{1 / 2}\right) \times L_{2}\left(\Gamma_{0}\right) \text {. }
$$

The state space for problem (2.7) is then

$$
Y_{\beta} \equiv Y \equiv Y_{1} \times Y_{2}=Z_{\beta} \times L_{2}(\Omega) \times \mathscr{D}\left(\mathscr{A}^{1 / 2}\right) \times L_{2}\left(\Gamma_{0}\right) .
$$

Remark 2.1. Note that the operator $D_{0}^{1 / 2} N_{0}^{*} A_{N}$ is a densely defined, closed operator on $\mathscr{D}\left(A_{N}^{1 / 2}\right) \equiv H^{1}(\Omega)$. In fact, for instance, $\mathscr{D}\left(A_{N}\right) \subset \mathscr{D}\left(D_{0}^{1 / 2} N_{0}^{*} A_{N}\right)$, since by Assumption 1.3, $D_{0}^{1 / 2}$ is bounded from $H^{1}\left(\Gamma_{0}\right)$ into $L_{2}\left(\Gamma_{0}\right)$ (see (1.5)). Furthermore, $D_{0}^{1 / 2} N_{0}^{*} A_{N}$ is closed being the composition of the closed boundedly invertible operator $D_{0}^{1 / 2}$ with the operator $N_{0}^{*} A_{N}$ which is bounded on $\mathscr{D}\left(A_{N}^{1 / 2}\right)$ [15, page 164] (see (2.4) and (2.5)).

Remark 2.2. From definitions (2.8) and (2.9) it follows that $Z_{\beta}$ reduces to $\mathscr{D}\left(A_{N}^{1 / 2}\right)$ when $\beta=0$. Also, if $r_{0}=0$ in (1.4) and (1.5), then $D_{0}$ is a bounded operator on $L_{2}\left(\Gamma_{0}\right)$ and therefore the norm defined by (2.9) is equivalent to the usual one in the space $\mathscr{D}\left(A_{N}^{1 / 2}\right)$. More generally, if $D_{0}$ is an operator of order 1 , that is, if $D_{0}^{1 / 2}$ : continuous $H^{1 / 2}\left(\Gamma_{0}\right) \rightarrow L_{2}\left(\Gamma_{0}\right)$, then the norm (2.9) for $Z_{\beta}$ is equivalent to the $\mathscr{D}\left(A_{N}^{1 / 2}\right)$-norm. For $\mathscr{A}$ being the realization of fourth-order elastic operator, as given by (1.11), this is the case for all $r_{0} \in[0,1 / 8]$. In fact, in the worst case, as we have seen in Remark 1.10, $\mathscr{D}\left(\mathscr{A}^{r_{0}}\right)$ is topologically equivalent to $H^{4 r_{0}}\left(\Gamma_{0}\right)=$ $H^{1 / 2}\left(\Gamma_{0}\right)$ for $r_{0}=1 / 8$. We have seen in Remarks 1.11 and 1.12 that then we must have $1 / 2<\alpha \leq 1$.

Accordingly, we define the operators $A_{1}: Y_{1} \supset \mathscr{D}\left(A_{1}\right) \rightarrow Y_{1}$ and $A_{2}: Y_{2} \supset$ $\mathscr{D}\left(A_{2}\right) \rightarrow Y_{2}$ as follows:

$$
\begin{aligned}
& A_{1}=\left[\begin{array}{cc}
0 & I \\
-A_{N}-\beta A_{N} N_{0} D_{0} N_{0}^{*} A_{N} & -A_{N} N_{0} D_{0} N_{0}^{*} A_{N}-d_{2} I
\end{array}\right], \\
& \mathscr{D}\left(A_{1}\right)=\left\{\left[h_{1}, h_{2}\right]: h_{1}, h_{2} \in Z_{\beta}\right. \text {, } \\
& \left.h_{1}+\beta N_{0} D_{0} N_{0}^{*} A_{N} h_{1}+N_{0} D_{0} N_{0}^{*} A_{N} h_{2} \in \mathscr{D}\left(A_{N}\right)\right\} ; \\
& A_{2}=\left[\begin{array}{cc}
0 & I \\
-\mathscr{A} & -\rho \mathscr{A}^{\alpha}
\end{array}\right] \text {, } \\
& \mathscr{D}\left(A_{2}\right)=\left\{\left[h_{1}, h_{2}\right]: h_{1}, h_{2} \in \mathscr{D}\left(\mathscr{A}^{1 / 2}\right): \mathscr{A}^{1-\alpha} h_{1}+\rho h_{2} \in \mathscr{D}\left(\mathscr{A}^{\alpha}\right)\right\} \text {. }
\end{aligned}
$$


The adjoint operators are given, respectively, by

$$
\begin{aligned}
& A_{1}^{*}=\left[\begin{array}{cc}
0 & -I \\
A_{N}+\beta A_{N} N_{0} D_{0} N_{0}^{*} A_{N} & -A_{N} N_{0} D_{0} N_{0}^{*} A_{N}-d_{2} I
\end{array}\right], \\
& A_{2}^{*}=\left[\begin{array}{cc}
0 & -I \\
\mathcal{A} & -\rho \mathscr{A}^{\alpha}
\end{array}\right],
\end{aligned}
$$

with domains analogously defined.

Remark 2.3 (even for $\beta=0$ ). Equation (2.12b) implies that $D_{0}^{1 / 2} N_{0}^{*} A_{N} h_{2} \in L_{2}\left(\Gamma_{0}\right)$ for $\left\{h_{1}, h_{2}\right\} \in \mathscr{D}\left(A_{1}\right)$ (compare with (2.8) and (2.9) for $\beta>0$ ).

In fact, $(2.12 \mathrm{~b})$, for $\beta=0$ and with $\mathscr{D}\left(A_{N}\right) \subset \mathscr{D}\left(A_{N}^{1 / 2}\right)$, implies at first that $N_{0} D_{0} N_{0}^{*} A_{N} h_{2} \in \mathscr{D}\left(A_{N}^{1 / 2}\right)$, since $h_{1} \in \mathscr{D}\left(A_{N}^{1 / 2}\right)$. It then follows that $A_{N} N_{0} D_{0} N_{0}^{*} A_{N} h_{2}$ $\in\left[\mathscr{D}\left(A_{N}^{1 / 2}\right)\right]^{\prime}$, and hence that

$$
\left\|D_{0}^{1 / 2} N_{0}^{*} A_{N} h_{2}\right\|_{L_{2}\left(\Gamma_{0}\right)}^{2}=\left(A_{N} N_{0} D_{0} N_{0}^{*} A_{N} h_{2}, h_{2}\right)_{L_{2}\left(\Gamma_{0}\right)}<\infty,
$$

as $h_{2} \in \mathscr{D}\left(A_{N}^{1 / 2}\right)$ as well.

Generation results for $A_{1}$ and $A_{2}$, and fractional powers of $\left(-A_{2}\right)$. We begin with the statement of the main properties of the operators $A_{1}$ and $A_{2}$ which will be used in the sequel. In particular, the generation properties of $A_{1}$ and $A_{2}$ will eventually lead to the proof of well-posedness of the first-order abstract system to which (2.7) (hence (1.2)) will be reduced.

Lemma 2.4. Assume Hypotheses 1.1, 1.2, and 1.3. With $d_{1}>0$, for all $d_{2}, \beta \geq 0$, the operators $A_{1}$ and $A_{1}^{*}$ defined by (2.12), and (2.14) are maximal dissipative on the space $Y_{1}$ defined by (2.10) and hence are the generators of strongly continuous semigroups $e^{A_{1} t}$ and $e^{A_{1}^{*} t}$ of contractions on $Y_{1}, t \geq 0$.

Proof. This is given in Appendix A and is based on the critical role of the norm (2.9) for the space $Z_{\beta}$.

Remark 2.5. The presence of the high damping term $D_{0} z_{t}$ in $(1.2 \mathrm{c})$, hence of the term $-A_{N} N_{0} D_{0} N_{0}^{*} A_{N}$ in (2.12a) makes the present generation result of Lemma 2.4 nonstandard; in particular, outside the scope of [25, Proposition 7.6.2.1, Chapter 7, page 664].

Remark 2.6 (for $\beta \geq 0$ ). Let $\left\{z_{0}, z_{1}\right\} \in \mathscr{D}\left(A_{1}\right)$. Then, by Lemma 2.4,

$$
\begin{aligned}
\left\{z, z_{t}\right\} & =e^{A_{1} \cdot}\left[z_{0}, z_{1}\right] \in C\left([0, T] ; \mathscr{D}\left(A_{1}\right)\right), \\
\left\{z_{t}, z_{t t}\right\} & =e^{A_{1} \cdot} A_{1}\left[z_{0}, z_{1}\right] \in C\left([0, T] ; Y_{1}\right) .
\end{aligned}
$$

Explicitly, via (2.12b) and (2.10), this implies that

$$
\begin{gathered}
z, z_{t} \in C\left([0, T] ; H^{1}(\Omega)\right), \quad z_{t t} \in C\left([0, T] ; L_{2}(\Omega)\right), \\
\text { hence } \Delta z \in C\left([0, T] ; L_{2}(\Omega)\right) .
\end{gathered}
$$


Moreover, recalling Remark 2.3 for $\beta=0$, and (2.8) and (2.9) for $\beta>0$, we obtain

$$
D_{0}^{1 / 2} N_{0}^{*} A_{N} z_{t}(t) \in C\left([0, T] ; L_{2}\left(\Gamma_{0}\right)\right)
$$

Hence,

$$
\begin{gathered}
D_{0}^{1 / 2} N_{0}^{*} A_{N} z(t)=D_{0}^{1 / 2} N_{0}^{*} A_{N} z(0)+\int_{0}^{t} D_{0}^{1 / 2} N_{0}^{*} A_{N} z_{\tau}(\tau) d \tau \in C^{1}\left([0, T] ; L_{2}\left(\Gamma_{0}\right)\right) \\
\text { if } D_{0}^{1 / 2} N_{0}^{*} A_{N} z(0) \in L_{2}\left(\Gamma_{0}\right) .
\end{gathered}
$$

Finally, for $\beta=0$, let $\mathscr{D}$ be the subspace of $\mathscr{D}\left(A_{1}\right)$ defined by

$$
\mathscr{D} \equiv\left\{\left[z_{1}, z_{2}\right] \in \mathscr{D}\left(A_{1}\right): D_{0}^{1 / 2} N_{0}^{*} A_{N} z_{1} \in L_{2}\left(\Gamma_{0}\right)\right\} .
$$

Then, we have that $\mathscr{D}$ is invariant under the resolvent $R\left(\lambda, A_{1}\right)$ of $A_{1}$; that is,

$$
R\left(\lambda, A_{1}\right) \mathscr{D} \subset \mathscr{D}, \quad \text { for } \beta=0, \operatorname{Re} \lambda>0 .
$$

Indeed, writing $-R\left(\lambda, A_{1}\right)\left[z_{1}, z_{2}\right]=\left[y_{1}, y_{2}\right] \in \mathscr{D}\left(A_{1}\right)$ for $\left[z_{1}, z_{2}\right] \in \mathscr{D}$, by $(2.12 \mathrm{a})$ and (2.12b) with $\beta=0$ and, say, $d_{2}=0$ without loss of generality, we obtain $y_{1}=\left(y_{2}-z_{1}\right) / \lambda$, with $\left[y_{1}, y_{2}\right] \in \mathscr{D}$, as desired, since by Remark 2.3 we have $D_{0}^{1 / 2} N_{0}^{*} A_{N} y_{2} \in L_{2}\left(\Gamma_{0}\right)$.

In the following lemma, we recall from $[5,6,7]$ a set of results concerning the operator $A_{2}$ of paramount importance here.

Lemma 2.7 (see $[5,6,7]$ ). Assume Hypothesis 1.1 on $A$.

(i) For every $\alpha \in[1 / 2,1]$, the operators $A_{2}$ and $A_{2}^{*}$ defined by (2.13) and (2.15) are maximal dissipative on the space $Y_{2}$ defined by (2.10), and hence are the generators of strongly continuous semigroups $e^{A_{2} t}$ and $e^{A_{2}^{*} t}$ of contractions on $Y_{2}, t \geq 0$, which moreover are analytic for $t>0$, as well as (uniformly) exponentially stable on $Y_{2}$ : there exist constants $C \geq 1, a>0$ such that $\left\|e^{A t}\right\|_{\mathscr{L}\left(Y_{1}\right)} \leq C e^{-a t}, t \geq 0$. Also, $A_{2}^{-1} \in \mathscr{L}\left(Y_{2}\right)$.

(ii) For every $s \in[0,1]$, the fractional powers $\left(-A_{2}\right)^{s}$ of $A_{2}$ are well defined and their domains are given as follows:

(a) if $0 \leq s \leq 1 / 2$, then

$$
\begin{gathered}
\mathscr{D}\left(\left(-A_{2}\right)^{s}\right)=\mathscr{D}\left(\mathscr{A}^{1 / 2+s(1-\alpha)}\right) \times \mathscr{D}\left(\mathscr{A}^{\alpha s}\right) ; \\
\left\|\left(-A_{2}\right)^{s}\left[\begin{array}{l}
x_{1} \\
x_{2}
\end{array}\right]\right\|_{Y_{2}}^{2}=\left\|\mathscr{A}^{1 / 2+s(1-\alpha)} x_{1}\right\|_{L_{2}\left(\Gamma_{0}\right)}^{2}+\left\|\mathscr{A}^{\alpha s} x_{2}\right\|_{L_{2}\left(\Gamma_{0}\right)}^{2} ;
\end{gathered}
$$

(b) if $1 / 2 \leq s \leq 1$, then

$$
\begin{aligned}
\mathscr{D}\left(\left(-A_{2}\right)^{s}\right)= & \left\{[x, y]: x \in D\left(\mathscr{A}^{1 / 2+s(1-\alpha)}\right),\right. \\
& \left.y \in \mathscr{D}\left(\mathscr{A}^{\alpha-1 / 2+s(1-\alpha)}\right) ; \mathscr{A}^{1-\alpha} x+\rho y \in \mathscr{D}\left(\mathscr{A}^{\alpha s}\right)\right\} .
\end{aligned}
$$


The previous characterization of domains of fractional powers $\left(-A_{2}\right)^{s}$ of $-A_{2}$ allows us to establish some properties of their inverse, along with their corresponding norms, which will be critically used in the proof of Theorem 1.7.

Lemma 2.8. Assume Hypothesis 1.1 on $\mathscr{A}$.

(i) If $0 \leq s \leq 1 / 2$, then

$$
\begin{aligned}
\left\|A_{2}^{-s}\left[\begin{array}{l}
v_{1} \\
v_{2}
\end{array}\right]\right\|_{Y_{2}}^{2}=\left\|\mathscr{A}^{1 / 2-s(1-\alpha)} v_{1}\right\|_{L_{2}\left(\Gamma_{0}\right)}^{2}+ & \left\|\mathscr{A}^{-\alpha s} v_{2}\right\|_{L_{2}\left(\Gamma_{0}\right)}^{2}, \\
& {\left[\begin{array}{l}
v_{1} \\
v_{2}
\end{array}\right] }
\end{aligned}
$$

so that in particular,

$$
\left\|A_{2}^{-s}\left[\begin{array}{c}
0 \\
v_{2}
\end{array}\right]\right\|_{Y_{2}}^{2}=\left\|\mathscr{A}^{-\alpha s} v_{2}\right\|_{L_{2}\left(\Gamma_{0}\right)}^{2}, \quad v_{2} \in\left[\mathscr{D}\left(\mathscr{A}^{\alpha s}\right)\right]^{\prime}
$$

In (2.25), [ ]' (resp., ( )') denotes duality with respect to $Y_{2}$ (resp., $L_{2}\left(\Gamma_{0}\right)$ ) as a pivot space.

(ii) If, instead, $1 / 2 \leq s \leq 1$, then

$$
\begin{array}{r}
\left\|A_{2}^{-s}\left[\begin{array}{l}
v_{1} \\
v_{2}
\end{array}\right]\right\|_{Y_{2}}^{2} \leq k\left(\left\|\mathscr{A}^{1 / 2-s(1-\alpha)} v_{1}\right\|_{L_{2}\left(\Gamma_{0}\right)}^{2}+\left\|\mathscr{A}^{1 / 2-\alpha-s(1-\alpha)} v_{2}\right\|_{L_{2}\left(\Gamma_{0}\right)}^{2}\right), \\
{\left[\begin{array}{l}
v_{1} \\
v_{2}
\end{array}\right] \in\left[\mathscr{D}\left(\left(-A_{2}\right)^{s}\right)\right]^{\prime},}
\end{array}
$$

for some constant $k$, so that in particular,

$$
\left\|A_{2}^{-s}\left[\begin{array}{c}
0 \\
v_{2}
\end{array}\right]\right\|_{Y_{2}}^{2} \leq k\left\|\mathscr{A}^{1 / 2-\alpha-s(1-\alpha)} v_{2}\right\|_{L_{2}\left(\Gamma_{0}\right)}^{2}, \quad v_{2} \in\left(\mathscr{D}\left(\mathscr{A}^{\alpha-1 / 2+s(1-\alpha)}\right)\right)^{\prime} .
$$

Proof of Lemma 2.8. (i) Let $0 \leq s \leq 1 / 2$. Then, from the first characterization (2.23) it follows that

$$
\left[\mathscr{D}\left(\left(-A_{2}\right)^{s}\right)\right]^{\prime}=\mathscr{D}\left(\mathscr{A}^{1 / 2-s(1-\alpha)}\right) \times\left(D\left(\mathscr{A}^{\alpha s}\right)\right)^{\prime},
$$

where [ ]' in the left-hand side of (2.29) denotes duality with respect to $Y_{2}$ as a pivot space, while $(\quad)^{\prime}$ on the right-hand side denotes duality with respect to $L_{2}\left(\Gamma_{0}\right)$, respectively, as a pivot space. Hence, (2.29) implies (2.25), which yields estimate (2.26) as a special case.

(ii) Let now $1 / 2 \leq s \leq 1$. Characterization (2.24) implies, a fortiori,

$$
\mathscr{D}\left(\left(-A_{2}\right)^{s}\right) \subseteq \mathscr{D}\left(\mathscr{A}^{1 / 2+s(1-\alpha)}\right) \times \mathscr{D}\left(\mathscr{A}^{\alpha-1 / 2+s(1-\alpha)}\right),
$$


it then follows that

$$
\mathscr{D}\left(\mathscr{A}^{1 / 2-s(1-\alpha)}\right) \times\left(\mathscr{D}\left(\mathscr{A}^{\alpha-1 / 2+s(1-\alpha)}\right)\right)^{\prime} \subseteq\left[\mathscr{D}\left(\left(-A_{2}\right)^{s}\right)\right]^{\prime},
$$

with continuous injection, and dualities to be understood as before. Therefore, (2.31) implies estimate (2.27), and this yields estimate (2.28), again as a special case.

Coupling. Finally, we introduce the densely defined (unbounded, unclosable) trace operator $C: Y_{1} \supset \mathscr{D}(C) \rightarrow Y_{2}$ defined by

$$
C\left[\begin{array}{l}
z_{1} \\
z_{2}
\end{array}\right] \equiv\left[\begin{array}{c}
0 \\
N_{0}^{*} A_{N} z_{2}
\end{array}\right]=\left[\begin{array}{cc}
0 & 0 \\
0 & N_{0}^{*} A_{N}
\end{array}\right]\left[\begin{array}{l}
z_{1} \\
z_{2}
\end{array}\right],
$$

with domain (see (2.4) and (2.5))

$$
\begin{aligned}
\mathscr{D}(C) & =\left\{\left[z_{1}, z_{2}\right] \in Y_{1}: N_{0}^{*} A_{N} z_{2}=\left.z_{2}\right|_{\Gamma_{0}} \in L_{2}\left(\Gamma_{0}\right)\right\} \\
& \supset \mathscr{D}\left(A_{N}^{1 / 2}\right) \times D\left(A_{N}^{1 / 4+\epsilon}\right), \quad \epsilon>0,
\end{aligned}
$$

so that $\mathscr{D}\left(A_{N}^{1 / 2}\right) \times \mathscr{D}\left(A_{N}^{1 / 2}\right) \subset \mathscr{D}(C)$. Its adjoint

$$
C^{*}: Y_{2} \longrightarrow \mathscr{D}\left(A_{N}^{1 / 2}\right) \times\left[\mathscr{D}\left(A_{N}^{1 / 4+\epsilon}\right)\right]^{\prime},
$$

in the sense that $\left(C y_{1}, y_{2}\right)_{Y_{2}}=\left(y_{1}, C^{*} y_{2}\right)_{Y_{1}}$, is given by

$$
C^{*}\left[\begin{array}{l}
v_{1} \\
v_{2}
\end{array}\right]=\left[\begin{array}{c}
0 \\
A_{N} N_{0} v_{2}
\end{array}\right]=\left[\begin{array}{cc}
0 & 0 \\
0 & A_{N} N_{0}
\end{array}\right]\left[\begin{array}{l}
z_{1} \\
z_{2}
\end{array}\right],
$$

where $A_{N} N_{0}: L_{2}\left(\Gamma_{0}\right) \rightarrow\left[\mathscr{D}\left(A_{N}^{1 / 4+\epsilon}\right)\right]^{\prime}$, recalling properties (2.4) and (2.5).

First-order abstract model. Dynamics operator. Finally, from (2.12), (2.13), (2.32), and (2.35), we define the operator

$$
\begin{aligned}
A & =\left[\begin{array}{ll}
A_{1} & C^{*} \\
-C & A_{2}
\end{array}\right]: Y \supset \mathscr{D}(A) \longrightarrow Y \\
& =Y_{1} \times Y_{2}=Z_{\beta} \times L_{2}(\Omega) \times \mathscr{D}\left(\mathscr{A}^{1 / 2}\right) \times L_{2}\left(\Gamma_{0}\right),
\end{aligned}
$$

which explicitly reads as follows:

$$
\begin{aligned}
& A_{\beta} \equiv A
\end{aligned}
$$

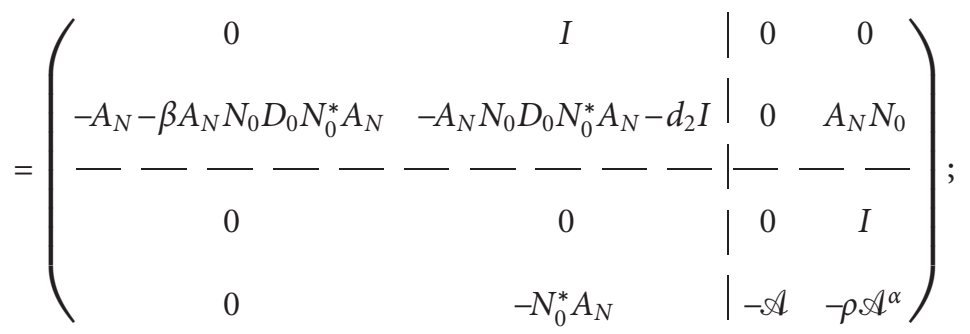


with domain

$$
\begin{array}{r}
\mathscr{D}(A)=\left\{\left[z_{1}, z_{2}, v_{1}, v_{2}\right] \in Y: z_{2} \in Z_{\beta}, v_{2} \in \mathscr{D}\left(\mathscr{A}^{1 / 2}\right), \mathscr{A}^{1-\alpha} v_{1}+\rho v_{2} \in \mathscr{D}\left(\mathscr{A}^{\alpha}\right),\right. \\
\left.z_{1}+\beta N_{0} D_{0} N_{0}^{*} A_{N} z_{1}+N_{0} D_{0} N_{0}^{*} A_{N} z_{2}-N_{0} v_{2} \in \mathscr{D}\left(A_{N}\right)\right\} .
\end{array}
$$

Control operator. Finally, we set $U=0 \times 0 \times 0 \times \mathcal{u}$, set $\tilde{u}=[0,0,0, u], u \in \mathcal{u}$, and define the operator $B: U \rightarrow\left[\mathscr{D}\left(A^{*}\right)\right]^{\prime}$, duality with respect to $Y$, as a pivot space, as well as $F$ by

$$
B \tilde{u}=\left[\begin{array}{c}
0 \\
0 \\
0 \\
\mathscr{B} u
\end{array}\right] ; \quad F=\left[\begin{array}{l}
0 \\
f \\
0 \\
0
\end{array}\right] ; \quad A^{-1} B=\left[\begin{array}{c}
0 \\
0 \\
-\mathscr{A}^{-1} \mathscr{B} \\
0
\end{array}\right] \in \mathscr{L}(U ; Y),
$$

so that $B \in \mathscr{L}\left(U ;\left[\mathscr{D}\left(A^{*}\right)\right]^{\prime}\right)$. Here we have used $(1.3)$ to deduce that $\mathscr{A}^{-1} \mathscr{B}=$ $\mathscr{A}^{r-1} \mathscr{A}^{-r} \mathscr{B}$ is bounded from $\mathcal{U}$ into $L_{2}\left(\Gamma_{0}\right)$, where $r<1 / 2$. To obtain the form of $A^{-1} B$, we write explicitly the system $A\left[z_{1}, z_{2}, v_{1}, v_{2}\right]=B \tilde{u}$ by using (2.37), (2.38), and (2.39) and readily obtain $z_{2}=0, v_{2}=0,\left\|A_{N}^{1 / 2} z_{1}\right\|^{2}+\beta\left\|D_{0}^{1 / 2} N_{0}^{*} A_{N} z_{1}\right\|^{2}=0$, hence $z_{1}=0$; then $-\mathscr{A} v_{1}=\mathscr{B} u$ which finally yields $v_{1}=-\mathscr{A}^{-1} \mathscr{B} u$.

Finally, returning to the second-order abstract model (2.7), we see that these equations can be rewritten as the following first-order abstract equation in the variable $y(t)=\left[z(t), z_{t}(t), v(t), v_{t}(t)\right]$ :

$$
y^{\prime}=A y+B \tilde{u}+F \quad \text { in }\left[\mathscr{D}\left(A^{*}\right)\right]^{\prime},
$$

where $A, B, F$ are defined in (2.37), (2.38), and (2.39), respectively.

Theorem 1.6 claims well-posedness of (2.40) with $\tilde{u} \equiv 0, F \equiv 0$, in the sense that the operator $A$ in (2.37) and (2.38) is maximal dissipative and hence generates an s.c. semigroup of contractions $e^{A t}$ on the space $Y$ defined by (2.11). As noted in Remark 2.5, Theorem 1.6 is nonstandard due to the high damping term $D_{0} z_{t}$ in (1.2c), hence of the term $-A_{N} N_{0} D_{0} N_{0}^{*} A_{N}$ in (2.37) acting on the second coordinate. In particular, we cannot invoke [25, Proposition 7.6.2.1, Chapter 7, page 664].

\section{Proof of Theorem 1.7: singular estimate for $e^{A t} B$}

The goal of this section is to establish the singular estimate (1.8) under the conditions of Theorem 1.7(I), (II).

3.1. Orientation. We have already observed that the s.c. semigroup $e^{A t}$, originally defined on the space $Y$ in (2.11), or (2.36), as guaranteed by Theorem 1.6, can, in fact, be extended as an s.c. semigroup on $\left[\mathscr{D}\left(A^{*}\right)\right]^{\prime}$ as well, where [ ]' denotes duality with respect to $Y$ as a pivot space. Thus, according to property (2.39) for $B$, we have that $y(t) \equiv e^{A t} B \tilde{u} \in C\left([0, T] ;\left[\mathscr{D}\left(A^{*}\right)\right]^{\prime}\right)$ and satisfies 
$\dot{y}=A y \in\left[\mathscr{D}\left(A^{*}\right)\right]^{\prime}, y(0)=B \tilde{u}$ for all $\tilde{u} \in U$. Our goal is to drastically improve upon this regularity result and show that, in fact, $e^{A t} B \tilde{u} \in C((0, T] ; Y)$ and, moreover, it satisfies the singular estimate (1.8) as $t \downarrow 0$.

However, the original solution $y(t)=e^{A t} B \tilde{u}$ is not sufficiently regular in space to justify the required computations in the arguments below. This fact then requires that we start with another, smoother initial condition $y(0) \in \mathscr{D}(A)$ so that, in view of Theorem 1.6, the corresponding solution $y(t)=e^{A t} y(0) \in C([0, T]$; $\mathscr{D}(A))$ possesses the required regularity properties in space. Due to the form of $B \tilde{u}=[0,0,0, \mathscr{B} u]$ in (2.39), where $\mathscr{B} u \in\left[\mathscr{D}\left(\mathscr{A}^{r}\right)\right]^{\prime}$ or $\mathscr{A}^{-r} \mathscr{B} u \in L_{2}\left(\Gamma_{0}\right)$, by $(1.3)$, a natural first guess would be to take an initial condition of the type $\left[0,0,0, v_{2}\right]$ with $0 \neq v_{2}$ sufficiently smooth, but to be penalized on the final estimates in the $\left[\mathscr{D}\left(\mathscr{A}^{r}\right)\right]^{\prime}$-norm; so that, in the end, by continuity, $v_{2}$ can be substituted with $\mathscr{B} u$ in the final estimates. However, the problem with this choice is that no matter how smooth $v_{2}$ is, the point $\left[0,0,0, v_{2}\right]$ can never belong to $\mathscr{D}(A)$, as $(2.38)$ readily reveals. Thus, we modify our initial guess and take, instead, a sequence of initial conditions $y_{0, n}=\left[z_{0, n}, 0,0, v_{2}\right] \in \mathscr{D}(A)$ - this is now possible by (2.38)! penalize $v_{2}$ in the $\left[\mathscr{D}\left(\mathscr{A}^{r}\right)\right]^{\prime}$-norm and, at the end, let $z_{0, n} \rightarrow 0$, in order to recover the desired initial condition $[0,0,0, \mathscr{B} u]$ by continuity. This is what we will do next.

Thus, as explained above, we will begin by analyzing the following first-order problem:

$$
\dot{y}=A y, \quad y(0) \in \mathscr{D}(A)
$$

where $A$ is given by (2.37) and (2.38); or, more specifically, its corresponding second-order version

$$
\begin{gathered}
z_{t t}+A_{N} z+\beta A_{N} N_{0} D_{0} N_{0}^{*} A_{N} z+A_{N} N_{0} D_{0} N_{0}^{*} A_{N} z_{t}+d_{2} z_{t}-A_{N} N_{0} v_{t}=0, \\
v_{t t}+\mathscr{A} v+\rho \mathscr{A}^{\alpha} v_{t}+N_{0}^{*} A_{N} z_{t}=0, \\
y(0)=\left\{z(0)=z_{0}, z_{t}(0)=0, v(0)=0, v_{t}(0)=v_{2}\right\} \in \mathscr{D}(A),
\end{gathered}
$$

which is system (2.7) (i.e., problem (1.2)) with $f \equiv 0, u \equiv 0$, and a special initial condition. Then, according to Theorem 1.6, the solution of problem (3.2) satisfies the regularity property

$$
y(t)=\left[z(t), z_{t}(t), v(t), v_{t}(t)\right]=e^{A t} y(0) \in C([0, T] ; \mathscr{D}(A)),
$$

that is, a fortiori, from (2.38),

$$
\begin{aligned}
& y_{1}(t)=\left[z(t), z_{t}(t)\right] \in C\left([0, T] ; Z_{\beta} \times Z_{\beta}\right) \\
& y_{2}(t)=\left[v(t), v_{t}(t)\right] \in C\left([0, T] ; \mathscr{D}\left(\mathscr{A}^{1 / 2}\right) \times \mathscr{D}\left(\mathscr{A}^{1 / 2}\right)\right),
\end{aligned}
$$


where $Z_{\beta}$ is defined in (2.8) and (2.9). The a priori regularity in (3.4)-(3.5) will allow us to justify the computations in the lemmas below. As explained above, in effect, we will work with a set of initial conditions $y_{0, n} \in \mathscr{D}(A)$ given by (3.2c); obtain the desired singular estimate for $\left\|e^{A t} y_{0, n}\right\|$ for this set of initial conditions; and then, at the end, extend the singular estimate to initial conditions of the form $[0,0,0, \mathscr{B} u]$ by letting $z_{n}(0) \rightarrow 0$.

Furthermore, since the proof of Theorem 1.7(II) is more amenable, due to the smoothing effect of the boundary damping on $\Gamma_{0}$ in the wave component, we choose to give first the proof of (II) in Section 3.2. The proof of (I) will require, by contrast, delicate sharp regularity results of the trace $\left.z_{t}\right|_{\Gamma_{0}}$ of a second-order hyperbolic equation, and will be given in Section 3.3.

\subsection{Proof of Theorem $1.7(\mathrm{II})$}

Lemma 3.1. Assume Hypotheses 1.1, 1.2, and 1.3 of (II). Then, the solution of the homogeneous system (3.2) satisfies the following estimate for any $\epsilon>0$, where $\delta_{1}>0$ is the constant in assumption (1.4):

$$
\begin{aligned}
\left\|z_{t}(t)\right\|_{L_{2}(\Omega)}^{2}+ & \left\|A_{N}^{1 / 2} z(t)\right\|_{L_{2}(\Omega)}^{2}+\beta\left\|D_{0}^{1 / 2} N_{0}^{*} A_{N} z(t)\right\|_{L_{2}\left(\Gamma_{0}\right)}^{2} \\
& +\left(2 \delta_{1}-\epsilon\right) \int_{0}^{t}\left\|N_{0}^{*} A_{N} z_{\tau}(\tau)\right\|_{\mathscr{D}\left(\mathscr{A}^{r_{0}}\right)}^{2} d \tau+2 d_{2} \int_{0}^{t}\left\|z_{\tau}(\tau)\right\|_{L_{2}(\Omega)}^{2} d \tau \\
\leq & \frac{1}{\epsilon} \int_{0}^{t}\left\|v_{\tau}(\tau)\right\|_{\left[\mathscr{D}\left(\mathbb{A}^{r_{0}}\right)\right]^{\prime}}^{2} d \tau+\left\|z_{0}\right\|_{Z_{\beta} \cdot}^{2} .
\end{aligned}
$$

(Here and below, we keep $z_{0}$ in the estimates: at the end of this section, we will let a sequence of $z_{0}$ go to zero.)

Proof. With the a priori regularity stated in (3.4), we take the inner product of (3.2a) with $z_{t}$, thus obtaining at each $t$ :

$$
\begin{aligned}
\frac{1}{2} \frac{d}{d t}\{ & \left(z_{t}(t), z_{t}(t)\right)_{L_{2}(\Omega)}+\left(A_{N} z(t), z(t)\right)_{L_{2}(\Omega)} \\
& \left.+\beta\left(D_{0} N_{0}^{*} A_{N} z(t), N_{0}^{*} A_{N} z(t)\right)_{L_{2}\left(\Gamma_{0}\right)}\right\} \\
& +\left(D_{0} N_{0}^{*} A_{N} z_{t}(t), N_{0}^{*} A_{N} z_{t}(t)\right)_{L_{2}\left(\Gamma_{0}\right)}+d_{2}\left(z_{t}(t), z_{t}(t)\right)_{L_{2}(\Omega)} \\
= & \left(v_{t}(t), N_{0}^{*} A_{N} z_{t}(t)\right)_{L_{2}\left(\Gamma_{0}\right)} .
\end{aligned}
$$

Now, integrating (3.7) in time from 0 to $t$ with initial condition $z(0)=z_{0}, z_{t}(0)=$ 0 by (3.2c), and using the left-hand side of estimate (1.4) yields, via Schwarz inequality, 
192 Singular estimates and uniform stability

$$
\begin{aligned}
\left\|z_{t}(t)\right\|_{L_{2}(\Omega)}^{2}+\left\|A_{N}^{1 / 2} z(t)\right\|_{L_{2}(\Omega)}^{2}+\beta\left\|D_{0}^{1 / 2} N_{0}^{*} A_{N} z(t)\right\|^{2} \\
\quad+2 \delta_{1} \int_{0}^{t}\left\|N_{0}^{*} A_{N} z_{\tau}(\tau)\right\|_{\mathscr{D}\left(\mathscr{A}^{r_{0}}\right)}^{2} d \tau+2 d_{2} \int_{0}^{t}\left\|z_{\tau}(\tau)\right\|_{L_{2}(\Omega)}^{2} d \tau \\
\leq 2 \int_{0}^{t}\left|\left(v_{\tau}(\tau), N_{0}^{*} A_{N} z_{\tau}(\tau)\right)_{L_{2}\left(\Gamma_{0}\right)}\right| d \tau \\
\quad+\left\|A_{N}^{1 / 2} z_{0}\right\|_{L_{2}(\Omega)}^{2}+\beta\left\|D_{0}^{1 / 2} N_{0}^{*} A_{N} z_{0}\right\|_{L_{2}\left(\Gamma_{0}\right)}^{2} \\
\leq \epsilon \int_{0}^{t}\left\|N_{0}^{*} A_{N} z_{\tau}(\tau)\right\|_{\mathscr{D}\left(\mathscr{A}^{\left.r_{0}\right)}\right.}^{2} d \tau+\frac{1}{\epsilon} \int_{0}^{t}\left\|v_{\tau}(\tau)\right\|_{\left[\mathscr{D}\left(\mathscr{A}^{r_{0}}\right)\right]^{\prime}}^{2} d \tau+\left\|z_{0}\right\|_{Z_{\beta}}^{2} .
\end{aligned}
$$

In the last step, we have recalled the space $Z_{\beta}$ in (2.8) and (2.9). Then, (3.8) yields (3.6), as desired. Moreover, we have used the duality pairing between $\left[\mathscr{D}\left(\mathscr{A}^{r_{0}}\right)\right]^{\prime}$ and $\mathscr{D}\left(\mathscr{A}^{r_{0}}\right)$ for the last term in (3.7).

As an immediate corollary, we extract the following two key estimates to be invoked in the sequel, by taking now $\epsilon$ sufficiently small in (3.6).

Lemma 3.2. Assume Hypotheses 1.1, 1.2, and 1.3 of Theorem 1.7(II). Then there exists a positive constant $C_{1}$ such that for any $t>0$,

$$
\begin{aligned}
\left\|z_{t}(t)\right\|_{L_{2}(\Omega)}^{2}+\| A_{N}^{1 / 2} z(t) & \left\|_{L_{2}(\Omega)}^{2}+\beta\right\| D_{0}^{1 / 2} N_{0}^{*} A_{N} z(t) \|_{L_{2}\left(\Gamma_{0}\right)}^{2} \\
& \equiv\|z(t)\|_{Z_{\beta}}^{2}+\left\|z_{t}(t)\right\|_{L_{2}(\Omega)}^{2} \\
& \leq C_{1} \int_{0}^{t}\left\|\mathscr{A}^{-r_{0}} v_{\tau}(\tau)\right\|_{L_{2}\left(\Gamma_{0}\right)}^{2} d \tau+\left\|z_{0}\right\|_{Z_{\beta}}^{2} ; \\
\int_{0}^{t}\left\|N_{0}^{*} A_{N} z_{\tau}(\tau)\right\|_{\mathscr{D}\left(\mathscr{A}^{\left.r_{0}\right)}\right.}^{2} d \tau & \leq C_{1} \int_{0}^{t}\left\|\mathscr{A}^{-r_{0}} v_{\tau}(\tau)\right\|_{L_{2}\left(\Gamma_{0}\right)}^{2} d \tau+\left\|z_{0}\right\|_{Z_{\beta}}^{2} .
\end{aligned}
$$

At this point we need to estimate the integral on the right-hand side of (3.9) and (3.10) in terms of $\mathscr{A}^{-r} v_{2} \in L_{2}\left(\Gamma_{0}\right)$.

Lemma 3.3. Assume, in addition to Hypotheses 1.1, 1.2, and 1.3, also condition (1.9): $\alpha-2 r \geq-2 r_{0}$ for the constants $r, r_{0}$, and $\alpha$ as in Theorem 1.7(II). Then, the following estimate holds true for the component $v_{t}$ of system (3.2), where $C_{2}$ and $C_{3}$ are suitable (generic) positive constants:

(i)

$$
\int_{0}^{t}\left\|\mathscr{A}^{-r_{0}} v_{\tau}(\tau)\right\|_{L_{2}\left(\Gamma_{0}\right)}^{2} d \tau \leq C_{3} e^{C_{2} t}\left[\left\|\mathscr{A}^{-r} v_{2}\right\|_{L_{2}\left(\Gamma_{0}\right)}^{2}+\left\|z_{0}\right\|_{Z_{\beta}}^{2}\right] .
$$

(ii) Consequently, by (3.10) and (3.11),

$$
\int_{0}^{t}\left\|N_{0}^{*} A_{N} z_{\tau}(\tau)\right\|_{\mathscr{D}\left(\mathscr{A}^{\left.r_{0}\right)}\right.}^{2} d \tau \leq C e^{C_{2} t}\left[\left\|\mathscr{A}^{-r} v_{2}\right\|_{L_{2}\left(\Gamma_{0}\right)}^{2}+\left\|z_{0}\right\|_{Z_{\beta}}^{2}\right] .
$$


Remark 3.4. If we had $\alpha=1$ in the plate model (1.2d) (Kelvin-Voight damping), then by (1.9), we could then take $r_{0}=0$ in assumption (1.4), and so we could as well deal with a bounded damping operator $D_{0}=d_{0} I, d_{0} \geq 0$. In the present Part (II) of boundary damping on $\Gamma_{0}$, we would then have $d_{0}>0$. Then, the proof of Lemma 3.3, (3.11), in the case $r_{0}=0$ would simplify, as it would only require to have an estimate of $v_{t} \in L_{2}\left(0, t ; L_{2}\left(\Gamma_{0}\right)\right)$ in terms of $\mathscr{A}^{-r} v_{2} \in L_{2}\left(\Gamma_{0}\right)$. This is an easier task over the one of establishing the full statement of (3.11) when $r_{0}>0$. In short, the weakening of the damping term $\mathscr{A}^{\alpha} v_{t}$ in the plate equation (1.2d), as $\alpha$ decreases from $\alpha=1\left(r_{0}=0\right)$ to $\alpha=1 / 2\left(r_{0}>0\right.$ as in (1.4) and (1.5)) forces the necessity of increasing the strength of the damping operator $D_{0}\left(r_{0}\right)$ on the wave equation in (1.2c). This is then responsible for requiring the full strength of the statement of Lemma 3.3, which then reflects the additional difficulty caused by the parameter $\alpha$, as it ranges from $\alpha=1$ (easier case) to $\alpha=1 / 2$ (more challenging case).

Proof of Lemma 3.3. We will provide two different proofs of this critical new ingredient over the case $\alpha=1$ : one here below, and one in Appendix B.

First proof. (i) With the a priori regularity stated in (3.3), (3.4), and (3.5), we take the inner product of (3.2b) with $\mathscr{A}^{-2 r} v_{t}$, where $r$ is the constant of assumption (1.3). We then obtain at each $t$ the following equality:

$$
\begin{aligned}
\frac{1}{2} \frac{d}{d t}\{ & \left.\left\|\mathscr{A}^{-r} v_{t}(t)\right\|^{2}+\left\|\mathscr{A}^{1 / 2-r} v(t)\right\|^{2}\right\} \\
& +\rho\left\|\mathscr{A}^{\alpha / 2-r} v_{t}(t)\right\|^{2}+\left(N_{0}^{*} A_{N} z_{t}(t), \mathscr{A}^{-2 r} v_{t}(t)\right)=0 .
\end{aligned}
$$

Now, we integrate (3.13) in time from 0 to $t$ with initial condition $v(0)=0$, $v_{t}(0)=v_{2}$ by $(3.2 \mathrm{c})$ - using Schwarz inequality. We obtain

$$
\begin{aligned}
&\left\|\mathscr{A}^{-r} v_{t}(t)\right\|_{L_{2}\left(\Gamma_{0}\right)}^{2}+\left\|\mathscr{A}^{1 / 2-r} v(t)\right\|_{L_{2}\left(\Gamma_{0}\right)}^{2}+2 \rho \int_{0}^{t}\left\|\mathscr{A}^{\alpha / 2-r} v_{\tau}(\tau)\right\|_{L_{2}\left(\Gamma_{0}\right)}^{2} d \tau \\
& \leq 2 \int_{0}^{t}\left|\left(\mathscr{A}^{r_{0}} N_{0}^{*} A_{N} z_{\tau}(\tau), \mathscr{A}^{-2 r-r_{0}} v_{\tau}(\tau)\right)_{L_{2}\left(\Gamma_{0}\right)}\right| d \tau+\left\|\mathscr{A}^{-r} v_{2}\right\|_{L_{2}\left(\Gamma_{0}\right)}^{2} \\
& \leq \epsilon \int_{0}^{t}\left\|N_{0}^{*} A_{N} z_{\tau}(\tau)\right\|_{\mathscr{D}\left(\mathscr{A}^{r}\right)}^{2} d \tau \\
&+\frac{1}{\epsilon} \int_{0}^{t}\left\|\mathscr{A}^{-2 r-r_{0}} v_{\tau}(\tau)\right\|_{L_{2}\left(\Gamma_{0}\right)}^{2} d \tau+\left\|\mathscr{A}^{-r} v_{2}\right\|_{L_{2}\left(\Gamma_{0}\right)}^{2}
\end{aligned}
$$

$$
\begin{aligned}
\text { (by }(3.10)) \leq & C_{1} \epsilon \int_{0}^{t}\left\|\mathscr{A}^{-r_{0}} v_{\tau}(\tau)\right\|_{L_{2}\left(\Gamma_{0}\right)}^{2} d \tau \\
& +\frac{1}{\epsilon} \int_{0}^{t}\left\|\mathscr{A}^{-2 r-r_{0}} v_{\tau}(\tau)\right\|_{L_{2}\left(\Gamma_{0}\right)}^{2} d \tau \\
& +\left\|\mathscr{A}^{-r} v_{2}\right\|_{L_{2}\left(\Gamma_{0}\right)}^{2}+\epsilon\left\|z_{0}\right\|_{Z_{\beta}}^{2},
\end{aligned}
$$

where to go from (3.14) to (3.15) we have invoked estimate (3.10) for $N_{0}^{*} A_{N} z_{t}$. 
Next, we recall assumption (1.9) of the present part (II) that

$$
\frac{\alpha}{2}-r \geq-r_{0}, \quad \text { so that } \quad\left\|\mathscr{A}^{\alpha / 2-r} v_{\tau}(\tau)\right\|^{2} \geq c_{1}\left\|\mathscr{A}^{-r_{0}} v_{\tau}(\tau)\right\|^{2},
$$

where here and below, in the present proof, $\|\quad\|$ refers to the $L_{2}\left(\Gamma_{0}\right)$-norm. As a consequence, using this bound on the third term on the left-hand side of (3.14), we see that (3.15) implies

$$
\begin{aligned}
& \left\|\mathscr{A}^{-r} v_{t}(t)\right\|^{2}+\left\|\mathscr{A}^{1 / 2-r} v(t)\right\|^{2}+2 \rho c_{1} \int_{0}^{t}\left\|\mathscr{A}^{-r_{0}} v_{\tau}(\tau)\right\|^{2} d \tau \\
& \quad \leq C_{1} \epsilon \int_{0}^{t}\left\|\mathscr{A}^{-r_{0}} v_{\tau}(\tau)\right\|^{2} d \tau+\frac{1}{\epsilon} \int_{0}^{t}\left\|\mathscr{A}^{-2 r-r_{0}} v_{\tau}(\tau)\right\|^{2} d \tau+\left\|\mathscr{A}^{-r} v_{2}\right\|^{2}+\epsilon\left\|z_{0}\right\|_{Z_{\beta}}^{2} .
\end{aligned}
$$

Taking now $\epsilon$ sufficiently small, and since $-r>-2 r-r_{0}$, we obtain, with positive constants $C_{2}$ and $c_{2}=2 \rho c_{1}-C_{1} \epsilon>0$,

$$
\begin{array}{r}
\left\|\mathscr{A}^{-r} v_{t}(t)\right\|^{2}+\left\|\mathscr{A}^{1 / 2-r} v(t)\right\|^{2}+c_{2} \int_{0}^{t}\left\|\mathscr{A}^{-r_{0}} v_{\tau}(\tau)\right\|^{2} d \tau \\
\leq C_{2} \int_{0}^{t}\left\|\mathscr{A}^{-r} v_{\tau}(\tau)\right\|^{2} d \tau+\left\|\mathscr{A}^{-r} v_{2}\right\|^{2}+\epsilon\left\|z_{0}\right\|_{Z_{\beta}}^{2},
\end{array}
$$

which finally yields

$$
\left\|\mathscr{A}^{-r} v_{t}(t)\right\|^{2} \leq\left\|\mathscr{A}^{-r} v_{2}\right\|^{2}+\epsilon\left\|z_{0}\right\|_{Z_{\beta}}^{2}+C_{2} \int_{0}^{t}\left\|\mathscr{A}^{-r} v_{\tau}(\tau)\right\|^{2} d \tau .
$$

In (3.19), we invoke the classical Gronwall inequality (see [37, page 92], [29, page 205]), thus obtaining

$$
\left\|\mathscr{A}^{-r} v_{t}(t)\right\|^{2} \leq\left[\left\|\mathscr{A}^{-r} v_{2}\right\|^{2}+\epsilon\left\|z_{0}\right\|_{Z_{\beta}}^{2}\right] e^{C_{2} t}
$$

which in turn implies after integration

$$
\int_{0}^{t}\left\|\mathscr{A}^{-r} v_{\tau}(\tau)\right\|^{2} d \tau \leq \frac{1}{C_{2}}\left(e^{C_{2} t}-1\right)\left[\left\|\mathscr{A}^{-r} v_{2}\right\|^{2}+\epsilon\left\|z_{0}\right\|_{Z_{\beta}}^{2}\right] .
$$

We return to (3.18), apply (3.21) on its right-hand side and finally get

$$
\begin{aligned}
\int_{0}^{t}\left\|\mathscr{A}^{-r_{0}} v_{\tau}(\tau)\right\|^{2} d \tau & \leq \frac{1}{c_{2}}\left[\left(e^{C_{2} t}-1\right)+1\right]\left\{\left\|\mathscr{A}^{-r} v_{2}\right\|^{2}+c\left\|z_{0}\right\|_{Z_{\beta}}^{2}\right\} \\
& =\frac{1}{c_{2}} e^{C_{2} t}\left[\left\|\mathscr{A}^{-r} v_{2}\right\|^{2}+c\left\|z_{0}\right\|_{Z_{\beta}}^{2}\right],
\end{aligned}
$$

which is nothing but (3.11), as desired, after setting $C_{3}=1 / c_{2}$.

(ii) Estimate (3.12) follows immediately from (3.10) by using (3.11).

A second proof of Lemma 3.3 is given in Appendix B. 
Proposition 3.5. Assume Hypotheses 1.1, 1.2, and 1.3, and, in addition, condition (1.9): $\alpha-2 r \geq-2 r_{0}$ of Theorem 1.7(II). Then, the solution $y(t)=\left[y_{1}(t), y_{2}(t)\right]$ of system (3.1) or (3.2), with $y_{1}(t)=\left[z(t), z_{t}(t)\right], y_{2}(t)=\left[v(t), v_{t}(t)\right]$, satisfies the following estimates on $0 \leq t \leq T$, where the constant $\gamma$ is given in (1.10) and $C_{i}$ are suitable positive constants:

$$
\begin{aligned}
& \left\|y_{1}(t)\right\|_{Y_{1}}^{2}=\left\|\left[z(t), z_{t}(t)\right]\right\|_{Y_{1}}^{2} \leq C_{1} e^{C_{2} t}\left[\left\|\mathscr{A}^{-r} v_{2}\right\|_{L_{2}\left(\Gamma_{0}\right)}^{2}+\left\|z_{0}\right\|_{Z_{\beta}}^{2}\right] \\
& \left\|y_{2}(t)\right\|_{Y_{2}}=\left\|\left[v(t), v_{t}(t)\right]\right\|_{Y_{2}} \leq C_{T} e^{C_{2} t}\left(\frac{1}{t^{\gamma}}+1\right)\left[\left\|\mathscr{A}^{-r} v_{2}\right\|_{L_{2}\left(\Gamma_{0}\right)}+\left\|z_{0}\right\|_{Z_{\beta}}\right] .
\end{aligned}
$$

In summary, the following is true (see (3.2c)):

$$
\begin{aligned}
\left\|e^{A t} y(0)\right\|_{Y} & =\left\|e^{A t}\left[\begin{array}{c}
z_{0} \\
0 \\
0 \\
v_{2}
\end{array}\right]\right\|_{Y}=\left\|\left[\begin{array}{l}
y_{1}(t) \\
y_{2}(t)
\end{array}\right]\right\|_{Y} \\
& \leq \frac{C_{T}}{t^{\gamma}}\left\{\left\|z_{0}\right\|_{Z_{\beta}}+\left\|\mathscr{A}^{-r} v_{2}\right\|_{L_{2}\left(\Gamma_{0}\right)}\right\}, \quad 0<t \leq T,
\end{aligned}
$$

for all $z_{0} \in Z_{\beta}$, and all $v_{2} \in\left[\mathscr{D}\left(\mathscr{A}^{r}\right)\right]^{\prime}$. (The factor $e^{C_{2} t}$ in (3.23), (3.24) is not important.)

Proof. The first estimate (3.23) is easily deduced from (3.9) by using (3.11) and recalling (2.10) for $Y_{1}$ and (2.8) and (2.9) for $Z_{\beta}$. In order to obtain the second estimate (3.24), we return to the initial value problem (3.2), with focus on the $v$-equation (3.2b). By the structure of the operator $A$ in (2.37) and (2.38), it follows that this equation, $(3.2 \mathrm{~b})$, reduces to the first-order system

$$
\frac{d}{d t}\left[\begin{array}{c}
v \\
v_{t}
\end{array}\right]=A_{2}\left[\begin{array}{c}
v \\
v_{t}
\end{array}\right]+\left[\begin{array}{c}
0 \\
-N^{*} A_{N} z_{t}
\end{array}\right], \quad\left[\begin{array}{c}
v(0) \\
v_{t}(0)
\end{array}\right]=\left[\begin{array}{c}
0 \\
v_{2}
\end{array}\right],
$$

whose mild solution is given by

$$
\left[\begin{array}{c}
v(t) \\
v_{t}(t)
\end{array}\right]=e^{A_{2} t}\left[\begin{array}{c}
0 \\
v_{2}
\end{array}\right]-\int_{0}^{t} e^{A_{2}(t-\tau)}\left[\begin{array}{c}
0 \\
N^{*} A_{N} z_{\tau}(\tau)
\end{array}\right] d \tau
$$

We recall that, by Lemma 2.7, the operator $A_{2}$ in (3.26) and (3.27) is the generator of an s.c. semigroup of contractions $e^{A_{2} t}$ on the space $Y_{2}$, which, moreover, is analytic on $Y_{2}$. We split the first term on the right-hand side of (3.27) as

$$
\left\|e^{A_{2} t}\left[\begin{array}{c}
0 \\
v_{2}
\end{array}\right]\right\|=\left\|\left(-A_{2}\right)^{s} e^{A_{2} t}\left(-A_{2}\right)^{-s}\left[\begin{array}{c}
0 \\
v_{2}
\end{array}\right]\right\|,
$$


with $s$ to be chosen appropriately below: this way we can exploit both analyticity of $e^{A_{2} t}$ and the estimates preliminarily established in Lemma 2.8 for the fractional powers $A_{2}^{-s}$. To proceed, we need to distinguish two cases.

Case 1. Let $r / \alpha \leq 1 / 2$. Then we take $s=r / \alpha$ in (3.28), thus apply formula (2.26) and immediately get

$$
\begin{aligned}
\left\|e^{A_{2} t}\left[\begin{array}{c}
0 \\
v_{2}
\end{array}\right]\right\|_{Y_{2}} & =\left\|\left(-A_{2}\right)^{r / \alpha} e^{A_{2} t}\left(-A_{2}\right)^{-r / \alpha}\left[\begin{array}{c}
0 \\
v_{2}
\end{array}\right]\right\|_{Y_{2}} \\
& \leq\left\|\left(-A_{2}\right)^{r / \alpha} e^{A_{2} t}\right\|\left\|\mathscr{A}^{-r} v_{2}\right\|_{L_{2}\left(\Gamma_{0}\right)} \leq \frac{c_{3}}{t^{r / \alpha}}\left\|\mathscr{A}^{-r} v_{2}\right\|_{L_{2}\left(\Gamma_{0}\right)},
\end{aligned}
$$

where $c_{3}$ is a positive constant.

Case 2. Let instead $r / \alpha>1 / 2$, we then seek to find an exponent $s$, which will turn out to be $1 / 2<s<1$, such that, by (2.28), the following estimates hold true:

$$
\begin{aligned}
\left\|A_{2}^{-s}\left[\begin{array}{c}
0 \\
v_{2}
\end{array}\right]\right\|_{Y_{2}}^{2} & \leq k\left\|\mathscr{A}^{1 / 2-\alpha+s(\alpha-1)} v_{2}\right\|_{L_{2}\left(\Gamma_{0}\right)}^{2}=k\left\|\mathscr{A}^{1 / 2-\alpha+r+s(\alpha-1)} \mathscr{A}^{-r} v_{2}\right\|_{L_{2}\left(\Gamma_{0}\right)}^{2} \\
& \leq c^{2}\left\|\mathscr{A}^{-r} v_{2}\right\|_{L_{2}\left(\Gamma_{0}\right)}^{2} .
\end{aligned}
$$

This is possible, provided that $1 / 2-\alpha+r+s(\alpha-1)=0$ (or $\leq 0)$, that is, provided that we choose

$$
s=\gamma \equiv \frac{1 / 2-\alpha+r}{1-\alpha}, \quad \text { so that } \quad \frac{1}{2}=\frac{1 / 2-\alpha+\alpha / 2}{1-\alpha}<s=1+\frac{r-1 / 2}{1-\alpha}<1,
$$

as anticipated, in which case estimate (3.31) holds true. Therefore, with $s=\gamma$ given by (3.32), that is, with $\gamma$ given by (1.10), returning to (3.28) and using (3.31), we obtain

$$
\begin{aligned}
\left\|e^{A_{2} t}\left[\begin{array}{c}
0 \\
v_{2}
\end{array}\right]\right\|_{Y_{2}} & =\left\|\left(-A_{2}\right)^{s} e^{A_{2} t}\left(-A_{2}\right)^{-s}\left[\begin{array}{c}
0 \\
v_{2}
\end{array}\right]\right\|_{Y_{2}} \\
& \leq\left\|\left(-A_{2}\right)^{s} e^{A_{2} t}\right\|\left\|\left(-A_{2}\right)^{-s}\left[\begin{array}{c}
0 \\
v_{2}
\end{array}\right]\right\|_{Y_{2}} \\
(\operatorname{by}(3.31)) & \leq \frac{k c_{3}}{t^{\gamma}}\left\|\mathscr{A}^{-r} v_{2}\right\|_{L_{2}\left(\Gamma_{0}\right)}, \quad 0<t,
\end{aligned}
$$

as desired.

We notice that for $r / \alpha=1 / 2$ both cases yield the same estimate $\gamma=1 / 2$. On the other hand, in both cases $r / \alpha \leq 1 / 2$ and $r / \alpha>1 / 2$, we obtain by use of Hölder's 
inequality, estimate (3.12), and (2.10) on $Y_{2}$ :

$$
\begin{aligned}
\left\|\int_{0}^{t} e^{A_{2}(t-\tau)}\left[\begin{array}{c}
0 \\
N^{*} A_{N} z_{\tau}(\tau)
\end{array}\right] d \tau\right\|_{Y_{2}} & \leq \int_{0}^{t}\left\|N^{*} A_{N} z_{\tau}(\tau)\right\|_{L_{2}\left(\Gamma_{0}\right)} d \tau \\
& \leq \sqrt{t}\left(\int_{0}^{t}\left\|N^{*} A_{N} z_{\tau}(\tau)\right\|_{L_{2}\left(\Gamma_{0}\right)}^{2} d \tau\right)^{1 / 2} \\
(\operatorname{by}(3.12)) & \leq\left[\left\|\mathscr{A}^{-r} v_{2}\right\|_{L_{2}\left(\Gamma_{0}\right)}+\left\|z_{0}\right\|_{Z_{\beta}}\right] c_{5} \sqrt{t} e^{\left(C_{2} / 2\right) t} \\
& \leq\left[\left\|\mathscr{A}^{-r} v_{2}\right\|_{L_{2}\left(\Gamma_{0}\right)}+\left\|z_{0}\right\|_{Z_{\beta}}\right] c_{T} e^{C_{2} t},
\end{aligned}
$$

$0<t \leq T$, for a suitable constant $c_{T}$.

Finally, in order to estimate the solution (3.27), we combine (3.29), (3.33), and (3.34) and obtain (3.24), as desired. Finally, estimate (3.25) is obtained by continuous extension, from $y(0) \in \mathscr{D}(A)$ as originally taken in $(3.2 \mathrm{c})$ to $y(0) \in$ $Y$.

We finally complete the proof of Theorem 1.7 (II) by the anticipated limit process described in Section 3.1. (That is why we kept $z_{0}$ in the estimates throughout.)

Completion of the proof of Theorem 1.7(II). We want to show that, under Assumptions 1.1, 1.2, 1.3, and, in addition, condition (1.9): $\alpha-2 r \geq-2 r_{0}$ of part (II), then the singular estimate (1.8) holds true, where the exponent $\gamma$ is defined by $(1.10)$.

To establish this, we consider a sequence of problems (3.2) with initial conditions $y_{n}(0)=\left\{z_{n, 0}, 0,0, v_{2}\right\} \in \mathscr{D}(A)$, and $z_{n, 0} \rightarrow 0$ in $Z_{\beta}$. For each such problem, Proposition 3.5 holds true, and (3.25) yields, for each $t>0$,

$$
\begin{aligned}
\left\|e^{A t} y_{n}(0)\right\|_{Y} & =\left\|e^{A t}\left[\begin{array}{c}
z_{n, 0} \\
0 \\
0 \\
v_{2}
\end{array}\right]\right\|_{Y} \\
& \leq \frac{C_{T}}{t^{\gamma}}\left\{\left\|z_{n, 0}\right\|_{Z_{\beta}}+\left\|\mathscr{A}^{-r} v_{2}\right\|_{L_{2}\left(\Gamma_{0}\right)}\right\}, \quad 0<t \leq T,
\end{aligned}
$$

for all $z_{n, 0} \in Z_{\beta}$ and all $v_{2} \in\left[\mathscr{D}\left(\mathscr{A}^{r}\right)\right]^{\prime}$. Letting $n \nearrow \infty$ on both sides of (3.35), we obtain, with $y(0)=\left[0,0,0, v_{2}\right]$,

$$
\left\|e^{A t} y(0)\right\|_{Y}=\left\|e^{A t}\left[\begin{array}{c}
0 \\
0 \\
0 \\
v_{2}
\end{array}\right]\right\|_{Y} \leq \frac{C_{T}}{t^{\gamma}}\left\|\mathscr{A}^{-r} v_{2}\right\|_{L_{2}\left(\Gamma_{0}\right)}, \quad 0<t \leq T,
$$


for all $v_{2} \in\left[\mathscr{D}\left(\mathscr{A}^{r}\right)\right]^{\prime}$. Setting, in particular, $v_{2}=\mathscr{B} u$ in (3.36), that is, taking $y(0)=B \tilde{u}=[0,0,0, \mathscr{B} u]$, we obtain by recalling (1.3),

$$
\left\|e^{A t} B \tilde{u}\right\|_{Y} \leq \frac{C_{T}}{t^{\gamma}}\left\|\mathscr{A}^{-r} \mathscr{B} u\right\|_{L_{2}\left(\Gamma_{0}\right)}=\frac{C_{T}}{t^{\gamma}}\|u\|_{u}=\frac{C_{T}}{t^{\gamma}}\|\tilde{u}\|_{U}, \quad 0<t \leq T,
$$

and Theorem 1.7(II) is proved.

\subsection{Proof of Theorem 1.7(I): case $D_{0}=0$}

Orientation. Theorem 1.7 says that under the assumptions of part (I), we can take the operator $D_{0}$ in $(1.2 \mathrm{c})$ to be null: $D_{0} \equiv 0$. This means that the boundary condition on $\Gamma_{0}$ does not provide in this case any smoothing effect for the velocity trace $\left.z_{t}\right|_{\Gamma_{0}}=N_{0}^{*} A_{N} z_{t}$ of the wave component on the flat, flexible wall $\Gamma_{0}$. Thus, the present case $D_{0}=0$, where no regularizing damping occurs on the interface wall $\Gamma_{0}$ for the $z$-problem, is much more challenging than the one of Section 3.2, where-by contrast-a natural regularity of the trace $\left.z_{t}\right|_{\Gamma_{0}}$ in (1.2c) was built-in with $D_{0}$ coercive and satisfying assumption (1.4). In the present case, in fact, the a priori regularity of the velocity component $z_{t} \in L_{2}(\Omega)$ (due to Theorem 1.6) does not even allow a priori to define the trace $\left.z_{t}\right|_{\Gamma_{0}}$ on the boundary $\Gamma_{0}$. On the other hand, this trace appears in (1.2c) of the model, as a coupling term between the abstract plate and the wave. This coupling term is now the main technical difficulty of the problem under study in the present case $D_{0}=0$. In order to cope with this issue, we will have to resort to "sharp" trace regularity theory for solutions to hyperbolic problems [19, 21, 22, 34] (see also [2] for a parallelopiped). It is known, by now, that hyperbolicity in the dynamics induces a certain additional regularity to traces of solutions on the boundary, which is not obtainable from trace theory and sharp interior regularity. The amount of additional regularity gained on the boundary depends on the boundary conditions imposed on the wave. More precisely,

(a) if the boundary conditions of the hyperbolic problem are of Dirichlet type (i.e., the so-called Lopatinski condition is satisfied), then traces of its solutions on the boundary display an additional $1 / 2$ derivative with respect to standard interior results $[17,19]$. In particular, finite energy solutions $z \in H^{1}(Q)$ will have traces $\left.z\right|_{\Gamma} \in H^{1}(\Sigma)$. This means that we obtain a gain of $1 / 2$ derivative with respect to classical trace theory applied to $H^{1}(\Omega)$-functions;

(b) if, instead, the boundary conditions do not satisfy the Lopatinski condition (as in the present case of Neumann BC), then there is still a gain of boundary (trace) regularity of hyperbolic solutions, but always strictly less than $1 / 2$, unless the wave equation is one dimensional. The amount of the "additional" regularity gained on the boundary depends on the geometry of the domain. 
For instance, to illustrate, if $\Omega$ is an $n$-dimensional parallelopiped (resp., an $n$-sphere), $n \geq 2$, then a finite energy wave-equation solution $z \in H^{1}(Q)$ has a trace $\left.z\right|_{\Sigma} \in H^{3 / 4}(\Sigma)$ (resp., $\left.z\right|_{\Sigma} \in H^{2 / 3}(\Sigma)$ ) (see [23, Theorems B and C] and [23, Theorem 3.3a]), thus with a gain of $1 / 4$ (resp., of 1/6) with respect to standard trace theory applied to interior regularity. The case of a general domain $\Omega, \operatorname{dim}$ $\Omega \geq 2$, is like that of the sphere [21, Theorems 1.2b, 1.3b] and [34].

Remark 3.6. It appears that in our present context, where the trace of the wave solution $z$ is needed globally on all of the flat boundary $\Gamma_{0}$, we cannot appeal to the local trace regularity on flat surfaces as in [34], which is like the global regularity of the parallelopiped, as we approach the junction between $\Gamma_{0}$ and $\Gamma_{1}$ (which is not flat). This accounts for the introduction of the parameter " $a$ " in (1.7b), which distinguishes between a parallelopiped $\Omega$ (where the trace results are better) and a general domain $\Omega$.

Remark 3.7. (Critical importance to have a regularity theory: (3.45), (3.46), (3.47), (3.48), (3.49), (3.50), and (3.52) below for the Neumann mixed problems (3.44) with the parameter $a$ strictly greater than $1 / 2: a>1 / 2$.) The goal of this remark is to show that:

(i) if the regularity theory (3.45), (3.46), (3.47), (3.48), (3.49), (3.50), and (3.52) of the Neumann mixed problems (3.44) were available only in the case $a=1 / 2$, then (our proof of Theorem 1.7(I) would fail and) we could not assert the validity of Theorem 1.7 in the canonical case where $\mathscr{A}$ and $\mathscr{B}$ are given by (1.11) and (1.12);

(ii) on the other hand, if the regularity theory (3.45), (3.46), (3.47), (3.48), (3.49), (3.50), and (3.52) of the Neumann mixed problem (3.44) were available only in the case $a=1 / 2+\epsilon$, for all $\epsilon>0$, then we could assert the validity of Theorem 1.7(I) only in the case $\alpha=1$ for $\mathscr{A}$ and $\mathscr{B}$ given by (1.11), (1.12). Naturally, from (1.7a), the higher the parameter $a$, the lower the parameter $\theta_{0}$, the larger the range of $\alpha \leq 1$ for the validity of Theorem 1.7(I).

Justification of (i). Let $a=1 / 2$, so that $H^{1-a}\left(\Gamma_{0}\right) \equiv H^{1 / 2}\left(\Gamma_{0}\right)$. Let $\mathscr{A}$ and $\mathscr{B}$ be given by (1.11) and (1.12), so that $r=3 / 8+\epsilon$, by (1.13).

(a) Assume that $\mathscr{A}$ includes hinged or clamped BC in this case, there is no $\theta_{0}$ such that $H^{1-a}\left(\Gamma_{0}\right) \equiv H^{1 / 2}\left(\Gamma_{0}\right)=\mathscr{D}\left(\mathscr{A}^{\theta_{0}}\right)$. Indeed, for the candidate value $\theta_{0}=1 / 8$ we have [10], [24, page 284],

$$
\mathscr{D}\left(\mathscr{A}^{1 / 8}\right)=H_{00}^{1 / 2}\left(\Gamma_{0}\right) \varsubsetneqq H^{1 / 2}\left(\Gamma_{0}\right) .
$$

Thus, in this case, the first part of assumption (1.7a) is violated.

(b) Assume now that $\mathscr{A}$ includes the free BC Then, the first part of assumption (1.7a) is satisfied with $\theta_{0}=1 / 8$ [10], [24, page 284]:

$$
H^{1-a}\left(\Gamma_{0}\right) \equiv H^{1 / 2}\left(\Gamma_{0}\right) \equiv \mathscr{D}\left(\mathscr{A}^{\theta_{0}}\right)=\mathscr{D}\left(\mathscr{A}^{1 / 2}\right) \text {. }
$$


However, in this case where $r=3 / 8+\epsilon$ by (1.13), we have that the second part of assumption (1.7a) imposes the condition $\alpha \geq 2 r+\theta_{0}=2(3 / 8+\epsilon)+1 / 8=1+2 \epsilon$, $\epsilon>0$. Thus, such condition is not satisfied in the range $1 / 2 \leq \alpha \leq 1$.

Justification of (ii). Let now $a=1 / 2+\epsilon$, for all $\epsilon>0$. Then, in this case, the first part of assumption (1.7a) is satisfied with $\theta_{0}=1 / 8-\epsilon / 4$ [10], [24, page 284],

$$
H^{1-a}\left(\Gamma_{0}\right)=H^{1 / 2-\epsilon}\left(\Gamma_{0}\right)=\mathscr{D}\left(\mathscr{A}^{1 / 8-\epsilon / 4}\right),
$$

for all BC (hinged, clamped, free, etc.). Then, with $\theta_{0}=1 / 8-\epsilon / 4$, for all $\epsilon>0$ and $r=3 / 8+\epsilon^{\prime}$, for all $\epsilon^{\prime}>0$, we see that we can satisfy the second part of assumption (1.7a) only for the case $\alpha=1$.

We finally note that the "classical" regularity theory $[27,28,30]$ for the Neumann mixed problems (3.44) does not even guarantee the validity of (3.45), (3.46), (3.47), (3.48), (3.49), (3.50), and (3.52) for $a=1 / 2$. Thus, the availability of the sharp/optimal theory for problems (3.44) given in [21, 22, 23, 34] (see also [1] for a parallelopiped) is critical to obtain Theorem 1.7(I).

Proof of Theorem 1.7(I)

Step 1. As mentioned in the above Orientation, the proof of Theorem 1.7(I) relies on "sharp" regularity results obtained for traces of wave equations subject to Neumann's data. By exploiting this particular regularity (which does not follow from trace theory), we are able, in the range $2 \theta_{0}+2 r \leq \alpha \leq 1$, to dispense altogether with the necessity of additional damping on the wall $\Gamma_{0}$ and choose $D_{0}=0$. Accordingly, we consider the following wave equation:

$$
\begin{array}{ll}
z_{t t}=\Delta z-d_{2} z_{t} & \text { in }(0, T] \times \Omega=Q ; \\
z(0, \cdot)=z_{0}, \quad z_{t}(0, \cdot)=z_{1} & \text { in } \Omega ; \\
\frac{\partial z}{\partial v}+d_{1} z=0 & \text { in }(0, T] \times \Gamma_{1}=\Sigma_{1} ; \\
\frac{\partial z}{\partial v}=g & \text { on }(0, T] \times \Gamma_{0}=\Sigma_{0} .
\end{array}
$$

The required sharp trace regularity result is given next.

Proposition 3.8. Let $z$ be a finite energy solution of problem (3.41), where $d_{2} \in \mathbb{R}$, $d_{1} \geq 0$. Recall the parameter a given in (1.7b): $a=3 / 4$ for $\Omega$ being a parallelopiped; and $a=2 / 3$ for a general domain $\Omega$ (which, moreover, is smooth or convex, as noted in the paragraph above problem (1.2)). Then there exists a constant $C_{T}>0$ such that

$$
\int_{0}^{T}\left\|z_{t}\right\|_{H^{a-1}\left(\Gamma_{0}\right)}^{2} d t \leq C_{T}\left[\left\|z_{0}\right\|_{H^{1}(\Omega)}^{2}+\left\|z_{1}\right\|_{L_{2}(\Omega)}^{2}+\int_{0}^{T}\|g\|_{H^{1-a}\left(\Gamma_{0}\right)}^{2} d t\right] .
$$

Proof. The trace regularity result of Proposition 3.8 is essentially given in the above references $[2,20,21,22,23,34]$. Given the critical role of this result in 
the present proof of Theorem 1.7(I), we will provide some details based on general arguments. In [2], the result for a parallelopiped by a directed computation based on eigenfunction expansion is given. By the superposition principle, we write

$$
\begin{array}{lll} 
& z=\zeta+w ; & \\
\zeta_{t t}=\Delta \zeta-d_{2} \zeta_{t} ; & w_{t t}=\Delta w-d_{2} w_{t} & \text { in } Q ; \\
\zeta(0, \cdot)=z_{0}, \quad \zeta_{t}(0, \cdot)=z_{1} ; & w(0, \cdot)=0, \quad w_{t}(0, \cdot)=0 & \text { in } \Omega ; \\
\frac{\partial \zeta}{\partial v}+d_{1} \zeta=0 ; & \frac{\partial w}{\partial v}+d_{1} w=0 & \text { in } \Sigma_{1} ; \\
\frac{\partial \zeta}{\partial v}=0 ; & \frac{\partial w}{\partial v}=g & \text { in } \Sigma_{0} .
\end{array}
$$

Analysis of $\zeta$-problem (3.44). According to [22, Theorems B and C], [23, Theorem 3.3], and [34] we have that, the map

$$
\begin{aligned}
\left\{z_{0}, z_{1}\right\} & \in H^{1}(\Omega) \times\left. L_{2}(\Omega) \longrightarrow \zeta\right|_{\Sigma_{0}} \in H^{a}\left(\Sigma_{0}\right) \\
& \equiv H^{a}\left(0, T ; L_{2}\left(\Gamma_{0}\right)\right) \cap L_{2}\left(0, T ; H^{a}\left(\Gamma_{0}\right)\right)
\end{aligned}
$$

is continuous, with $a=3 / 4$ when $\Omega$ is a parallelopiped and $a=2 / 3$ for a general $\Omega$, as assumed. It then follows a fortiori from (3.45) that

$$
\left.\zeta_{t}\right|_{\Sigma_{0}} \in H^{a-1}\left(0, T ; L_{2}\left(\Gamma_{0}\right)\right) \text { continuously in }\left\{z_{0}, z_{1}\right\} \in H^{1}(\Omega) \times L_{2}(\Omega) \text {. }
$$

On the other hand, we know from $[20,21,22,23,34]$ that for the Neumann mixed hyperbolic problem (3.41), the loss of differentiability occurs only in the characteristic microlocal sector. We denote by $H_{\mathrm{cs}}^{\ell}$ and $H_{\mathrm{ncs}}^{\ell}$ the microlocal regularity in the characteristic sector, and in the non-characteristic sector, respectively. With reference to the $\zeta$-problem in (3.44), we have

$$
\zeta \in H_{\mathrm{ncs}}^{1}\left(\Sigma_{0}\right) \text {, hence }\left.\zeta_{t}\right|_{\Sigma_{0}} \in H_{\mathrm{ncs}}^{0}\left(\Sigma_{0}\right) \text {. }
$$

Combining (3.46) and (3.47), we can restate the regularity of $\left.\zeta_{t}\right|_{\Sigma_{0}}$ more precisely as

$$
\left.\zeta_{t}\right|_{\Sigma_{0}} \in H_{\mathrm{ncs}}^{0}\left(\Sigma_{0}\right) \cap H^{a-1}\left(0, T ; L_{2}\left(\Gamma_{0}\right)\right) .
$$

But, as noted explicitly in [22, page 122] in the characteristic sector, time regularity is equivalent to (interchangeable with) tangential space regularity (since the corresponding dual variables have comparable bounds from above and below in such sector). Therefore, the statement $\left.\zeta_{t}\right|_{\Sigma_{0}} \in H_{\mathrm{cs}}^{a-1}\left(0, T ; L_{2}\left(\Gamma_{0}\right)\right)$ contained in (3.48) equivalently implies

$$
\left.\zeta_{t}\right|_{\Sigma_{0}} \in H_{\mathrm{cs}}^{0}\left(0, T ; H^{a-1}\left(\Gamma_{0}\right)\right) .
$$


Then, (3.49) combined with $\left.\zeta_{t}\right|_{\Sigma_{0}} \in H_{\mathrm{ncs}}^{0}\left(\Sigma_{0}\right)$ contained in (3.48) ultimately leads (since $a-1<0$ ) to the sought-after estimate

$$
\begin{aligned}
& \left.\zeta_{t}\right|_{\Sigma_{0}} \in L_{2}\left(0, T ; H^{a-1}\left(\Gamma_{0}\right)\right) \text { continuously in }\left\{z_{0}, z_{1}\right\} \in H^{1}(\Omega) \times L_{2}(\Omega): \\
& \qquad \int_{0}^{T}\left\|\zeta_{t}\right\|_{H^{a-1}\left(\Gamma_{0}\right)}^{2} d t \leq C_{T}\left\|\left\{z_{0}, z_{1}\right\}\right\|_{H^{1}(\Omega) \times L_{2}(\Omega)}^{2} .
\end{aligned}
$$

Analysis of w-problem (3.44). Recalling [22, Remark 3.6, page 136, Corollary 4.3, page 148, and Remark, page 121, bottom], [23, Corollary 3.4b, page 113], with $(2 a-1)+(1-a)=a$, we have that, the map

$$
\begin{aligned}
g & \left.\in L_{2}\left(0, T ; H^{1-a}\left(\Gamma_{0}\right)\right) \longrightarrow w\right|_{\Sigma_{0}} \in H^{a}\left(\Sigma_{0}\right) \\
& \equiv H^{a}\left(0, T ; L_{2}\left(\Gamma_{0}\right)\right) \cap L_{2}\left(0, T ; H^{a}\left(\Gamma_{0}\right)\right)
\end{aligned}
$$

is continuous (see detailed justification below). Compare with (3.45) for $\left.\zeta\right|_{\Sigma_{0}}$. Then the very same argument from (3.45) through (3.50) given for $\left.\zeta\right|_{\Sigma_{0}}$ applies now verbatim to $\left.w\right|_{\Sigma_{0}}$, and yields the continuity of the map

$$
\begin{gathered}
\left.g \in L_{2}\left(0, T ; H^{1-a}\left(\Gamma_{0}\right)\right) \longrightarrow w_{t}\right|_{\Sigma_{0}} \in L_{2}\left(0, T ; H^{a-1}\left(\Gamma_{0}\right)\right) ; \\
\int_{0}^{T}\left\|w_{t}\right\|_{H^{a-1}\left(\Gamma_{0}\right)}^{2} d t \leq C_{T} \int_{0}^{T}\|g\|_{H^{1-a}\left(\Gamma_{0}\right)}^{2} d t,
\end{gathered}
$$

as desired. Hence, combining (3.50) with (3.52) for $z=\zeta+w$ in (3.43), we obtain estimate (3.42), as desired.

A more detailed explanation is needed to justify the full statement in (3.51) on the basis of [22, 23]. First, [23, Corollary 3.4, page 148] and [22, Corollary 4.3(b)] yield, indeed, the conclusion of (3.51), but under the seemingly stronger assumption that $g \in H^{1-a}\left(\Sigma_{0}\right) \equiv L_{2}\left(0, T ; H^{1-a}\left(\Gamma_{0}\right)\right) \cap H^{1-a}\left(0, T ; L_{2}\left(\Gamma_{0}\right)\right)$. However, as already noted above, following (3.48), the original assumption $g \in$ $L_{2}\left(0, T ; H^{1-a}\left(\Gamma_{0}\right)\right)$ in (3.51) also yields: (i) $g \in H_{\mathrm{cs}}^{1-a}\left(\Sigma_{0}\right)$ (in the characteristic sector), and hence (a): $\left.w\right|_{\Sigma_{0}} \in H_{\mathrm{cs}}^{a}\left(\Sigma_{0}\right)$ (in the characteristic sector), as recalled above; as well as, a fortiori, (ii) $g \in H_{\mathrm{ncs}}^{0}\left(\Sigma_{0}\right)$ (in the non-characteristic sector), and hence $w \in H_{\mathrm{ncs}}^{1}(Q)$ and (b): $\left.w\right|_{\Sigma_{0}} \in H_{\mathrm{ncs}}^{1}\left(\Sigma_{0}\right)$ (in the non-characteristic sector). Thus, combining (a) and (b) yields the full statement of (3.51), as desired.

Step 2. Equipped with the result of Proposition 3.8, we now follow the same procedure as in Section 3.2 with some appropriate modifications. The counterpart of Lemma 3.1 is now the following.

Lemma 3.9. Assume Hypotheses 1.1 and 1.2 and, in addition, that there exists a constant $\theta_{0}>0$ such that

$$
H^{1-a}\left(\Gamma_{0}\right)=\mathscr{D}\left(\mathscr{A}^{\theta_{0}}\right), \quad \text { satisfying } \alpha-2 r \geq 2 \theta_{0},
$$


as in assumption (1.7a). Then, setting $D_{0}=0$ (hence $\beta=0$ ) in (1.2c) or in (3.2a), the corresponding solution of (1.2) with $z_{t}(0, \cdot)=v(0, \cdot)=0$, or (3.2), satisfies the following estimates for $0 \leq t \leq T$ :

(i)

$$
\begin{array}{r}
\left\|A_{N}^{1 / 2} z(t)\right\|_{L_{2}(\Omega)}^{2}+\left\|z_{t}(t)\right\|_{L_{2}(\Omega)}^{2}+2 d_{2} \int_{0}^{t}\left\|z_{\tau}(\tau)\right\|_{L_{2}\left(\Gamma_{1}\right)}^{2} d \tau \\
\leq C_{T}\left[\int_{0}^{t}\left\|v_{\tau}(\tau)\right\|_{\mathscr{D}\left(A^{\theta_{0}}\right)}^{2} d \tau+\left\|A_{N}^{1 / 2} z_{0}\right\|_{L_{2}(\Omega)}^{2}\right]
\end{array}
$$

(ii)

$$
\begin{aligned}
\int_{0}^{t}\left\|N_{0}^{*} A_{N} z_{\tau}(\tau)\right\|_{\left[\mathscr{D}\left(\mathscr{A}^{\theta_{0}}\right)\right]^{\prime}}^{2} d \tau & \equiv \int_{0}^{t}\left\|z_{\tau}(\tau)\right\|_{H^{a-1}\left(\Gamma_{0}\right)}^{2} d \tau \\
& \leq C_{T}\left[\int_{0}^{t}\left\|v_{\tau}(\tau)\right\|_{\mathscr{D}\left(\mathscr{A}^{\left.\theta_{0}\right)}\right.}^{2} d \tau+\left\|A_{N}^{1 / 2} z_{0}\right\|_{L_{2}(\Omega)}^{2}\right] .
\end{aligned}
$$

Proof. (i) We follow the proof of Lemma 3.1 with appropriate modifications, where now $D_{0}=0$ (and $\beta=0$ ). More precisely, the counterpart of (3.7) is now (we set $d_{2}=0$ just to streamline the relevant formulas, as these are innocuous terms)

$$
\begin{aligned}
& \frac{1}{2} \frac{d}{d t}\left\{\left(z_{t}(t), z_{t}(t)\right)_{L_{2}(\Omega)}+\left(A_{N} z(t), z(t)\right)_{L_{2}(\Omega)}\right\} \\
& =\left(v_{t}(t), N_{0}^{*} A_{N} z_{t}(t)\right)_{L_{2}\left(\Gamma_{0}\right)}=\left(v_{t}(t),\left.z_{t}(t)\right|_{\Gamma_{0}}\right)_{L_{2}\left(\Gamma_{0}\right)} .
\end{aligned}
$$

Integrating (3.56) in time from 0 to $t$ with initial conditions $z(0)=z_{0}, z_{t}(0)=0$ by $(3.2 \mathrm{c})$, we obtain the counterpart of (3.8)

$$
\begin{aligned}
\| z_{t}(t) & \left\|_{L_{2}(\Omega)}^{2}+\right\| A_{N}^{1 / 2} z(t)\left\|_{L_{2}(\Omega)}^{2}-\right\| A_{N}^{1 / 2} z_{0} \|_{L_{2}(\Omega)}^{2} \\
& =2 \int_{0}^{t}\left(v_{\tau}(\tau),\left.z_{\tau}(\tau)\right|_{\Gamma_{0}}\right)_{L_{2}\left(\Gamma_{0}\right)} d t \\
& \leq 2 \int_{0}^{t}\left\|v_{\tau}(\tau)\right\|_{H^{1-a}\left(\Gamma_{0}\right)}\left\|z_{\tau}(\tau)\right\|_{H^{a-1}\left(\Gamma_{0}\right)} d \tau \\
& \leq \int_{0}^{t}\left\|z_{\tau}(\tau)\right\|_{H^{a-1}\left(\Gamma_{0}\right)}^{2} d \tau+\int_{0}^{t}\left\|v_{\tau}(\tau)\right\|_{H^{1-a}\left(\Gamma_{0}\right)}^{2} d \tau .
\end{aligned}
$$

Next, for the first term on the right-hand side of (3.58) we invoke Proposition 3.8 , (3.42) with $g=v_{t}$ (see (1.2c) with $D_{0}=0$ and (3.41d)) to get with $z_{1}=0$, on $0 \leq t \leq T$ :

$$
\int_{0}^{t}\left\|z_{\tau}(\tau)\right\|_{H^{a-1}\left(\Gamma_{0}\right)}^{2} d \tau \leq C_{T}\left[\int_{0}^{t}\left\|v_{\tau}(\tau)\right\|_{H^{1-a}\left(\Gamma_{0}\right)}^{2} d \tau+\left\|A_{N}^{1 / 2} z_{0}\right\|_{L_{2}(\Omega)}^{2}\right] .
$$


Inserting (3.59) into (3.58) yields

$$
\begin{aligned}
& \left\|z_{t}(t)\right\|_{L_{2}(\Omega)}^{2}+\left\|A_{N}^{1 / 2} z(t)\right\|_{L_{2}(\Omega)}^{2} \\
& \quad \leq C_{T}\left[\int_{0}^{t}\left\|v_{\tau}(\tau)\right\|_{H^{1-a}\left(\Gamma_{0}\right)}^{2} d \tau+\left\|A_{N}^{1 / 2} z_{0}\right\|_{L_{2}(\Omega)}^{2}\right] .
\end{aligned}
$$

Finally, using assumption (3.53), $H^{1-a}\left(\Gamma_{0}\right)=\mathscr{D}\left(\mathscr{A}^{\theta_{0}}\right)$, on the right-hand side of (3.60) yields estimate (3.54), as desired.

(ii) We return to (3.59), use again assumption (3.53) including this time with the dual version $H^{a-1}\left(\Gamma_{0}\right)=\left[\mathscr{D}\left(\mathscr{A}^{\theta_{0}}\right)\right]^{\prime}$, and obtain estimate (3.55), as desired.

Step 3. At this point, we need to estimate the integral on the right-hand side of (3.54), (3.55) in terms of $\mathscr{A}^{-r} v_{2} \in L_{2}\left(\Gamma_{0}\right)$. Thus, the counterpart of the critical Lemma 3.3 is now the following.

Lemma 3.10. Assume Hypotheses 1.1 and 1.2 as well as (3.53): $H^{1-a}\left(\Gamma_{0}\right)=\mathscr{D}\left(\mathscr{A}^{\theta_{0}}\right)$ where $\alpha-2 r \geq 2 \theta_{0}$. Then, the component $v_{t}$ of the solution of system (3.2) (or (1.2) with $z_{t}(0)=v(0)=0$ ), with $D_{0}=0$ (hence $\beta=0$ ) satisfies the following estimate on $0 \leq t \leq T$, for constant $C_{2}$ and $C_{3}$ depending on $T$ :

(i)

$$
\int_{0}^{t}\left\|\mathscr{A}^{\theta_{0}} v_{\tau}(\tau)\right\|_{L_{2}\left(\Gamma_{0}\right)}^{2} d \tau \leq C_{3} e^{C_{2} t}\left[\left\|\mathscr{A}^{-r} v_{2}\right\|_{L_{2}\left(\Gamma_{0}\right)}^{2}+\left\|A_{N}^{1 / 2} z_{0}\right\|_{L_{2}(\Omega)}^{2}\right] .
$$

(ii) Consequently, by (3.55) and (3.61), we have

$$
\begin{aligned}
\int_{0}^{t}\left\|N_{0}^{*} A_{N} z_{\tau}(\tau)\right\|_{\left[\mathscr{D}\left(\mathscr{A}^{\theta_{0}}\right)\right]^{\prime}}^{2} d \tau & \equiv \int_{0}^{t}\left\|z_{\tau}(\tau)\right\|_{H^{a-1}\left(\Gamma_{0}\right)}^{2} d \tau \\
& \leq C e^{C t}\left[\left.\left\|\mathscr{A}^{-r} v_{2}\right\|\right|_{L_{2}\left(\Gamma_{0}\right)} ^{2}+\left\|A_{N}^{1 / 2} z_{0}\right\|_{L_{2}(\Omega)}^{2}\right],
\end{aligned}
$$

for a constant $C>0$ depending on $T$.

Proof. (i) We repeat verbatim the proof of Lemma 3.3 (both the one given in Section 3.2, and the one given in Appendix B), with the only difference that the assumption $\alpha-2 r \geq-2 r_{0}$ of Lemma 3.3 is now replaced by the present assumption $\alpha-2 r \geq 2 \theta_{0}$, that is, $\left(-r_{0}\right)$ there is now replaced by $\theta_{0}$. This way, conclusion (3.11) of Lemma 3.3 becomes precisely conclusion (3.61) now.

(ii) We use (3.61) in (3.55) and obtain (3.62).

The remaining part of the proof of Theorem 1.7(II) from Proposition 3.5 to the end of Section 3.2 remains unchanged and produces Theorem 1.7(I).

\section{Spectral/stability analysis of the uncoupled hyperbolic $z$-problem}

4.1. Spectral character of the origin $\lambda=0$ depending upon the strength of the operator $D_{0}$. So far, in achieving the singular estimate (1.8), the term $\beta D_{0} z$ on 
the "flexible wall" $\Gamma_{0}$ in $(1.2 \mathrm{c})$ has played no critical role. In particular, Theorem 1.7 holds true also for the simplest model with $\beta=0$ in $(1.2 \mathrm{c})$, as noted in that theorem. It turns out, however, that if the boundary operator $D_{0}$ required by Theorem 1.7 (via assumption (1.9)) is of sufficiently high unboundedness (see Proposition 4.3 below) as is required in the case $\alpha=1 / 2$, then its impact on the stability properties of the overall system is negative, in the sense that the system then becomes unstable; more precisely, the origin $\lambda=0$ becomes a point of the continuous spectrum of the dynamics operator. To gain enlightenment on this issue, it suffices to consider the uncoupled hyperbolic $z$-problem, the critical component of problem (1.2). Thus, throughout this section we consider the following hyperbolic dynamics with $d_{1}>0$ :

$$
\begin{array}{ll}
z_{t t}=\Delta z-d_{2} z_{t} & \text { in }(0, T] \times \Omega \equiv Q ; \\
\frac{\partial z}{\partial v}+\left.d_{1} z\right|_{\Sigma_{1}}=0 & \text { in }(0, T] \times \Gamma_{1} \equiv \Sigma_{1} \\
{\left[\frac{\partial z}{\partial v}+D_{0} z_{t}+\beta D_{0} z\right]_{\Sigma_{0}}=0} & \text { in }(0, T] \times \Gamma_{0}=\Sigma_{0} ; \\
z(0, \cdot)=z_{0}, \quad z_{t}(0, \cdot)=z_{1} & \text { in } \Omega .
\end{array}
$$

Its abstract model is (specializing (2.7)),

$$
z_{t t}+A_{N} z+\beta A_{N} N_{0} D_{0} N_{0}^{*} A_{N} z+A_{N} N_{0} D_{0} N_{0}^{*} A_{N} z_{t}+d_{2} z_{t}=0
$$

or (by $(2.12 \mathrm{a}))$,

$$
\frac{d}{d t}\left[\begin{array}{c}
z \\
z_{t}
\end{array}\right]=A_{1}\left[\begin{array}{c}
z \\
z_{t}
\end{array}\right]
$$

where, actually, in the present section, $A_{1}$ will denote the realization of the operator

$$
\left[\begin{array}{cc}
0 & I \\
-A_{N}-\beta A_{N} N_{0} D_{0} N_{0}^{*} A_{N} & -A_{N} N_{0} D_{0} N_{0}^{*} A_{N}-d_{2} I
\end{array}\right]
$$

on either the space $\mathbb{E}$ or the space $\mathbb{Z}$ in (4.5), (4.7) below, respectively, and either for $\beta=0$, or else for $\beta=1$ in both cases. These values of $\beta$ are the most representative ones. Thus, in effect, the symbol $A_{1}$ stands for four operators: we will explicitly specify the value of $\beta$, the underlying space, and corresponding domain in question, so no confusion is likely to arise. Regarding the state space selected for $\left\{z, z_{t}\right\}$ and hence the corresponding definition of $\mathscr{D}\left(A_{1}\right)$, we will study the original problem (4.1), or equivalently its abstract version $(4.2),(4.3),(4.4)$ in two frameworks both for $\beta=0$ and $\beta=1$. 
Framework 1 (energy space). Here for $\beta=0$ or $\beta=1$, the state space chosen is the “energy space” $\mathbb{E}$,

$$
\begin{aligned}
\mathbb{E} & \equiv \mathscr{D}\left(A_{N}^{1 / 2}\right) \times L_{2}(\Omega) ; \\
\|f\|_{\mathbb{E}}^{2} & =\left\|A_{N}^{1 / 2} f_{1}\right\|_{L_{2}(\Omega)}^{2}+\left\|f_{2}\right\|_{L_{2}(\Omega)}^{2}, \quad f=\left[f_{1}, f_{2}\right] .
\end{aligned}
$$

Then

(\#1i) for $\beta=0$, the space $\mathbb{E}$ coincides with the space $Y_{1}$ in (2.8) and (2.9) for $\beta=0$; moreover, the operator $A_{1}: \mathbb{E} \supset \mathscr{D}\left(A_{1}\right) \rightarrow \mathbb{E}$ in (4.4), (4.5) with the usual domain given by $(2.12 \mathrm{~b})$ with $\beta=0$ is dissipative, in fact maximal dissipative, and thus the generator of an s.c. contraction semigroup $e^{A_{1} t}$ on $\mathbb{E}$ (this is nothing but Lemma 2.4 for $\beta=0$ );

(\#1ii) for $\beta=1$, the operator $A_{1}: \mathbb{E} \supset \mathscr{D}\left(A_{1}\right) \rightarrow \mathbb{E}$ in (4.4), (4.5), this time with the domain

$$
\begin{aligned}
& \mathscr{D}\left(A_{1}\right)=\left\{\left[h_{1}, h_{2}\right]: h_{1}, h_{2} \in \mathscr{D}\left(A_{N}^{1 / 2}\right),\right. \\
&\left.h_{1}+N_{0} D_{0} N_{0}^{*} A_{N} h_{1}+N_{0} D_{0} N_{0}^{*} A_{N} h_{2} \in \mathscr{D}\left(A_{N}\right)\right\}
\end{aligned}
$$

is not dissipative on $\mathbb{E}$. Nevertheless, we have the following lemma.

Lemma 4.1. Assume Hypotheses 1.1, 1.2, and 1.3, and let $\beta=1$. Then the operator $A_{1}$ in (4.4) with domain as in (4.6) is the generator of an s.c. semigroup $e^{A_{1} t}$ on $\mathbb{E}$ which, moreover, is uniformly bounded on $\mathbb{E}$ (but not contraction).

A proof may be given using a Galerkin approximation argument. One starts with the final dimensional projection on $\mathbb{E}$ of the original problem. For this finite-dimensional approximation, the requires a priori global bounds (uniformly in the parameter of approximation) are obtained by the energy method (multiplication by $z_{t}$ ). This then allows passage to the limit. Details are, however, omitted also because this result is not critical in the present paper.

Framework 2. Here for $\beta=0$ or $\beta=1$, we take as the state space the space $\mathbb{Z}$ defined by

$$
\begin{gathered}
\mathbb{Z}=Z_{1} \times L_{2}(\Omega) ; \\
\left\|f_{1}\right\|_{Z_{1}}^{2}=\left\|A_{N}^{1 / 2} f_{1}\right\|_{L_{2}(\Omega)}^{2}+\left\|D_{0}^{1 / 2} N_{0}^{*} A_{N} f_{1}\right\|_{L_{2}\left(\Gamma_{0}\right)}^{2},
\end{gathered}
$$

where $Z_{1}$ is therefore the space defined in (2.8) and (2.9) for $\beta=1$. Then

(\#2i) for $\beta=1$, the space $\mathbb{Z}$ coincides with $Y_{1}$ in (2.10) for $\beta=1$; moreover, the operator $A_{1}: \mathbb{Z} \supset \mathscr{D}\left(A_{1}\right) \rightarrow \mathbb{Z}$ in (4.4), (4.7), with the usual domain given by $(2.12 \mathrm{~b})$ with $\beta=1$ is dissipative, in fact maximal dissipative, and thus the generator of an s.c. contraction semigroup $e^{A_{1} t}$ on $\mathbb{Z}$ (this is nothing but Lemma 2.4 for $\beta=1$ ); 
(\#2ii) for $\beta=0$, the operator $A_{1}: \mathbb{Z} \supset \mathscr{D}\left(A_{1}\right) \rightarrow \mathbb{Z}$ in (4.4), (4.7) with domain $\mathscr{D}\left(A_{1}\right)$ given by

$$
\mathscr{D}\left(A_{1}\right)=\left\{\left[h_{1}, h_{2}\right]: h_{1}, h_{2} \in \mathbb{Z}, h_{1}+N_{0} D_{0} N_{0}^{*} A_{N} h_{2} \in \mathscr{D}\left(A_{N}\right)\right\}
$$

is not dissipative on $\mathbb{Z}$ (same reason as in case (\#1ii) above). Nevertheless, analogously to Lemma 4.1, we have the following lemma.

Lemma 4.2. Assume Hypotheses 1.1, 1.2, and 1.3, and let $\beta=0$. Then the operator $A_{1}$ in (4.4) with domain (4.8) is the generator of an s.c. semigroup $e^{A_{1} t}$ on $\mathbb{Z}$ which, moreover, is uniformly bounded on $\mathbb{Z}$ (but not contraction).

Spectral consequences. In either framework 1 or 2 , with either $\beta=0$ or else $\beta=1$, $A_{1}$ with the appropriate domain generates an s.c. uniformly bounded semigroup on $\mathbb{E}$, respectively, $\mathbb{Z}$. Therefore, the open right half-plane belongs to the resolvent $\rho\left(A_{1}\right)$ of $A_{1}$ in either case:

$$
\{\lambda: \operatorname{Re} \lambda>0\} \in \rho\left(A_{1}\right) \text {, either } \beta=0 \text { or } \beta=1 \text {; either on } \mathbb{E} \text {, or on } \mathbb{Z} \text {. }
$$

In the present section, combined with the more substantial Section 6 below, we describe the spectral situation for the operator $A_{1}$ on the imaginary axis. More precisely, the next proposition is enlightening.

Orientation on Proposition 4.3. The significance of Proposition 4.3 may be stated as follows.

(i) If the boundary operator $D_{0}$ is mild, of order only up to 1 , then we may take $\beta=0$ in problem (4.1) (simplest model, most desirable situation), and still obtain that the origin is in the resolvent set $\rho\left(A_{1}\right)$ of $A_{1}, 0 \in \rho\left(A_{1}\right)$, for $A_{1}$ realized either on the space $\mathbb{E}$ or on the space $\mathbb{Z}$.

(ii) If, instead, the boundary operator $D_{0}$ is stronger, of order $s, 1<s \leq 2$, as needed in the case $\alpha=1 / 2$ of Theorem 1.7, then

(a) taking $\beta=0$ on problem (4.1) (simplest model) has the negative implication that the origin belongs to the continuous spectrum of $A_{1}$, $0 \in \sigma_{c}\left(A_{1}\right)$, for $A_{1}$ realized either on the space $\mathbb{E}$ or on the space $\mathbb{Z}$; and so, problem (4.1) is accordingly unstable in both these cases;

(b) in order to obtain, again, that $0 \in \rho\left(A_{1}\right)$, both on $\mathbb{E}$ or on $\mathbb{Z}$, it is necessary to set $\beta=1$ (or $\beta \neq 0)$ in problem (4.1).

A restatement from an alternative angle is the following. In its first part, Proposition 4.3 says that if $\beta=0$ (the simplest, most desirable situation for problem (4.1) to enjoy the singular estimate (1.8)), the origin belongs to the continuous spectrum of the operator $A_{1}, 0 \in \sigma_{c}\left(A_{1}\right)$, whether $A_{1}$ is viewed on $\mathbb{E}$ or on $\mathbb{Z}$, except for the less interesting case where $D_{0}$ is at most a first-order (rather than second-order, as needed for $\alpha=1 / 2$ ) differential operator on $\Gamma_{0}$. In this latter case then, the origin is in the resolvent set of $A_{1}, 0 \in \rho\left(A_{1}\right)$, whether $A_{1}$ is viewed on $\mathbb{E}$ or on $\mathbb{Z}$. 
Moreover, in its second part, Proposition 4.3 says that the insertion of the boundary term $D_{0} z$ on $\Gamma_{0}$ in (4.1c) $(\beta=1)$, or the term $D_{0} N_{0}^{*} A_{N} z$ in $(4.2)(\beta=1)$ has the effect of removing the origin from the (continuous) spectrum of $A_{1}$ in the pathological case (4.12) of Proposition 4.3(i) with $D_{0}$ at least of order 1 , so that with $\beta=1$, we have that $0 \in \rho\left(A_{1}\right)$ both on $\mathbb{E}$ and on $\mathbb{Z}$, even with $D_{0}$ a second-order tangential operator (as in Assumption 1.3).

Proposition 4.3. Assume Hypothesis 1.1, and, moreover, that $D_{0}$ is a positive, selfadjoint operator on $L_{2}\left(\Gamma_{0}\right)$, of order s so that

$$
D_{0}: \text { continuous } H^{p}\left(\Gamma_{0}\right) \longrightarrow H^{p-s}\left(\Gamma_{0}\right)
$$

(i) Let $\beta=0$ in (4.4) or (4.1c). Then

$$
\begin{aligned}
& \text { if } 0 \leq s \leq 1 \text {, then } \Longrightarrow 0 \in \rho\left(A_{1}\right)=\text { resolvent set of } A_{1} \text {, } \\
& \text { if } 1<s \leq 2 \text {, then } \Longrightarrow 0 \in \sigma_{c}\left(A_{1}\right)=\text { continuous spectrum of } A_{1} \text {, }
\end{aligned}
$$

regardless of whether $A_{1}$ is defined on the space $\mathbb{E}$ in (4.5), or on the space $\mathbb{Z}$ in (4.7).

(ii) Let $\beta=1$, and let $1<s \leq 2$. Then,

$$
0 \in \rho\left(A_{1}\right)
$$

regardless of whether $A_{1}$ is defined on the space $\mathbb{E}$, or on the space $\mathbb{Z}$; thus removing the pathology in part (i), (4.12).

Proof. (i) For $\beta=0$, we will see that the operator $A_{1}$ is injective; and that its inverse $A_{1}^{-1}$ is a bounded operator on $\mathbb{E}$, or on $\mathbb{Z}$, if $0 \leq s \leq 1$; and is not a bounded operator on $\mathbb{E}$, or on $\mathbb{Z}$, if $1<s \leq 2$. Let $\left[f_{1}, f_{2}\right]$ be given with $f_{2} \in L_{2}(\Omega)$ and with $f_{1}$ either in $\mathscr{D}\left(A_{N}^{1 / 2}\right) \equiv H^{1}(\Omega)$, or else in $Z_{1}$ (defined by $(4.7)$ ). With $\beta=0$, we seek to solve

$$
\begin{aligned}
& A_{1}\left[\begin{array}{l}
z_{1} \\
z_{2}
\end{array}\right]=\left[\begin{array}{l}
f_{1} \\
f_{2}
\end{array}\right], \text { or } \\
& \left\{\begin{array}{l}
z_{2}=f_{1}, \\
-A_{N}\left[z_{1}+N_{0} D_{0} N_{0}^{*} A_{N} z_{2}\right]-d_{2} z_{2}=f_{2}
\end{array}\right.
\end{aligned}
$$

for $\left[z_{1}, z_{2}\right] \in \mathscr{D}\left(A_{1}\right)$, on either the space $\mathbb{E}$ or $\mathbb{Z}$ (see (2.12b) and (4.8), respectively, for $\left.\mathscr{D}\left(A_{1}\right)\right)$. Injectivity of $A_{1}$ follows at once. $\left(A_{N}\right.$ is injective, in fact boundedly invertible, on $L_{2}(\Omega)$, see (2.1) with $d_{1}>0$.) Moreover, solving (4.14) one has

$$
-z_{1}=A_{N}^{-1}\left(d_{2} f_{1}+f_{2}\right)+N_{0} D_{0} N_{0}^{*} A_{N} f_{1}
$$


Let $f_{1}$ be either in $\mathscr{D}\left(A_{N}^{1 / 2}\right)$, or else in $Z_{1}$. It remains to test whether $\left[z_{1}, z_{2}\right] \in$ $\mathscr{D}\left(A_{1}\right)$. We have that

$$
\begin{array}{ll}
N_{0}^{*} A_{N} f_{1} \in H^{1 / 2}\left(\Gamma_{0}\right), & \text { if } f_{1} \in \mathscr{D}\left(A_{N}^{1 / 2}\right) \equiv H^{1}(\Omega), \\
D_{0}^{1 / 2} N_{0}^{*} A_{N} f_{1} \in L_{2}\left(\Gamma_{0}\right), & \text { if } f_{1} \in Z_{1} .
\end{array}
$$

In fact, (4.16a) follows by (2.6) on $N_{0}^{*} A_{N}$ and trace theory; while, (4.16b) follows by definition (4.7b) of $Z_{1}$. Next, we need to distinguish between the two cases assumed on $D_{0}$.

First, let $0 \leq s \leq 1$. It then follows from (4.10) for $p=1 / 2$ and (4.16a) that $D_{0} N_{0}^{*} A_{N} f_{1} \in H^{-1 / 2}\left(\Gamma_{0}\right)$ in both cases $f_{1} \in \mathscr{D}\left(A_{N}^{1 / 2}\right)$ and $f_{1} \in Z_{1}$. Then, by (2.5), it follows that (\#): $N_{0} D_{0} N_{0}^{*} A_{N} f_{1} \in H^{1}(\Omega) \equiv \mathscr{D}\left(A_{N}^{1 / 2}\right)$; and then by (4.15), $z_{1} \in$ $\mathscr{D}\left(A_{N}^{1 / 2}\right)$ in both cases, as desired. Thus, $(4.11)$ is proved on $\mathbb{E}$. Moreover, if $f_{1} \in$ $Z_{1}$, it follows from (4.15) that

$$
\begin{aligned}
-D_{0}^{1 / 2} N_{0}^{*} A_{N} z_{1}= & D_{0}^{1 / 2} N_{0}^{*} A_{N} A_{N}^{-1}\left(d_{2} f_{1}+f_{2}\right) \\
& +D_{0}^{1 / 2} N_{0}^{*} A_{N}\left(N_{0} D_{0} N_{0}^{*} A_{N} f_{1}\right) \in L_{2}\left(\Gamma_{0}\right) .
\end{aligned}
$$

To justify the membership in (4.17), we recall (2.6), trace theory, (4.10) on $D_{0}$ with $0 \leq s \leq 1$, and (\#) a few lines above for the second term in (4.17). Thus $z_{1} \in Z_{1}$ as well, and (4.11) is proved also for $\mathbb{Z}$.

Let now $1<s \leq 2$ in (4.10). Then the same argument employed above shows that $z_{1} \notin H^{1}(\Omega)$ for $f_{1} \in H^{1}(\Omega)$, as $D_{0}^{1 / 2}$ loses $1 / 2+\epsilon$ and $N_{0}$ gains $3 / 2$. Thus, $N_{0} D_{0} N_{0}^{*} A_{N} f_{1} \in H^{1-\epsilon}(\Omega)$ in both cases, $f_{1} \in H^{1}(\Omega)$ and $f_{1} \in Z_{1}$. Hence, (4.12) is proved.

(ii) Let now $\beta=1$. Let $\left[f_{1}, f_{2}\right]$ be given with $f_{2} \in L_{2}(\Omega)$, and with $f_{1}$ either in $\mathscr{D}\left(A_{N}^{1 / 2}\right)$, or else in $Z_{1}$. We seek accordingly to solve, with $\beta=1$,

$$
\begin{aligned}
& A_{1}\left[\begin{array}{l}
z_{1} \\
z_{2}
\end{array}\right]=\left[\begin{array}{l}
f_{1} \\
f_{2}
\end{array}\right], \text { or } \\
& \left\{\begin{array}{l}
z_{2}=f_{1} \\
-A_{N}\left\{\left[I+N_{0} D_{0} N_{0}^{*} A_{N}\right] z_{1}+N_{0} D_{0} N_{0}^{*} A_{N} z_{2}\right\}-d_{2} z_{2}=f_{2},
\end{array}\right.
\end{aligned}
$$

for $\left[z_{1}, z_{2}\right] \in \mathscr{D}\left(A_{1}\right)$, on either space $\mathbb{E}$ or $\mathbb{Z}$ (see (4.6) and (2.12a) for $\mathscr{D}\left(A_{1}\right)$, respectively). Solving (4.18) yields

$$
\begin{aligned}
-[I+S] A_{N}^{1 / 2} z_{1} & =-\left[I+A_{N}^{1 / 2} N_{0} D_{0} N_{0}^{*} A_{N}^{1 / 2}\right] A_{N}^{1 / 2} z_{1} \\
& =A_{N}^{-1 / 2}\left(d_{2} f_{1}+f_{2}\right)+A_{N}^{1 / 2} N_{0} D_{0} N_{0}^{*} A_{N} f_{1} \\
& =S A_{N}^{1 / 2} f_{1}+\psi,
\end{aligned}
$$


where $\psi=A_{N}^{-1 / 2}\left(d_{2} f_{1}+f_{2}\right) \in \mathscr{D}\left(A_{N}^{1 / 2}\right)$, and

$$
\begin{gathered}
S \equiv A_{N}^{1 / 2} N_{0} D_{0} N_{0}^{*} A_{N}^{1 / 2} \\
S A_{N}^{1 / 2} f_{1}+\psi \in \begin{cases}{\left[\mathscr{D}\left(A_{N}^{1 / 2}\right)\right]^{\prime}} & \text { if } f_{1} \in \mathscr{D}\left(A_{N}^{1 / 2}\right), \\
{\left[\mathscr{D}\left(A_{N}^{1 / 4}\right)\right]^{\prime}} & \text { if } f_{1} \in Z_{1} .\end{cases}
\end{gathered}
$$

To justify the membership in (4.20), let $f_{1} \in \mathscr{D}\left(A_{N}^{1 / 2}\right)$ (resp., let $\left.f_{1} \in Z_{1}\right)$ and assume the worst case $s=2$. Then, $N_{0}^{*} A_{N} f_{1} \in H^{1 / 2}\left(\Gamma_{0}\right)$ by (2.6) and trace theory; hence, $D_{0} N_{0}^{*} A_{N} f_{1} \in H^{-3 / 2}\left(\Gamma_{0}\right)$ by (4.10) with $s=2, p=1 / 2$; then $N_{0} D_{0} N_{0}^{*} A_{N} f_{1} \in$ $L_{2}(\Omega)$ by (2.5) (resp., $D_{0}^{1 / 2} N_{0}^{*} A_{N} f_{1} \in L_{2}\left(\Gamma_{0}\right), D_{0} N_{0}^{*} A_{N} f_{1} \in H^{-1}\left(\Gamma_{0}\right), N_{0} D_{0} N_{0}^{*} A_{N} f_{1}$ $\left.\in H^{1 / 2}(\Omega)=\mathscr{D}\left(A_{N}^{1 / 4}\right)\right)$, and then (4.20) follow. We have shown therefore that

$$
S A_{N}^{1 / 2}=A_{N}^{1 / 2} N_{0} D_{0} N_{0}^{*} A_{N} \text { : continuous }\left\{\begin{array}{l}
\mathscr{D}\left(A_{N}^{1 / 2}\right) \longrightarrow\left[\mathscr{D}\left(A_{N}^{1 / 2}\right)\right]^{\prime}, \\
Z_{1} \longrightarrow\left[\mathscr{D}\left(A_{N}^{1 / 4}\right)\right]^{\prime} .
\end{array}\right.
$$

Thus, it follows that the operator $[I+S]$, which is positive selfadjoint on the space $L_{2}(\Omega)$, also satisfies

$$
[I+S]=I+A_{N}^{1 / 2} N_{0} D_{0} N_{0}^{*} A_{N}^{1 / 2}: \text { continuous, injective } L_{2}(\Omega) \longrightarrow\left[\mathscr{D}\left(A_{N}^{1 / 2}\right)\right]^{\prime}
$$

hence, $[I+S]$ is invertible on its range $S\left(L_{2}(\Omega)\right)$. Returning to (4.19) and using this latter information, we find

$$
-A_{N}^{1 / 2} z_{1}=[I+S]^{-1} S A_{N}^{1 / 2} f_{1}+[I+S]^{-1} A_{N}^{-1 / 2}\left(d_{2} f_{1}+f_{2}\right) \in L_{2}(\Omega) .
$$

In (4.23) we have that $A_{N}^{1 / 2} f_{1} \in L_{2}(\Omega)$, while

$$
[I+S]^{-1} S \text { is a bounded operator on } L_{2}(\Omega) \text {, }
$$

by the closed graph theorem [15, page 164] via (4.22). Thus, the membership in (4.23) follows. Then (4.23) shows that

$$
z_{1} \in \mathscr{D}\left(A_{N}^{1 / 2}\right) \text { continuously in }\left[f_{1}, f_{2}\right] \in \mathbb{E} \text {, }
$$

while $z_{2}=f_{1} \in \mathscr{D}\left(A_{N}^{1 / 2}\right)$, so that $0 \in \rho\left(A_{1}\right)$ as desired in (4.13) at least for $A_{1}$ defined on $\mathbb{E}$, with domain given by (4.6). Moreover, from (4.19), taking the inner product with $A_{N}^{1 / 2} z_{1}$ yields

$$
\begin{aligned}
& \left\|A_{N}^{1 / 2} z_{1}\right\|_{L_{2}(\Omega)}^{2}+\left\|D_{0}^{1 / 2} N_{0}^{*} A_{N} z_{1}\right\|_{L_{2}\left(\Gamma_{0}\right)}^{2} \\
& \quad=-\left(D_{0}^{1 / 2} N_{0}^{*} A_{N} f_{1}, D_{0}^{1 / 2} N_{0}^{*} A_{N} z_{1}\right)-\left(d_{2} f_{1}+f_{2}, z_{1}\right),
\end{aligned}
$$

from which it readily follows that if actually $f_{1} \in Z_{1}$, then $z_{1} \in Z_{1}$ as well. Thus, (4.13) is proved also for $A_{1}$ on $\mathbb{Z}$. 
Regarding the remaining points on the imaginary axis, $i \omega, \omega \in \mathbb{R}, \omega \neq 0$, we refer to the forthcoming Section 6: this shows that $i \omega \in \rho\left(A_{1}\right)$ for $\omega \neq 0$.

Remark 4.4. The exact counterpart result of Proposition 4.3 holds true for the overall system (1.1); that is, for the operator $A$ in (2.37) and (2.38) in place of $A_{1}$.

Indeed, writing $A\left[z_{1}, z_{2}, z_{3}, z_{4}, z_{5}\right]=\left[f_{1}, f_{2}, f_{3}, f_{4}, f_{5}\right]$, we now obtain

$$
\begin{gathered}
z_{2}=f_{1}, \\
-A_{N}\left[z_{1}+N_{0} D_{0} N_{0}^{*} A_{N} z_{2}-N_{0} v_{2}\right]-d_{2} z_{2}+A_{N} N_{0} v_{2}=f_{2}, \\
v_{2}=f_{3}, \\
-N_{0}^{*} A_{N} z_{2}-\mathscr{A} v_{1}-\rho \mathscr{A}^{\alpha} v_{2}=f_{4},
\end{gathered}
$$

in place of (4.14); hence

$$
\begin{aligned}
& -z_{1}=A_{N}^{-1}\left(d_{2} f_{1}+f_{2}\right)+N_{0} D_{0} N_{0}^{*} A_{N} f_{1}-N_{0} f_{3}, \\
& -v_{1}=\mathscr{A}^{-1}\left[N_{0}^{*} A_{N} f_{1}+\rho \mathscr{A}^{\alpha} f_{3}+f_{4}\right],
\end{aligned}
$$

in place of (4.15). The new term $N_{0} f_{3}=N_{0}^{*} A_{N} A_{N}^{-1} f_{3}$ in (4.28a) and the new term (4.28b) over the case of Proposition 4.3 are "innocuous." Thus, the analysis in the proof of Proposition 4.3 is the critical one.

4.2. Eigenvalue/vector problem for $A_{1}$ with $\beta=0$ on $\mathbb{E}$ or $\mathbb{Z}$. In this section, we set $\beta=0$ and study the corresponding eigenvalue/vector problem for the operator $A_{1}$ in (4.4) with $\beta=0$ on either the space $\mathbb{E}$, or else the space $\mathbb{Z}$, that is,

$$
\begin{aligned}
& A_{1}\left[\begin{array}{l}
\phi_{1 n} \\
\phi_{2 n}
\end{array}\right]=\lambda_{n}\left[\begin{array}{l}
\phi_{1 n} \\
\phi_{2 n}
\end{array}\right], \text { or } \\
& \left\{\begin{array}{l}
\phi_{2 n}=\lambda_{n} \phi_{1 n}, \\
A_{N}\left[\phi_{1 n}+N_{0} D_{0} N_{0}^{*} A_{N} \phi_{2 n}\right]+\left(\lambda_{n}+d_{2}\right) \phi_{2 n}=0,
\end{array}\right.
\end{aligned}
$$

where $\left\{\phi_{1 n}, \phi_{2 n}\right\}$ are considered either on the space $\mathbb{E}$, and thus normalized as

$$
\left\|A_{N}^{1 / 2} \phi_{1 n}\right\|^{2}+\left\|\phi_{2 n}\right\|^{2} \equiv 1,
$$

or else on the space $\mathbb{Z}$, and hence normalized as

$$
\left\|A_{N}^{1 / 2} \phi_{1 n}\right\|^{2}+\left\|D_{0}^{1 / 2} N_{0}^{*} A_{N} \phi_{1 n}\right\|^{2}+\left\|\phi_{2 n}\right\|^{2} \equiv 1 .
$$

We must have both $\phi_{1 n} \neq 0$ and $\phi_{2 n} \neq 0$ and $\lambda_{n} \neq 0$.

Proposition 4.5. Let $\lambda_{n}$ be an eigenvalue of problem (4.29), (4.30) on $\mathbb{E}$. Then,

(i) if $\operatorname{Im} \lambda_{n} \neq 0$, then

$$
\operatorname{Re} \lambda_{n} \leq-\frac{d_{2}}{2}
$$


212 Singular estimates and uniform stability

(ii) if $\operatorname{Im} \lambda_{n}=0$, then

$$
\begin{gathered}
\operatorname{Re} \lambda_{n}=\lambda_{n}=-\frac{1}{\left\|D_{0}^{1 / 2} N_{0}^{*} A_{N} \phi_{1 n}\right\|^{2}+d_{2}\left\|\phi_{1 n}\right\|^{2}} \\
\left\|D_{0}^{1 / 2} N_{0}^{*} A_{N} \phi_{1 n}\right\|\left\|\phi_{2 n}\right\| \leq \frac{1}{2 \sqrt{d_{2}}} .
\end{gathered}
$$

Thus, if $\left\{\lambda_{n}\right\}$ is a sequence with $\operatorname{Im} \lambda_{n} \equiv 0$, then

$$
\begin{aligned}
\operatorname{Re} \lambda_{n} \nearrow 0 & \Longleftrightarrow\left\|D_{0}^{1 / 2} N_{0}^{*} A_{N} \phi_{1 n}\right\| \longrightarrow \infty \\
& \Longleftrightarrow\left\|\phi_{2 n}\right\| \longrightarrow 0 \Longleftrightarrow\left\|A_{N}^{1 / 2} \phi_{1 n}\right\| \longrightarrow 1 ;
\end{aligned}
$$

(iii) finally, if $D_{0}$ is of order up to 1 (or $D_{0}^{1 / 2}$ is of order up to $1 / 2$ ), then

$$
\begin{aligned}
\left\|D_{0}^{1 / 2} N_{0}^{*} A_{N} \phi_{1 n}\right\| & \leq c\left\|N_{0}^{*} A_{N} \phi_{1 n}\right\|_{H^{1 / 2}\left(\Gamma_{0}\right)} \\
& \leq c\left\|\phi_{1 n}\right\|_{H^{1}(\Omega)} \leq c\left\|A_{N}^{1 / 2} \phi_{1 n}\right\| \leq k,
\end{aligned}
$$

and then (4.35) cannot hold true. More precisely, in this case where $D_{0}$ is of order up to 1 , if $\operatorname{Im} \lambda_{n} \equiv 0$, then

$$
\left|\operatorname{Re} \lambda_{n}\right|=\left|\lambda_{n}\right| \geq \frac{1}{k^{2}+d_{2}\left\|A_{N}^{-1 / 2}\right\|^{2}},
$$

where $k$ is the constant in (4.36).

Proof. Taking the inner product of the second equation in (4.29) with $\phi_{2 n}$ yields

$$
\left(A_{N}^{1 / 2} \phi_{1 n}, A_{N}^{1 / 2} \phi_{2 n}\right)+\left\|D_{0}^{1 / 2} N_{0}^{*} A_{N} \phi_{2 n}\right\|^{2}+\left(\lambda_{n}+d_{2}\right)\left\|\phi_{2 n}\right\|^{2}=0,
$$

and substituting $\phi_{2 n}=\lambda_{n} \phi_{1 n}$ into the first two terms of (4.38) yields

$$
\bar{\lambda}_{n}\left\|A_{N}^{1 / 2} \phi_{1 n}\right\|^{2}+\left|\lambda_{n}\right|^{2}\left\|D_{0}^{1 / 2} N_{0}^{*} A_{N} \phi_{1 n}\right\|^{2}+\left(\lambda_{n}+d_{2}\right)\left\|\phi_{2 n}\right\|^{2} \equiv 0 .
$$

Taking the real part and imaginary part of (4.39) yields

$$
\begin{gathered}
\left(\operatorname{Re} \lambda_{n}\right)\left[\left\|A_{N}^{1 / 2} \phi_{1 n}\right\|^{2}+\right. \\
\left.\left\|\phi_{2 n}\right\|^{2}\right]+d_{2}\left\|\phi_{2 n}\right\|^{2}+\left|\lambda_{n}\right|^{2}\left\|D_{0}^{1 / 2} N_{0}^{*} A_{N} \phi_{1 n}\right\|^{2}=0 \\
\operatorname{Im} \lambda_{n}\left[\left\|\phi_{2 n}\right\|^{2}-\left\|A_{N}^{1 / 2} \phi_{1 n}\right\|^{2}\right]=0
\end{gathered}
$$

Using the normalization (4.30) on $\mathbb{E}$ in (4.40) yields

$$
\left|\lambda_{n}\right|^{2}\left\|D_{0}^{1 / 2} N_{0}^{*} A_{N} \phi_{1 n}\right\|^{2}+\operatorname{Re} \lambda_{n}+d_{2}\left\|\phi_{2 n}\right\|^{2}=0 .
$$

Notice that (4.42) implies $\operatorname{Re} \lambda_{n}<0$, since $\phi_{2 n} \neq 0$.

(i) Let $\operatorname{Im} \lambda_{n} \neq 0$. Then, $\left\|A_{N}^{1 / 2} \phi_{1 n}\right\|^{2}=\left\|\phi_{2 n}\right\|^{2}=1 / 2$ by (4.41) and (4.30). Then, using this information in (4.42) yields $\left(2 \operatorname{Re} \lambda_{n}+d_{2}\right) \leq 0$ and (4.32) is established. 
(ii) Let $\operatorname{Im} \lambda_{n}=0$. Then, (4.42) with $\phi_{2 n}=\lambda_{n} \phi_{1 n}$ yields

$$
\lambda_{n}^{2}\left\|D_{0}^{1 / 2} N_{0}^{*} A_{N} \phi_{1 n}\right\|^{2}+\lambda_{n}+d_{2} \lambda_{n}^{2}\left\|\phi_{1 n}\right\|^{2}=0, \quad \lambda_{n}=\operatorname{Re} \lambda_{n} \neq 0,
$$

which, in turn, yields (4.33). Moreover, (4.36) is readily verified to hold true, by (2.6) and trace theory with $D_{0}^{1 / 2}$ of order up to $1 / 2$. Using (4.30) and (4.36) in (4.33), we obtain (4.37). Finally, solving the quadratic equation (4.42) for real, negative solutions $\lambda_{n}=\operatorname{Re} \lambda_{n}$, imposes that its discriminant be nonnegative, and this condition is precisely (4.34). Then (4.35) follows from (4.30), (4.33), and (4.34).

Proposition 4.6. Let $\lambda_{n}$ be an eigenvalue of problem (4.29), (4.31), on $\mathbb{Z}$. Then,

(i) if $\operatorname{Im} \lambda_{n} \neq 0$, then $\operatorname{Re} \lambda_{n}$ satisfies (4.32);

(ii) if $\operatorname{Im} \lambda_{n}=0$, then

$$
\operatorname{Re} \lambda_{n}=\lambda_{n}=\frac{\left\|D_{0}^{1 / 2} N_{0}^{*} A_{N} \phi_{1 n}\right\|-1}{\left\|D_{0}^{1 / 2} N_{0}^{*} A_{N} \phi_{1 n}\right\|^{2}+d_{2}\left\|\phi_{1 n}\right\|^{2}} .
$$

Thus, if $\left\{\lambda_{n}\right\}$ is a sequence with $\operatorname{Im} \lambda_{n} \equiv 0$, then

$$
\begin{aligned}
\operatorname{Re} \lambda_{n} \nearrow 0 & \Longleftrightarrow\left\|D_{0}^{1 / 2} N_{0}^{*} A_{N} \phi_{1 n}\right\| \longrightarrow 1 \\
& \Longleftrightarrow\left\|A_{N}^{1 / 2} \phi_{1 n}\right\|^{2}+\left\|\phi_{2 n}\right\|^{2} \longrightarrow 0 ;
\end{aligned}
$$

(iii) finally, if $D_{0}$ is of order up to 1 (or $D_{0}^{1 / 2}$ is of order up to $1 / 2$ ), then (4.45) cannot hold true.

Proof. Equations (4.40) and (4.41) are still valid. Thus, if $\operatorname{Im} \lambda_{n} \neq 0$, we now get that $\left\|A_{N}^{1 / 2} \phi_{1 n}\right\|^{2}=\left\|\phi_{2 n}\right\|^{2} \leq 1 / 2$ by (4.41) and (4.31). Using this information in (4.40) yields (4.42) with " $=0$ " replaced by " $\leq 0$ " and hence again (4.32).

Let now $\operatorname{Im} \lambda_{n}=0$. Then, using this time the normalization (4.31) in (4.40) yields now for $\operatorname{Re} \lambda_{n}=\lambda_{n}$ :

$$
\lambda_{n}+d_{2} \lambda_{n}^{2}\left\|\phi_{1 n}\right\|^{2}+\left(\lambda_{n}^{2}-\lambda_{n}\right)\left\|D_{0}^{1 / 2} N_{0}^{*} A_{N} \phi_{1 n}\right\|^{2}=0
$$

in place of (4.43), $\lambda_{n} \neq 0$, from which (4.44) is obtained. Moreover, (4.45a) follows from (4.44) since the denominator of (4.44) is uniformly bounded. Then (4.45a) is equivalent to (4.45b) by (4.31). Finally, (4.45) cannot happen when $D_{0}$ is of order up to 1 by (4.36).

\section{Proof of stability Theorem 1.13 by energy methods in $t$}

Statement of the problem and preliminaries. In this section, we show the uniform stability of the uncontrolled, unforced coupled PDE system (1.2), namely with 
214 Singular estimates and uniform stability

$f \equiv 0$ and $u \equiv 0$. Thus, we will consider the initial boundary value problem

$$
\begin{array}{ll}
z_{t t}=c^{2} \Delta z-d_{2} z_{t}, \quad d_{2}>0, & \text { in }(0, T] \times \Omega ; \\
\frac{\partial}{\partial v} z+d_{1} z=0 & \text { on }(0, T] \times \Gamma_{1} \\
\frac{\partial}{\partial v} z+D_{0} z_{t}+\beta D_{0} z=v_{t} & \text { on }(0, T] \times \Gamma_{0} \\
v_{t t}+A v+\rho \mathscr{A}^{\alpha} v_{t}+\left.\rho_{1} z_{t}\right|_{\Gamma_{0}}=0 & \text { on }(0, T] \times \Gamma_{0} ; \\
{\left[z(\cdot, 0), z_{t}(\cdot, 0), v(\cdot, 0), v_{t}(\cdot, 0)\right]=\left[z_{0}, z_{1}, v_{0}, v_{1}\right]} & \text { in } \Omega \times \Gamma_{0} .
\end{array}
$$

Equivalently, after the innocuous normalization $c=1, \rho_{1}=1$, we rewrite problem (5.1) in abstract form as in (2.7a)-(2.7b) with $f \equiv 0, u \equiv 0$ :

$$
\begin{gathered}
z_{t t}+A_{N} z+\beta A_{N} N_{0} D_{0} N_{0}^{*} A_{N} z+A_{N} N_{0} D_{0} N_{0}^{*} A_{N} z_{t}+d_{2} z_{t}-A_{N} N_{0} v_{t}=0, \\
v_{t t}+\mathscr{A} v+\rho \mathscr{A}^{\alpha} v_{t}+N_{0}^{*} A_{N} z_{t}=0, \\
z(0)=z_{0}, \quad z_{t}(0)=z_{1} ; \quad v(0)=v_{0}, \quad v_{t}(0)=v_{1} .
\end{gathered}
$$

In line with the well-posedness Theorem 1.6, the total energy of the system is given by the square of the $Y_{\beta}$-norm of the solution, with $Y_{\beta} \equiv Z_{\beta} \times L_{2}(\Omega) \times$ $\mathscr{D}\left(\mathscr{A}^{1 / 2}\right) \times L_{2}\left(\Gamma_{0}\right)$ by $(2.11)$ :

$$
\begin{aligned}
E_{\beta}(t) & \equiv\left\|e^{A t} y_{0}\right\|_{Y}^{2} \equiv E_{z, \beta}(t)+E_{v}(t) ; \\
E_{z, \beta}(t) & \equiv\left\|A_{N}^{1 / 2} z(t)\right\|^{2}+\beta\left\|D_{0}^{1 / 2} N_{0}^{*} A_{N} z(t)\right\|^{2}+\left\|z_{t}(t)\right\|^{2} ; \\
E_{v}(t) & \equiv\left\|\mathscr{A}^{1 / 2} v(t)\right\|^{2}+\left\|v_{t}(t)\right\|^{2}, \quad y_{0}=\left[z_{0}, z_{1}, v_{0}, v_{1}\right] .
\end{aligned}
$$

In this section, as in the statement of Theorem 1.13, we emphasize the dependence on $\beta$ explicitly with a subscript.

We recall the hypotheses of Theorem 1.13: in addition to Hypotheses 1.1, 1.2, and 1.3, we assume that $d_{2}>0$, while $d_{1}>0$, (see below (2.1)), and, moreover, that:

Case 1. $\beta=0$ when $D_{0}$ is of order $s, 0 \leq s \leq 1$.

Case 2. $\beta>0$ when $D_{0}$ is of order $s, 1<s \leq 2$.

We then seek to prove the exponential decay (1.21).

Proof of Theorem 1.13. We provide a proof of Theorem 1.13 by energy methods in $t$. In Section 6, we will provide a second proof by using a resolvent characterization (which, in fact, gives more information). The present energy method proof consists in combining the use of standard multipliers $z_{t}$ and $z$ on the $z$ wave equation, with standard multipliers $v_{t}$ and $v$ on the $v$-plate equation.

Our main goal is to prove that, under present assumptions, the following statement holds true: for all $y_{0}=\left[z_{0}, z_{1}, v_{0}, v_{1}\right] \in Y_{\beta}$ there exists a constant $C_{\beta}$, 
which does not depend on $T$, such that

$$
\int_{0}^{T}\left\|e^{A_{\beta} t} y_{0}\right\|_{Y_{\beta}}^{2} d t \equiv \int_{0}^{T} E_{\beta}(t) d t \leq C_{\beta}\left\|y_{0}\right\|_{Y_{\beta}}^{2}=C_{\beta} E_{\beta}(0),
$$

where $Y_{\beta=0} \equiv \mathscr{D}\left(A_{N}^{1 / 2}\right) \times L_{2}(\Omega) \times \mathscr{D}\left(\mathscr{A}^{1 / 2}\right) \times L_{2}\left(\Gamma_{0}\right)$ for $\beta=0$ and $Y_{\beta} \equiv Z_{\beta} \times L_{2}(\Omega)$ $\times \mathscr{D}\left(\mathscr{A}^{1 / 2}\right) \times L_{2}\left(\Gamma_{0}\right)$ for $\beta>0$ (see Theorem 1.13, (2.8), and (2.9)). This is done, as usual, by taking initial conditions, at first, in $\mathscr{D}(A)$ as to justify the computations leading to estimate (5.4) (see, e.g., Remark 2.6), and then extending (5.4) to all $Y$ by density.

Once (5.4) is established, we let $T \nearrow \infty$, invoke Datko's standard theorem [32, page 116] and conclude that the exponential decay in (1.21) holds true: there exist $M_{\beta} \geq 1$ and $\omega_{\beta}>0$ such that $\left\|e^{A_{\beta} t} y_{0}\right\|_{Y_{\beta}} \leq M_{\beta} e^{-\omega_{\beta} t}\left\|y_{0}\right\|_{Y_{\beta}}$.

Step 1. As usual, we begin with a preliminary energy identity which shows that the system is dissipative.

Proposition 5.1. Assume Hypotheses 1.1, 1.2, and 1.3. With respect to the system (5.1), (5.2), and (5.3), the following energy equality holds true for all $T>0$ :

$$
\begin{aligned}
E_{\beta}(T) & +2 \int_{0}^{T}\left\|D_{0}^{1 / 2} N_{0}^{*} A_{N} z_{t}(t)\right\|^{2} d t \\
& +2 d_{2} \int_{0}^{T}\left\|z_{t}(t)\right\|^{2} d t+2 \rho \int_{0}^{T}\left\|\mathscr{A}^{\alpha / 2} v_{t}(t)\right\|^{2} d t=E_{\beta}(0) .
\end{aligned}
$$

In particular, $E_{\beta}(T) \leq E_{\beta}(0)$ for all $T>0$.

Proof. Multiplying the wave equation (5.2a) by $z_{t}$, we get, see (3.7),

$$
\begin{aligned}
\frac{1}{2} \frac{d}{d t}\left\{\left\|z_{t}\right\|^{2}\right. & \left.+\left\|A_{N}^{1 / 2} z\right\|^{2}+\beta\left\|D_{0}^{1 / 2} N_{0}^{*} A_{N} z\right\|^{2}\right\} \\
& +\left\|D_{0}^{1 / 2} N_{0}^{*} A_{N} z_{t}\right\|^{2}+d_{2}\left\|z_{t}\right\|^{2}-\left(v_{t}, N_{0}^{*} A_{N} z_{t}\right)=0 .
\end{aligned}
$$

After integration between 0 and $T$, then (5.6) yields the following identity for the wave energy $E_{z, \beta}$ in (5.3): for all $T>0$ we have

$$
\begin{aligned}
E_{z, \beta}(T) & +2 \int_{0}^{T}\left\|D_{0}^{1 / 2} N_{0}^{*} A_{N} z_{t}(t)\right\|^{2} d t \\
& +2 d_{2} \int_{0}^{T}\left\|z_{t}(t)\right\|^{2} d t-2 \int_{0}^{T}\left(v_{t}(t), N_{0}^{*} A_{N} z_{t}(t)\right) d t=E_{z, \beta}(0) .
\end{aligned}
$$

Analogously, multiplying the plate equation (5.2b) by $v_{t}$, we obtain (see (3.13) for $r=0$ )

$$
\frac{1}{2} \frac{d}{d t}\left\{\left\|v_{t}\right\|^{2}+\left\|\mathscr{A}^{1 / 2} v\right\|^{2}\right\}+\rho\left\|\mathscr{A}^{\alpha / 2} v_{t}\right\|^{2}+\left(N_{0}^{*} A_{N} z_{t}, v_{t}\right)=0
$$


Next, integrating (5.8) between 0 and $T$ yields the following identity for the plate energy $E_{v}$ in (5.3): for all $T>0$, we have

$$
E_{v}(T)+2 \rho \int_{0}^{T}\left\|\mathscr{A}^{\alpha / 2} v_{t}(t)\right\|^{2} d t+2 \int_{0}^{T}\left(N_{0}^{*} A_{N} z_{t}(t), v_{t}(t)\right) d t=E_{v}(0)
$$

Summing up (5.7) and (5.9) yields the energy equality (5.5) after a cancellation of the integral term.

In the following corollary, we point out a list of estimates which follow from Proposition 5.1, and which will play a crucial role later.

Corollary 5.2. Assume Hypothesis 1.1 with $d_{2}>0$, Hypotheses 1.2 and 1.3. For the components $z_{t}, v_{t}$ of the solution of system (5.1), the following estimates hold true: for any $T>0$, there exist constants $C_{1}, C_{2}$ which do not depend on $T$, such that

$$
\begin{aligned}
& \int_{0}^{T} E_{z, \beta}^{k}(t) d t \equiv \int_{0}^{T}\left\|z_{t}(t)\right\|_{L_{2}(\Omega)}^{2} d t \leq \frac{1}{2 d_{2}} E_{\beta}(0) ; \\
& \int_{0}^{T} E_{v}^{k}(t) d t \equiv \int_{0}^{T}\left\|v_{t}(t)\right\|_{L_{2}\left(\Gamma_{0}\right)}^{2} d t \\
& \leq\left\|\mathscr{A}^{-\alpha / 2}\right\|^{2} \int_{0}^{T}\left\|\mathscr{A}^{\alpha / 2} v_{t}(t)\right\|_{L_{2}\left(\Gamma_{0}\right)}^{2} d t \leq C_{1} E_{\beta}(0) ; \\
& E_{\beta}(T)+\int_{0}^{T}\left\|N_{0}^{*} A_{N} z_{t}(t)\right\|_{L_{2}\left(\Gamma_{0}\right)}^{2} d t \\
& \leq E_{\beta}(T)+\frac{\left\|\mathscr{A}^{-r_{0}}\right\|^{2}}{\delta_{1}} \int_{0}^{T}\left\|D_{0}^{1 / 2} N_{0}^{*} A_{N} z_{t}(t)\right\|_{L_{2}\left(\Gamma_{0}\right)}^{2} d t \\
& \leq C_{2} E_{\beta}(0) \text {. }
\end{aligned}
$$

Proof. Both estimates (5.10) and (5.11) on the kinetic energies $E_{z, \beta}^{k}$ and $K_{v}^{k}$ (defined above) of the wave and plate components readily follow from the energy identity (5.5); moreover, the first one (5.10) does require $d_{2}$ to be strictly positive. Finally, the latter estimate (5.12) is obtained first by using the left-hand side part of the estimates in (1.4) (from Hypothesis 1.3) on the damping operator $D_{0}$ to obtain (5.12a), and then by using (5.5) to reach (5.12b).

We now proceed with the estimates of the energy functional on a finite interval; first the plate energy in Proposition 5.3 and next the wave energy in Proposition 5.4.

Proposition 5.3. Assume Hypotheses 1.1, 1.2, and 1.3. With respect to the plate energy $E_{v}$ defined in (5.3), the following inequality holds true: for all $T>0$, there 
exist constants $C_{3}$ and $C_{4}$ independent of $T$, such that

$$
\int_{0}^{T} E_{v}(t) d t \leq C_{3}\left[E_{\beta}(0)+E_{v}(T)\right]+C_{4} \int_{0}^{T}\left\|N_{0}^{*} A_{N} z_{t}(t)\right\|_{L_{2}\left(\Gamma_{0}\right)}^{2} d t .
$$

Proof. We multiply the plate equation (5.2b) by the multiplier $v$ this time, then integrate between 0 and $T$, thus obtaining the difference "potential minus kinetic":

$$
\begin{aligned}
\left(v_{t}(T),\right. & v(T))-\int_{0}^{T}\left\|v_{t}(t)\right\|^{2} d t+\int_{0}^{T}\left\|\mathscr{A}^{1 / 2} v(t)\right\|^{2} d t+\frac{\rho}{2}\left\|\mathscr{A}^{\alpha / 2} v(t)\right\|^{2} \\
& +\int_{0}^{T}\left(\mathscr{A}^{-1 / 2} N_{0}^{*} A_{N} z_{t}(t), \mathscr{A}^{1 / 2} v(t)\right) d t=\left(v_{1}, v_{0}\right)+\frac{\rho}{2}\left\|\mathscr{A}^{\alpha / 2} v_{0}\right\|^{2}
\end{aligned}
$$

where the relevant norms are self-explanatory. Dropping the fourth (positive) term on the left-hand side of (5.14) and using the Schwarz inequality, we obtain (as $\alpha \leq 1$ ) recalling (5.3):

$$
\begin{aligned}
(1-\epsilon) & \left\{\int_{0}^{T}\left\|\mathscr{A}^{1 / 2} v(t)\right\|^{2} d t+\int_{0}^{T}\left\|v_{t}(t)\right\|^{2} d t\right\} \\
& \leq(2-\epsilon) \int_{0}^{T}\left\|v_{t}(t)\right\|^{2} d t+c_{1} E_{v}(T)+c_{2} E_{v}(0)+C_{\epsilon} \int_{0}^{T}\left\|N_{0}^{*} A_{N} z_{t}(t)\right\|^{2} d t .
\end{aligned}
$$

Finally, on the left-hand side of (5.15) we use (5.3), for $E_{v}(t)$ and on the righthand side of (5.15) we invoke estimate (5.11) for its first integral term as well as $E_{v}(0) \leq E_{\beta}(0)$, to obtain (5.13), as desired.

Proposition 5.4. Assume Hypothesis 1.1, this time with $d_{2}>0$, Hypotheses 1.2 and 1.3.

Case 1. Assume further that

$$
D_{0}^{1 / 2} \text { : continuous } H^{1 / 2}\left(\Gamma_{0}\right) \longrightarrow L_{2}\left(\Gamma_{0}\right),
$$

and let $\beta=0$ in (5.1c). Then, the energy $E_{z, \beta=0}(t)=\left\|A_{N}^{1 / 2} z(t)\right\|^{2}+\left\|z_{t}(t)\right\|^{2}$ defined in (5.3) satisfies the following estimate for some $c_{i}>0$ :

$$
\int_{0}^{T} E_{z, \beta=0}(t) d t \leq\left[\frac{c_{1}}{d_{2}}+c_{2}\right] E_{\beta=0}(0)+c_{3} E_{z, \beta=0}(T),
$$

with $E_{\beta=0}(t)$ defined in (5.3).

Case 2. Assume now that $D_{0}$ is of order $s, 1<s \leq 2$. Take now $\beta>0$. Then the corresponding energy $E_{z, \beta}(t)$ in (5.3) satisfies the following estimate for $c_{i}>0$ :

$$
\int_{0}^{T} E_{z, \beta}(t) d t \leq\left[\frac{c_{4}}{d_{2}}+\frac{c_{5}}{\beta}+c_{6}\right] E_{\beta}(0)+\frac{c_{7}}{\beta} E_{z, \beta}(T),
$$

with $E_{\beta}(t)$ defined in (5.3). 
218 Singular estimates and uniform stability

Proof. We multiply the wave equation (5.2a) by the multiplier $z$ this time, then integrate between 0 and $T$, thus obtaining the difference "potential minus kinetic":

$$
\begin{aligned}
& \left(z_{t}(T), z(T)\right)-\int_{0}^{T}\left\|z_{t}(t)\right\|^{2} d t \\
& \quad+\int_{0}^{T}\left\|A_{N}^{1 / 2} z(t)\right\|^{2} d t+\beta \int_{0}^{T}\left\|D_{0}^{1 / 2} N_{0}^{*} A_{N} z(t)\right\|^{2} d t \\
& \quad+\frac{1}{2}\left\{\left\|D_{0}^{1 / 2} N_{0}^{*} A_{N} z(T)\right\|^{2}+d_{2}\|z(T)\|^{2}\right\}-\int_{0}^{T}\left(v_{t}(t), N_{0}^{*} A_{N} z(t)\right) d t \\
& =\left(z_{1}, z_{0}\right)+\frac{1}{2}\left\{\left\|D_{0}^{1 / 2} N_{0}^{*} A_{N} z_{0}\right\|^{2}+d_{2}\left\|z_{0}\right\|^{2}\right\} .
\end{aligned}
$$

We now distinguish between the two cases assumed.

Case 1. Assume (5.16) and take $\beta=0$. By (5.16), it follows that

$$
D_{0}^{1 / 2} N_{0}^{*} A_{N}^{1 / 2}=D_{0}^{1 / 2}\left(N_{0}^{*} A_{N}\right) A_{N}^{-1 / 2}: \text { continuous } L_{2}(\Omega) \longrightarrow L_{2}\left(\Gamma_{0}\right)
$$

Indeed, with $f \in L_{2}(\Omega)$, then $A_{N}^{-1 / 2} f \in \mathscr{D}\left(A_{N}^{1 / 2}\right) \equiv H^{1}(\Omega)$, and hence $\left(N_{0}^{*} A_{N}\right) \times$ $A_{N}^{-1 / 2} f \in H^{1 / 2}\left(\Gamma_{0}\right)$ continuously, by (2.6) and trace theory, and (5.16) then yields (5.20), as desired. It follows from (5.20) and $N_{1}^{*} A_{N}^{1 / 2} \in \mathscr{L}\left(L_{2}(\Omega) ; L_{2}\left(\Gamma_{1}\right)\right)$ by (2.6), that the terms $\{\quad\}$ in (5.19) satisfy the estimate

$$
\begin{aligned}
\left\{\left\|\left(D_{0}^{1 / 2} N_{0}^{*} A_{N}^{1 / 2}\right) A_{N}^{1 / 2} z(t)\right\|^{2}+d_{2} \|\left(N_{1}^{*} A_{N}^{1 / 2}\right)\right. & \left.A_{N}^{1 / 2} z(t)\left\|^{2}+d_{2}\right\| z(t) \|^{2}\right\} \\
& \leq C\left\|A_{N}^{1 / 2} z(t)\right\|^{2} \leq c E_{z, \beta=0}(t) .
\end{aligned}
$$

Using (5.21) for $t=0$ and $t=T$ and given $\epsilon>0$, we then obtain the following estimate from (5.19) with $\beta=0$ :

$$
\begin{aligned}
\int_{0}^{T}\left\|A_{N}^{1 / 2} z(t)\right\|^{2} d t \leq & \int_{0}^{T}\left\|z_{t}(t)\right\|^{2} d t+c\left[E_{z, \beta=0}(T)+E_{z, \beta=0}(0)\right] \\
& +\epsilon\left\|N_{0}^{*} A^{1 / 2}\right\| \int_{0}^{T}\left\|A_{N}^{1 / 2} z(t)\right\|^{2} d t+c_{\epsilon} \int_{0}^{T}\left\|v_{t}(t)\right\|^{2} d t .
\end{aligned}
$$


With $\epsilon^{\prime}=\epsilon\left\|N_{0}^{*} A_{N}^{1 / 2}\right\|$ small, (5.22) then yields

$$
\begin{aligned}
& \left(1-\epsilon^{\prime}\right)\left\{\int_{0}^{T}\left\|A_{N}^{1 / 2} z(t)\right\|^{2} d t+\int_{0}^{T}\left\|z_{t}(t)\right\|^{2} d t\right\} \\
& \quad \leq\left(2-\epsilon^{\prime}\right) \int_{0}^{T}\left\|z_{t}(t)\right\|^{2} d t+c\left[E_{z, \beta=0}(T)+E_{z, \beta=0}(0)\right]+C_{\epsilon} \int_{0}^{T}\left\|v_{t}(t)\right\|^{2} d t
\end{aligned}
$$

(by (5.10) and (5.11))

$$
\leq\left[\frac{2-\epsilon^{\prime}}{2 d_{2}}+C_{\epsilon} C_{1}\right] E_{\beta=0}(0)+c\left[E_{z, \beta=0}(T)+E_{z, \beta=0}(0)\right] .
$$

To go from (5.23) to (5.24) we have invoked estimates (5.10) and (5.11) for the integral terms on $z_{t}$ and $v_{t}$. (In (5.24), we have deliberately put into evidence the dependence of the estimate on $d_{2}>0$.) Finally, (5.24) yields (5.17) as desired, by recalling $E_{z, \beta=0}(t)$ in (5.3) on the left-hand side of (5.24) and $E_{z, \beta=0}(0) \leq E_{\beta=0}(0)$ on its right-hand side.

Case 2. Returning to the terms \{\} on the right-hand side of (5.19), we now have since $\beta>0$,

$$
\begin{aligned}
\left\{\frac{1}{\beta} \beta\right. & \left.\left\|D_{0}^{1 / 2} N_{0}^{*} A_{N} z(t)\right\|^{2}+d_{2}\|z(t)\|^{2}\right\} \\
& \leq \frac{c}{\beta}\left[\left\|A_{N}^{1 / 2} z(t)\right\|^{2}+\beta\left\|D_{0}^{1 / 2} N_{0}^{*} A_{N} z(t)\right\|^{2}\right] \leq \frac{c}{\beta} E_{z, \beta}(t)
\end{aligned}
$$

in contrast with (5.21). Thus, from (5.19) and (5.26), the counterpart of (5.22) is now

$$
\begin{aligned}
& \int_{0}^{T}\left\|A_{N}^{1 / 2} z(t)\right\|^{2} d t+\int_{0}^{T} \beta\left\|D_{0}^{1 / 2} N_{0}^{*} A_{Z} z(t)\right\|^{2} d t \\
& \leq \int_{0}^{T}\left\|z_{t}(t)\right\|^{2} d t+\frac{c}{\beta}\left[E_{z, \beta}(T)+E_{z, \beta}(0)\right] \\
& \quad+\epsilon\left\|N_{0}^{*} A_{N}^{1 / 2}\right\| \int_{0}^{T}\left\|A_{N}^{1 / 2} z(t)\right\|^{2} d t+C_{\epsilon} \int_{0}^{T}\left\|v_{t}(t)\right\|^{2} d t
\end{aligned}
$$

while the counterpart of (5.23) is now, still with $\epsilon^{\prime}=\epsilon\left\|N_{0}^{*} A_{N}^{1 / 2}\right\|$ small:

$$
\begin{aligned}
& \left(1-\epsilon^{\prime}\right) \int_{0}^{T}\left[\left\|A_{N}^{1 / 2} z(t)\right\|^{2}+\beta\left\|D_{0}^{1 / 2} N_{0}^{*} A_{N} z(t)\right\|^{2}+\left\|z_{t}(t)\right\|^{2}\right] d t \\
& \quad \leq\left(2-\epsilon^{\prime}\right) \int_{0}^{T}\left\|z_{t}(t)\right\|^{2} d t+\frac{c}{\beta}\left[E_{z, \beta}(T)+E_{z, \beta}(0)\right]+C_{\epsilon} \int_{0}^{T}\left\|v_{t}(t)\right\|^{2} d t
\end{aligned}
$$

(by (5.10) and (5.11))

$$
\leq\left[\frac{2-\epsilon^{\prime}}{2 d_{2}}+C_{\epsilon} c_{1}\right] E_{\beta}(0)+\frac{c}{\beta}\left[E_{z, \beta}(T)+E_{z, \beta}(0)\right]
$$


Again, to go from (5.27) to (5.28) we have invoked (5.10), (5.11) on the integral terms on $z_{t}$ and $v_{t}$. (Again, in (5.29), (5.30) we have deliberately put into evidence the dependence of the estimates on $d_{2}>0$ and $\beta>0$.) Finally, (5.28) yields (5.18), as desired, by recalling $E_{\beta}(t)$ in (5.3) on the left-hand side of (5.28), and $E_{z, \beta}(0) \leq E_{\beta}(0)$ on its right-hand side.

Proposition 5.5. Assume the situation of Proposition 5.3. Then, there are two cases.

Case $1\left(\beta=0 ; d_{2}>0\right)$

$$
\int_{0}^{T} E_{\beta=0}(t) d t \leq\left[\frac{c_{1}^{\prime}}{d_{2}}+c_{2}^{\prime}\right] E_{\beta=0}(0) .
$$

Case $2\left(\beta>0 ; d_{2}>0\right)$

$$
\int_{0}^{T} E_{\beta}(t) d t \leq\left[\frac{c_{4}^{\prime}}{d_{2}}+\frac{c_{5}^{\prime}}{\beta}+c_{6}^{\prime}\right] E_{\beta}(0) .
$$

Proof

Case 1. We sum up estimates (5.13) and (5.17) to get via (5.3),

$$
\begin{aligned}
\int_{0}^{T} E_{\beta=0}(t) d t \leq & {\left[\frac{c_{1}^{\prime}}{d_{2}}+c_{2}^{\prime}\right] E_{\beta=0}(0) } \\
& +\left\{C_{3} E_{v}(T)+c_{3} E_{z, \beta=0}(T)+c_{4} \int_{0}^{T}\left\|N_{0}^{*} A_{N} z_{t}(t)\right\|_{L_{2}\left(\Gamma_{0}\right)}^{2} d t\right\} \\
(\operatorname{by}(5.12)) \leq & {\left[\frac{c_{1}^{\prime}}{d_{2}}+c_{2}^{\prime \prime}\right] E_{\beta=0}(0), }
\end{aligned}
$$

where in the last step we have invoked estimate (5.12) for the \{\} -term in (5.31). Then, (5.32) proves (5.29), as desired.

Case 2. We sum up estimates (5.13) and (5.18) to obtain via (5.3)

$$
\begin{aligned}
\int_{0}^{T} E_{\beta}(t) d t \leq & {\left[\frac{c_{4}^{\prime}}{d_{2}}+\frac{c_{5}^{\prime}}{\beta}+c_{6}^{\prime}\right] E_{\beta}(0) } \\
& +\left\{C_{3} E_{v}(T)+\frac{c_{7}}{\beta} E_{z, \beta}(T)+\int_{0}^{T}\left\|N_{0}^{*} A_{N} z_{t}(t)\right\|_{L_{2}\left(\Gamma_{0}\right)}^{2} d t\right\} \\
(\text { by }(5.12)) \leq & {\left[\frac{c_{4}^{\prime}}{d_{2}}+\frac{c_{5}^{\prime \prime}}{\beta}+c_{6}^{\prime}\right] E_{\beta}(0), }
\end{aligned}
$$

where in the last step we have invoked estimate (5.12) for the \{\} -term in (5.33). Then, (5.34) proves (5.30), as desired.

Finally, Proposition 5.4 proves the desired goal (5.4) in both cases. Hence, by Datko's theorem [32, page 116] there exists constants $M_{\beta} \geq 1, \omega_{\beta}>0$ such that 
(1.21) holds true:

$$
\left\|e^{A_{\beta} t}\right\|_{\mathscr{L}\left(Y_{\beta}\right)} \leq M_{\beta} e^{-\omega_{\beta} t}, \quad t \geq 0 .
$$

6. Theorem 1.14: uniform stability of problem (1.2) with $f \equiv 0, u \equiv 0$ via the resolvent condition

We return to problem (1.2), hence to its abstract version (2.40) with $f \equiv 0, u \equiv 0$, identified by the operator $A$ in (2.37) and (2.38). To emphasize its dependence on $\beta$, we will now append a subscript and write $A_{\beta}=A=$ given by (2.37) and (2.38) as in Section 1.4. Recall

$$
\begin{aligned}
& Y_{\beta} \equiv Z_{\beta} \times L_{2}(\Omega) \times \mathscr{D}\left(\mathscr{A}^{1 / 2}\right) \times L_{2}\left(\Gamma_{0}\right), \quad \beta \geq 0 \text {, with norm given by } \\
& \left\{\left\|A_{N}^{1 / 2} z_{1}\right\|^{2}+\beta\left\|D_{0}^{1 / 2} N_{0}^{*} A_{N} z_{1}\right\|^{2}+\left\|\mathscr{A}^{1 / 2} v_{1}\right\|^{2}+\left\|v_{2}\right\|^{2}\right\}^{1 / 2} \equiv\left\|\left\{z_{1}, z_{2}, v_{1}, v_{2}\right\}\right\|_{Y_{\beta}} .
\end{aligned}
$$

The goal of Section 6 is to show Theorem 1.14, which is here restated for convenience.

Theorem 6.1. Consider problem (1.2) with $f \equiv 0, u \equiv 0$ (i.e., problem (5.1)) on the space $Y_{\beta}$ under assumptions that $d_{1} \geq 0, d_{2}>0,0 \leq \alpha \leq 1, \rho>0$, and (1.4) and (1.5). The case $\alpha=0, D_{0}=0$ is explicitly included. Let $\beta \geq 0$.

(i) Given $\epsilon>0$, there exists $C_{\epsilon}>0$ such that, for all $\omega \in \mathbb{R}$ with $|\omega| \geq \epsilon$, then the operator $A=A_{\beta}$ defined by (2.37) and (2.38) satisfies the following resolvent condition on the imaginary axis:

$$
\left\|R\left(i \omega, A_{\beta}\right)\right\|_{\mathscr{L}\left(Y_{\beta}\right)} \leq C_{\epsilon, \beta}, \quad \forall \omega \in \mathbb{R} \text { s.t. }|\omega| \geq \epsilon .
$$

(ii) Assume that $\lambda=0$ is in the resolvent set of $A_{\beta}$. By Proposition 4.3 this holds true in the two cases mentioned in Theorem 1.13 or Theorem 1.14 under Assumptions 1.1 and 1.3:

Case 1. $D_{0}$ is of order up to 1 and $\beta=0$.

Case 2. $D_{0}$ is of order $s, 1<s \leq 2$ and $\beta>0$.

Then estimate (6.2) holds true with $\epsilon=0$, that is, for all $\omega \in \mathbb{R}$. Accordingly $([9,30,33])$, there exist constants $M_{\beta} \geq 1$ and $k_{\beta}>0$ such that

$$
\left\|e^{A_{\beta} t}\right\|_{\mathscr{L}\left(Y_{\beta}\right)} \leq M_{\beta} e^{-k_{\beta} t}, \quad t \geq 0
$$

(see Theorem 1.6). The case $\alpha=0, D_{0}=0$ is also included.

Proof. (i) We will write the proof simultaneously for all $\beta \geq 0,0 \leq \alpha \leq 1$, and $D_{0}$ as in Hypothesis 1.3, possibly $D_{0}=0$. 
Step 1. Given $f=\left[f_{1}, f_{2}, f_{3}, f_{4}\right] \in Y_{\beta}$ in (6.1), we seek to solve

$$
\left(i \omega I-A_{\beta}\right)\left[\begin{array}{l}
z_{1} \\
z_{2} \\
v_{1} \\
v_{2}
\end{array}\right]=\left[\begin{array}{l}
f_{1} \\
f_{2} \\
f_{3} \\
f_{4}
\end{array}\right], \text { to obtain }\left[\begin{array}{l}
z_{1} \\
z_{2} \\
v_{1} \\
v_{2}
\end{array}\right]=R\left(i \omega, A_{\beta}\right)\left[\begin{array}{l}
f_{1} \\
f_{2} \\
f_{3} \\
f_{4}
\end{array}\right]
$$

for $\left[z_{1}, z_{2}, v_{1}, v_{2}\right] \in \mathscr{D}\left(A_{\beta}\right)$, where, in addition, we seek to obtain the following estimate for $\left[z_{1}(\omega), z_{2}(\omega), v_{1}(\omega), v_{2}(\omega)\right]$ : given $\epsilon>0$, there exists $C_{\epsilon}>0$ such that for all $\omega \in \mathbb{R}$ with $|\omega| \geq \epsilon$, we have

$$
\begin{gathered}
\left\|A_{N}^{1 / 2} z_{1}(\omega)\right\|_{L_{2}(\Omega)}^{2}+\beta\left\|D_{0}^{1 / 2} N_{0}^{*} A_{N} z_{1}(\omega)\right\|_{L_{2}\left(\Gamma_{0}\right)}^{2}+\left\|z_{2}(\omega)\right\|_{L_{2}(\Omega)}^{2} \\
+\left\|\mathscr{A}^{1 / 2} v_{1}(\omega)\right\|_{L_{2}\left(\Gamma_{0}\right)}^{2}+\left\|v_{2}(\omega)\right\|_{L_{2}\left(\Gamma_{0}\right)}^{2} \leq C_{\epsilon}\|f\|_{Y_{\beta}}^{2}, \\
\|f\|_{Y_{\beta}}^{2}=\{ \\
+\left\|A_{N}^{1 / 2} f_{1}\right\|_{L_{2}(\Omega)}^{2}+\beta\left\|D_{0}^{1 / 2} N_{0}^{*} A_{N} f_{1}\right\|_{L_{2}\left(\Gamma_{0}\right)}^{2} \\
\left.+\left\|f_{2}\right\|_{L_{2}(\Omega)}^{2}+\left\|\mathscr{A}^{1 / 2} f_{3}\right\|_{L_{2}\left(\Gamma_{0}\right)}^{2}+\left\|f_{4}\right\|_{L_{2}\left(\Gamma_{0}\right)}^{2}\right\} .
\end{gathered}
$$

Recalling (2.37) and (2.38) for $A=A_{\beta}$, we rewrite (6.5) explicitly as

$$
\begin{gathered}
i \omega z_{1}-z_{2}=f_{1} ; \\
A_{N}\left[z_{1}+\beta N_{0} D_{0} N_{0}^{*} A_{N} z_{1}+N_{0} D_{0} N_{0}^{*} A_{N} z_{2}\right]+\left(i \omega+d_{2}\right) z_{2}-A_{N} N_{0} v_{2}=f_{2} ; \\
i \omega v_{1}-v_{2}=f_{3} ; \\
N_{0}^{*} A_{N} z_{2}+\mathscr{A} v_{1}+\rho \mathcal{A}^{\alpha} v_{2}+i \omega v_{2}=f_{4} .
\end{gathered}
$$

Taking the $L_{2}(\Omega)$-inner product of (6.7b) with $z_{2}$, and, respectively, the $L_{2}\left(\Gamma_{0}\right)$ inner product of $(6.7 \mathrm{~d})$ with $v_{2}$ yields

$$
\begin{aligned}
\left(A_{N}^{1 / 2} z_{1}, A_{N}^{1 / 2} z_{2}\right)+\beta( & \left.D_{0} N_{0}^{*} A_{N} z_{1}, N_{0}^{*} A_{N} z_{2}\right)+\left\|D_{0}^{1 / 2} N_{0}^{*} A_{N} z_{2}\right\|^{2} \\
& +i \omega\left\|z_{2}\right\|^{2}+d_{2}\left\|z_{2}\right\|^{2}-\left(A_{N} N_{0} v_{2}, z_{2}\right)=\left(f_{2}, z_{2}\right)
\end{aligned}
$$

where inner products and norms are in $L_{2}(\Omega)$ or $L_{2}\left(\Gamma_{0}\right)$.

Step 2. We begin by substituting $z_{2}=i \omega z_{1}-f_{1}$ from (6.7a) into the first two terms of (6.8), thus obtaining

$$
\begin{aligned}
-i \omega \| & A_{N}^{1 / 2} z_{1}\left\|^{2}-i \omega \beta\right\| D_{0}^{1 / 2} N_{0}^{*} A_{N} z_{1}\left\|^{2}+\right\| D_{0}^{1 / 2} N_{0}^{*} A_{N} z_{2} \|^{2} \\
& +i \omega\left\|z_{2}\right\|^{2}+d_{2}\left\|z_{2}\right\|^{2}-\left(v_{2}, N_{0}^{*} A_{N} z_{2}\right) \\
= & \left(f_{2}, z_{2}\right)+\left(A_{N}^{1 / 2} z_{1}, A_{N}^{1 / 2} f_{1}\right)+\beta\left(D_{0}^{1 / 2} N_{0}^{*} A_{N} z_{1}, D_{0}^{1 / 2} N_{0}^{*} A_{N} f_{1}\right) .
\end{aligned}
$$


Similarly, we substitute $v_{2}=i \omega v_{1}-f_{3}$ from (6.7c) into the second term of (6.9), thus obtaining

$$
\begin{gathered}
\left(N_{0}^{*} A_{N} z_{2}, v_{2}\right)-i \omega\left\|\mathscr{A}^{1 / 2} v_{1}\right\|^{2}+\rho\left\|\mathscr{A}^{\alpha / 2} v_{2}\right\|^{2}+i \omega\left\|v_{2}\right\|^{2} \\
=\left(f_{4}, v_{2}\right)+\left(\mathscr{A}^{1 / 2} v_{1}, \mathscr{A}^{1 / 2} f_{3}\right) .
\end{gathered}
$$

Next, we take the real part of (6.10) and (6.11), thus obtaining

$$
\begin{aligned}
& \left\|D_{0}^{1 / 2} N_{0}^{*} A_{N} z_{2}\right\|^{2}+d_{2}\left\|z_{2}\right\|^{2}-\operatorname{Re}\left(v_{2}, N_{0}^{*} A_{N} z_{2}\right) \\
& \quad=\operatorname{Re}\left\{\left(f_{2}, z_{2}\right)+\left(A_{N}^{1 / 2} z_{1}, A_{N}^{1 / 2} f_{1}\right)+\beta\left(D_{0}^{1 / 2} N_{0}^{*} A_{N} z_{1}, D_{0}^{1 / 2} N_{0}^{*} A_{N} f_{1}\right)\right\} \\
& \operatorname{Re}\left(N_{0}^{*} A_{N} z_{2}, v_{2}\right)+\rho\left\|\mathscr{A}^{\alpha / 2} v_{2}\right\|^{2}=\operatorname{Re}\left\{\left(f_{4}, v_{2}\right)+\left(\mathscr{A}^{1 / 2} v_{1}, \mathscr{A}^{1 / 2} f_{3}\right)\right\} .
\end{aligned}
$$

Similarly, we take the imaginary part of (6.10) and (6.11), thus obtaining

$$
\begin{gathered}
\omega\left[\left\|z_{2}\right\|^{2}-\left\|A_{N}^{1 / 2} z_{1}\right\|^{2}-\beta\left\|D_{0}^{1 / 2} N_{0}^{*} A_{N} z_{1}\right\|^{2}\right]-\operatorname{Im}\left(v_{2}, N_{0}^{*} A_{N} z_{2}\right) \\
\quad=\operatorname{Im}\left\{\left(f_{2}, z_{2}\right)+\left(A_{N}^{1 / 2} z_{1}, A_{N}^{1 / 2} f_{1}\right)+\beta\left(D_{0}^{1 / 2} N_{0}^{*} A_{N} z_{1}, D_{0}^{1 / 2} N_{0}^{*} A_{N} f_{1}\right)\right\} \\
\operatorname{Im}\left(N_{0}^{*} A_{N} z_{2}, v_{2}\right)+\omega\left[\left\|v_{2}\right\|^{2}-\left\|\mathscr{A}^{1 / 2} v_{1}\right\|^{2}\right]=\operatorname{Im}\left\{\left(f_{4}, v_{2}\right)+\left(\mathscr{A}^{1 / 2} v_{1}, \mathscr{A}^{1 / 2} f_{3}\right)\right\}
\end{gathered}
$$

Next, we sum up (6.12) and (6.13), thus cancelling $\operatorname{Re}\left(N_{0}^{*} A_{N} z_{2}, v_{2}\right)=\operatorname{Re}\left(v_{2}\right.$, $\left.N_{0}^{*} A_{N} z_{2}\right)$; and, similarly, we sum up (6.14) and (6.15), where

$$
\operatorname{Im}\left(N_{0}^{*} A_{N} z_{2}, v_{2}\right)=-\operatorname{Im}\left(v_{2}, N_{0}^{*} A_{N} z_{2}\right) .
$$

We obtain, respectively,

$$
\begin{gathered}
\left\|D_{0}^{1 / 2} N_{0}^{*} A_{N} z_{2}\right\|^{2}+d_{2}\left\|z_{2}\right\|^{2}+\rho\left\|\mathscr{A}^{\alpha / 2} v_{2}\right\|^{2} \\
=\operatorname{Re}\left\{\left(f_{2}, z_{2}\right)+\left(A_{N}^{1 / 2} z_{1}, A_{N}^{1 / 2} f_{1}\right)\right. \\
+\beta\left(D_{0}^{1 / 2} N_{0}^{*} A_{N} z_{1}, D_{0}^{1 / 2} N_{0}^{*} A_{N} f_{1}\right) \\
\left.+\left(\mathscr{A}^{-\alpha / 2} f_{4}, \mathscr{A}^{\alpha / 2} v_{2}\right)+\left(\mathscr{A}^{1 / 2} v_{1}, \mathscr{A}^{1 / 2} f_{3}\right)\right\} ; \\
\omega\left[\left\|A_{N}^{1 / 2} z_{1}\right\|^{2}+\beta\left\|D_{0}^{1 / 2} N_{0}^{*} A_{N} z_{1}\right\|^{2}+\left\|\mathscr{A}^{1 / 2} v_{1}\right\|^{2}\right] \\
=\omega\left[\left\|z_{2}\right\|^{2}+\left\|v_{2}\right\|^{2}\right]+2 \operatorname{Im}\left(N_{0}^{*} A_{N} z_{2}, v_{2}\right) \\
-\operatorname{Im}\left\{\left(f_{2}, z_{2}\right)+\left(A_{N}^{1 / 2} z_{1}, A_{N}^{1 / 2} f_{1}\right)\right. \\
+\beta\left(D_{0}^{1 / 2} N_{0}^{*} A_{N} z_{1}, D_{0}^{1 / 2} N_{0}^{*} A_{N} f_{1}\right) \\
\left.+\left(f_{4}, v_{2}\right)+\left(\mathscr{A}^{1 / 2} v_{1}, \mathscr{A}^{1 / 2} f_{3}\right)\right\} .
\end{gathered}
$$


From (6.17), we estimate, thus obtaining

$$
\begin{aligned}
\left\|D_{0}^{1 / 2} N_{0}^{*} A_{N} z_{2}\right\|^{2}+\left(d_{2}-\frac{\epsilon}{2}\right)\left\|z_{2}\right\|^{2}+\left(\rho-\frac{\epsilon}{2}\right)\left\|\mathscr{A}^{\alpha / 2} v_{2}\right\|^{2} \\
\leq \frac{\epsilon}{2}\left[\left\|A_{N}^{1 / 2} z_{1}\right\|^{2}+\beta\left\|D_{0}^{1 / 2} N_{0}^{*} A_{N} z_{1}\right\|^{2}+\left\|\mathscr{A}^{1 / 2} v_{1}\right\|^{2}\right] \\
\quad+\frac{1}{2 \epsilon}\left[\left\|A_{N}^{1 / 2} f_{1}\right\|^{2}+\left\|f_{2}\right\|^{2}+\beta\left\|D_{0}^{1 / 2} N_{0}^{*} A_{N} f_{1}\right\|^{2}+\left\|\mathscr{A}^{-\alpha / 2} f_{4}\right\|^{2}+\left\|\mathscr{A}^{1 / 2} f_{3}\right\|^{2}\right] .
\end{aligned}
$$

Recalling $z_{2}=i \omega z_{1}-f_{1}$ from $(6.7 \mathrm{a})$, and setting $C=\left\|N_{0}^{*} A_{N}^{1 / 2}\right\|($ see $(2.4))$, we estimate

$$
\begin{aligned}
2\left|\operatorname{Im}\left(N_{0}^{*} A_{N} z_{2}, v_{2}\right)\right| & \leq 2|\omega|^{1 / 2}\left\|A_{N}^{1 / 2} z_{1}\right\| C|\omega|^{1 / 2}\left\|v_{2}\right\|+2 C\left\|A_{N}^{1 / 2} f_{1}\right\|\left\|v_{2}\right\| \\
& \leq \epsilon_{1}|\omega|\left\|A_{N}^{1 / 2} z_{1}\right\|^{2}+\frac{C^{2}|\omega|}{\epsilon_{1}}\left\|v_{2}\right\|^{2}+\frac{\epsilon}{2}\left\|v_{2}\right\|^{2}+\frac{2 C^{2}}{\epsilon}\left\|A_{N}^{1 / 2} f_{1}\right\|^{2}
\end{aligned}
$$

Next, we estimate (6.18), and use (6.20) on its right-hand side, thus obtaining

$$
\begin{aligned}
{\left[|\omega|\left(1-\epsilon_{1}\right)-\frac{\epsilon}{2}\right]\left[\left\|A_{N}^{1 / 2} z_{1}\right\|^{2}+\beta\left\|D_{0}^{1 / 2} N_{0}^{*} A_{N} z_{1}\right\|^{2}+\left\|\mathscr{A}^{1 / 2} v_{1}\right\|^{2}\right] } \\
\leq\left[|\omega|\left(1+\frac{C^{2}}{\epsilon_{1}}\right)+\epsilon\right]\left[\left\|z_{2}\right\|^{2}+\left\|v_{2}\right\|^{2}\right]+C_{\epsilon}\|f\|_{Y_{\beta}}^{2} .
\end{aligned}
$$

From (6.19), with $m_{\epsilon}=\min \left\{d_{2}-\epsilon / 2, k_{\alpha}(\rho-\epsilon / 2)\right\}>0, k_{\alpha}=1 /\left\|A^{-\alpha / 2}\right\|^{2}$, we obtain a fortiori, for all $0 \leq \alpha \leq 1$ :

$$
\left\|z_{2}\right\|^{2}+\left\|v_{2}\right\|^{2} \leq \frac{\epsilon}{2 m_{\epsilon}}\left[\left\|A_{N}^{1 / 2} z_{1}\right\|^{2}+\beta\left\|D_{0}^{1 / 2} N_{0}^{*} A_{N} z_{1}\right\|^{2}+\left\|\mathscr{A}^{1 / 2} v_{1}\right\|^{2}\right]+\frac{C_{\epsilon}}{m_{\epsilon}}\|f\|_{Y_{\beta}}^{2} .
$$

Using (6.22) on the right-hand side of (6.21) yields

$$
\left[|\omega|\left(1-\delta_{\epsilon}\right)-a_{\epsilon}\right]\left[\left\|A_{N}^{1 / 2} z_{1}\right\|^{2}+\beta\left\|D_{0}^{1 / 2} N_{0}^{*} A_{N} z_{1}\right\|^{2}+\left\|A^{1 / 2} v_{1}\right\|^{2}\right] \leq C_{\epsilon}\|f\|_{Y_{\beta}}^{2},
$$

where by taking, say $\epsilon_{1}=\sqrt{\epsilon}$ we have $\delta_{\epsilon}=\epsilon_{1}+\epsilon / 2 m_{\epsilon}+\epsilon C^{2} / 2 m_{\epsilon} \epsilon_{1}=\sqrt{\epsilon}+$ $\epsilon / 2 m_{\epsilon}+\left(\sqrt{\epsilon} / 2 m_{\epsilon}\right) C^{2} \rightarrow 0$, and $a_{\epsilon}=\epsilon / 2+\epsilon^{2} / 2 m_{\epsilon} \rightarrow 0$, as $\epsilon \searrow 0$. So $a_{\epsilon} /\left(1-\delta_{\epsilon}\right) \rightarrow$ 0 as $\epsilon \searrow 0$, as desired. Thus, (6.23) shows estimate (6.5) for the terms $\left\|A_{N}^{1 / 2} z_{1}\right\|^{2}$, $\beta\left\|D_{0}^{1 / 2} N_{0}^{*} A_{N} z_{1}\right\|^{2}$, and $\left\|\mathscr{A}^{1 / 2} v_{1}\right\|^{2}$. Finally, using (6.23) on the right-hand side of (6.19) show the desired estimate (6.5) also for the terms $\left\|z_{2}\right\|^{2}$ and $\left\|v_{2}\right\|^{2}$; and indeed, for $\left\|D_{0}^{1 / 2} N_{0}^{*} A_{N} z_{2}\right\|^{2}$ as well. The proof of part (i) is complete. The above proof is valid also for $\alpha=0$ and $D_{0}=0$.

(ii) This part follows at once via the quoted references, whenever $\lambda=0$ is in the resolvent set of $A_{\beta}$, and $A_{\beta}$ is a generator of a s.c. semigroup $e^{A_{\beta} t}$ on $Y_{\beta}$ (see Theorem 1.6, under Hypotheses 1.1 and 1.3); but this is true also for $D_{0}=0$ and $\alpha=0$. 


\section{Appendices}

\section{A. Proof of Theorem 1.6 (well-posedness)}

The goal of this Appendix A is to establish semigroup well-posedness of system (1.2), or (2.40), when $\tilde{u} \equiv 0, F \equiv 0$. As a preliminary step, we provide the proof of Lemma 2.4 on the semigroup well-posedness of the $z$-problem (1.2a), (1.2b), and (1.2c), equivalently of problem (2.7a) with $f \equiv 0$ and $v_{t}$ set equal to zero in (1.2c) or (2.7b). We have seen that it is the high damping term $D_{0} z_{t}$ in (1.2c), hence the term $A_{N} N_{0} D_{0} N_{0}^{*} A_{N}$ on the second coordinate that makes this problem non-standard. In particular, the present result is not contained in the generation theorem in [19, Proposition 7.6.2.1, Chapter 7, page 664].

Step 1 (Proof of Lemma 2.4). It is easily verified that $A_{1}$ and $A_{1}^{*}$ defined in (2.12) and (2.14), respectively, are densely defined and dissipative on $Y_{1}=Z_{\beta} \times L_{2}(\Omega)$ defined by (2.10). Dissipativity is critically dependent on the choice of the norm (inner product) which was made in (2.9) for $Z_{\beta}$ precisely to achieve dissipativity via a cancellation:

$$
\begin{array}{r}
\operatorname{Re}\left(A_{1} x, x\right)_{Y_{1}}=\operatorname{Re}\left(A_{1}^{*} x, x\right)_{Y_{1}}=-\left\|D_{0}^{1 / 2} N_{0}^{*} A_{N} x_{2}\right\|_{L_{2}\left(\Gamma_{0}\right)}^{2}-d_{2}\left\|x_{2}\right\|_{L_{2}(\Omega)}^{2} \leq 0, \\
x=\left[x_{1}, x_{2}\right] \in \mathscr{D}\left(A_{1}\right)=\mathscr{D}\left(A_{1}^{*}\right) .
\end{array}
$$

Thus, all we need to show is that $A_{1}$ is (not only closable but in fact) closed, and then invoke a corollary of Lumer-Phillips theorem (cf. [32, page 14]) and obtain Lemma 2.4. To show that $A_{1}$ is closed, let via (2.12b)

$$
\begin{gathered}
z^{n} \equiv\left[z_{1}^{n}, z_{2}^{n}\right] \in \mathscr{D}\left(A_{1}\right) \subset Z_{\beta} \times Z_{\beta} ; \\
z^{n} \longrightarrow z=\left[z_{1}, z_{2}\right] \text { in } Y_{1}=Z_{\beta} \times L_{2}(\Omega) ; \\
A_{1}\left[\begin{array}{l}
z_{1}^{n} \\
z_{2}^{n}
\end{array}\right] \longrightarrow w=\left[\begin{array}{l}
w_{1} \\
w_{2}
\end{array}\right] \text { in } Y_{1} \equiv Z_{\beta} \times L_{2}(\Omega) .
\end{gathered}
$$

We must show that $z \in \mathscr{D}\left(A_{1}\right)$ and $A_{1} z=w$. We rewrite (A.2c) explicitly via (2.12a), namely

$$
A_{1}\left[\begin{array}{l}
z_{1}^{n} \\
z_{2}^{n}
\end{array}\right]=\left[\begin{array}{c}
z_{2}^{n} \\
-A_{N}\left(z_{1}^{n}+\beta N_{0} D_{0} N_{0}^{*} A_{N} z_{1}^{n}+N_{0} D_{0} N_{0}^{*} A_{N} z_{2}^{n}\right)-d_{2} z_{2}^{n}
\end{array}\right] \longrightarrow\left[\begin{array}{l}
w_{1} \\
w_{2}
\end{array}\right]
$$

Without loss of generality, we may take $d_{2}=0$. From (A.2a) and (A.2b), it follows that $z_{1}^{n} \rightarrow z_{1}$ in $Z_{\beta}$, hence by (2.8) and (2.9),

$$
A_{N}^{1 / 2} z_{1}^{n} \longrightarrow A_{N}^{1 / 2} z_{1}, \quad D_{0}^{1 / 2} N_{0}^{*} A_{N} z_{1}^{n} \longrightarrow D_{0}^{1 / 2} N_{0}^{*} A_{N} z_{1} \text { in } L_{2}(\Omega) .
$$


Next, from (A.3), we have $z_{2}^{n} \rightarrow w_{1}$ in $Z_{\beta}$, which combined with (A.2b) implies via the $Z_{\beta}$-norm (2.9):

$$
z_{2} \equiv w_{1} \in Z_{\beta}, \quad A_{N}^{1 / 2} z_{2}^{n} \longrightarrow A_{N}^{1 / 2} z_{2}, \quad D_{0}^{1 / 2} N_{0}^{*} A_{N} z_{2}^{n} \longrightarrow D_{0}^{1 / 2} N_{0}^{*} A_{N} z_{2} \text { in } L_{2}(\Omega) .
$$

We set

$$
Q_{0} \equiv D_{0}^{1 / 2} N_{0}^{*} A_{N}^{1 / 2}: L_{2}(\Omega) \supset \mathscr{D}\left(Q_{0}\right) \longrightarrow L_{2}\left(\Gamma_{0}\right)
$$

We will show below that $\mathscr{D}\left(A_{N}^{1 / 4}\right) \subset \mathscr{D}\left(Q_{0}\right)$ by virtue of assumption $(1.5)$ on $D_{0}$, so that $Q_{0}$ is densely defined on $L_{2}(\Omega)$. Moreover, $Q_{0}=D_{0}^{1 / 2} N_{0}^{*} A_{N}^{1 / 2}$ is closed being the composition of the closed boundedly invertible operator $D_{0}^{1 / 2}$ —again as a consequence of (1.4) — with the bounded operator $N_{0}^{*} A_{N}^{1 / 2} \in \mathscr{L}\left(L_{2}(\Omega) ; L_{2}\left(\Gamma_{0}\right)\right)$ (see (2.4) and (2.5) (cf. [15, page 164])). Thus, the adjoint $Q_{0}^{*}=A_{N}^{1 / 2} N_{0} D_{0}^{1 / 2}$ is well defined and closed, as an operator $L_{2}\left(\Gamma_{0}\right) \supset \mathscr{D}\left(Q_{0}^{*}\right) \rightarrow L_{2}(\Omega)$. After these preliminaries, we multiply by $A_{N}^{-1 / 2}$ across the second line in (A.3) with $d_{2}=0$, and obtain with $w_{2} \in L_{2}(\Omega)$ and (A.3):

$$
\underbrace{A_{N}^{1 / 2} z_{1}^{n}}_{s_{1, n}}+\underbrace{\beta Q_{0}^{*} D_{0}^{1 / 2} N_{0}^{*} A_{N} z_{1}^{n}}_{s_{2, n}}+\underbrace{Q_{0}^{*} D_{0}^{1 / 2} N_{0}^{*} A_{N} z_{2}^{n}}_{s_{3, n}} \longrightarrow-A_{N}^{-1 / 2} w_{2} \text { in } L_{2}(\Omega) .
$$

Next, invoking the convergence properties in (A.4) (right), (A.5), we then obtain for the closed operator $Q_{0}^{*}$ via (A.7) that

$$
\begin{gathered}
D_{0}^{1 / 2} N_{0}^{*} A_{N} z_{1}^{n}, D_{0}^{1 / 2} N_{0}^{*} A_{N} z_{2}^{n} \in \mathscr{D}\left(Q_{0}^{*}\right), \\
s_{2, n}+s_{3, n} \longrightarrow \beta Q_{0}^{*} D_{0}^{1 / 2} N_{0}^{*} A_{N} z_{1}+Q_{0}^{*} D_{0}^{1 / 2} N_{0}^{*} A_{N} z_{2} .
\end{gathered}
$$

This then implies via (A.4) (left)

$$
A_{N}^{1 / 2} z_{1}+\beta Q_{0}^{*} D_{0}^{1 / 2} N_{0}^{*} A_{N} z_{1}+Q_{0}^{*} D_{0}^{1 / 2} N_{0}^{*} A_{N} z_{2}=-A_{N}^{-1 / 2} w_{2} \in L_{2}(\Omega),
$$

and therefore recalling $Q_{0}^{*}=A_{N}^{1 / 2} N_{0} D_{0}^{1 / 2}$ :

$$
\begin{gathered}
z_{1}+\beta N_{0} D_{0} N_{0}^{*} A_{N} z_{1}+N_{0} D_{0} N_{0}^{*} A_{N} z_{2} \in \mathscr{D}\left(A_{N}\right), \\
A_{N}\left(z_{1}+\beta N_{0} D_{0} N_{0}^{*} A_{N} z_{1}+N_{0} D_{0} N_{0}^{*} A_{N} z_{2}\right)=-w_{2} \in L_{2}(\Omega) .
\end{gathered}
$$

Thus, (A.4), (A.5), and (A.10) show $z \in \mathscr{D}\left(A_{1}\right)$ and $A_{1} z=w$, as desired.

It remains to show that $\mathscr{D}\left(A_{N}^{1 / 4}\right) \subset \mathscr{D}\left(Q_{0}\right)$ as claimed below (A.6), and so the operator $Q_{0}$ is densely defined on $L_{2}(\Omega)$. Indeed, let $\phi \in \mathscr{D}\left(A_{N}^{1 / 4}\right)$, so that $\psi=$ $A_{N}^{-1 / 2} \phi \in \mathscr{D}\left(A_{N}^{3 / 4}\right) \subset H^{3 / 2}(\Omega)$ and by (2.6) and trace theory,

$$
N_{0}^{*} A_{N}^{1 / 2} \phi=N_{0}^{*} A_{N} \psi=\left.\psi\right|_{\Gamma_{0}} \in H^{1}\left(\Gamma_{0}\right) .
$$


Consequently, by (A.6), $Q_{0} \phi \equiv D_{0}^{1 / 2} N_{0}^{*} A_{N}^{1 / 2} \phi=D_{0}^{1 / 2}\left(\left.\psi\right|_{\Gamma_{0}}\right) \in L_{2}\left(\Gamma_{0}\right)$, as desired, since $D_{0}^{1 / 2}$ is continuous from $H^{1}\left(\Gamma_{0}\right) \rightarrow L_{2}\left(\Gamma_{0}\right)$, as it follows from (1.5) of Hypothesis 1.3. Lemma 2.4 is proved.

Proof of Theorem 1.6. Under Assumptions 1.1, 1.2, and 1.3, we want to show that, for all $d_{2}, \beta \geq 0$, the operator $A$ defined by (2.37) and (2.38) is the generator of a strongly continuous semigroup $e^{A t}$ of contractions on the space $Y$, $t \geq 0$ defined in (2.10) or (2.36).

As in the previous Lemma 2.4, we likewise have that $A$ in (2.37) and (2.38) and the corresponding $Y$-adjoint $A^{*}$ are densely defined and dissipative on the space $Y$, due to the critical choice (2.8) and (2.9) of the norm of the component space $Z_{\beta}$. It is thus sufficient to show that $A$ is a closed operator. Let

$$
\begin{gathered}
\zeta^{n} \equiv\left[z_{1}^{n}, z_{2}^{n}, v_{1}^{n}, v_{2}^{n}\right] \in \mathscr{D}(A) \subset Z_{\beta} \times Z_{\beta} \times \mathscr{D}\left(\mathscr{A}^{1 / 2}\right) \times \mathscr{D}\left(\mathscr{A}^{1 / 2}\right) ; \\
\zeta^{n} \longrightarrow \zeta=\left[z_{1}, z_{2}, v_{1}, v_{2}\right] \text { in } Y ; \\
A \zeta^{n} \longrightarrow l=\left[l_{1}, l_{2}, l_{3}, l_{4}\right] \text { in } Y=Z_{\beta} \times L_{2}(\Omega) \times \mathscr{D}\left(\mathscr{A}^{1 / 2}\right) \times L_{2}\left(\Gamma_{0}\right) .
\end{gathered}
$$

We must show that $\zeta \in \mathscr{D}(A)$ and $A \zeta=l$. Explicitly, (A.12) reads via (2.37) and (2.38) as

$$
\left[\begin{array}{c}
z_{2}^{n} \\
-A_{N}\left(z_{1}^{n}+\beta N_{0} D_{0} N_{0}^{*} A_{N} z_{1}^{n}+N_{0} D_{0} N_{0}^{*} A_{N} z_{2}^{n}\right)-d_{2} z_{2}^{n}+A_{N} N_{0} v_{2}^{n} \\
v_{2}^{n} \\
-N_{0}^{*} A_{N} z_{2}^{n}-\mathscr{A} v_{1}^{n}-\rho \mathscr{A}^{\alpha} v_{2}^{n}
\end{array}\right] \rightarrow\left[\begin{array}{l}
l_{1} \\
l_{2} \\
l_{3} \\
l_{4}
\end{array}\right]
$$

Again, without loss of generality we will set $d_{2}=0$. From (A.13) it follows that $z_{2}^{n} \rightarrow l_{1}$ in $Z_{\beta}$, which combined with (A.12) yields via the $Z_{\beta}$-norm (2.9),

$$
\begin{gathered}
z_{2}=l_{1} \in Z_{\beta}, \quad A_{N}^{1 / 2} z_{2}^{n} \longrightarrow A_{N}^{1 / 2} z_{2}, \\
D_{0}^{1 / 2} N_{0}^{*} A_{N} z_{2}^{n} \longrightarrow D_{0}^{1 / 2} N_{0}^{*} A_{N} z_{2} \text { in } L_{2}(\Omega) .
\end{gathered}
$$

Analogously, from (A.13) it also follows that $v_{2}^{n} \rightarrow l_{3}$ in $\mathscr{D}\left(\mathscr{A}^{1 / 2}\right)$, which combined with (A.12) yields

$$
l_{3}=v_{2} \in \mathscr{D}\left(\mathscr{A}^{1 / 2}\right), \quad \mathscr{A}^{1 / 2} v_{2}^{n} \longrightarrow \mathscr{A}^{1 / 2} v_{2} \text { in } L_{2}\left(\Gamma_{0}\right) .
$$

Next, proceeding as in the preceding proof of Lemma 2.4, we deduce first that ( for $\left.d_{2}=0\right)$ :

$$
\begin{aligned}
A_{N}^{1 / 2} z_{1}^{n} & +\beta Q_{0}^{*} D_{0}^{1 / 2} N_{0}^{*} A_{N} z_{1}^{n}+Q_{0}^{*} D_{0}^{1 / 2} N_{0}^{*} A_{N} z_{2}^{n}-A_{N}^{1 / 2} N_{0} v_{2}^{n} \\
& \longrightarrow A_{N}^{1 / 2} z_{1}+\beta Q_{0}^{*} D_{0}^{1 / 2} N_{0}^{*} A_{N} z_{1}+Q_{0}^{*} D_{0}^{1 / 2} N_{0}^{*} A_{N} z_{2}-A_{N}^{1 / 2} N_{0} v_{2} \\
& =-A_{N}^{-1 / 2} l_{2},
\end{aligned}
$$


so that by (A.16) and $Q_{0}^{*}=A_{N}^{1 / 2} N_{0} D_{0}^{1 / 2}$,

$$
\begin{aligned}
& z_{1}+\beta N_{0} D_{0} N_{0}^{*} A_{N} z_{1}+N_{0} D_{0} N_{0}^{*} A_{N} z_{2}-N_{0} v_{2} \in \mathscr{D}\left(A_{N}\right), \\
& A_{N}\left[z_{1}+\beta N_{0} D_{0} N_{0}^{*} A_{N} z_{1}+N_{0} D_{0} N_{0}^{*} A_{N} z_{2}-N_{0} v_{2}\right]=-l_{2} .
\end{aligned}
$$

Finally, we consider the last convergence statement in (A.13), namely that

$$
-N_{0}^{*} A_{N} z_{2}^{n}-\mathscr{A} v_{1}^{n}-\rho \mathscr{A}^{\alpha} v_{2}^{n} \longrightarrow l_{4}
$$

We have already seen in (A.14) that $A_{N}^{1 / 2} z_{2}^{n} \rightarrow A_{N}^{1 / 2} z_{2}$ in $L_{2}(\Omega)$, and since $N_{0}^{*} A_{N}^{1 / 2}$ is bounded by (2.5), then it follows that

$$
N_{0}^{*} A_{N} z_{2}^{n} \longrightarrow N_{0}^{*} A_{N} z_{2} \text { in } L_{2}(\Omega) .
$$

We also have seen in (A.15) that $\mathscr{A}^{1 / 2} v_{2}^{n} \rightarrow \mathscr{A}^{1 / 2} v_{2}$ in $L_{2}\left(\Gamma_{0}\right)$, while $\mathscr{A}^{1 / 2} v_{1}^{n} \rightarrow$ $\mathscr{A}^{1 / 2} v_{1}$ in $L_{2}\left(\Gamma_{0}\right)$ holds by (A.12). Hence, since $1-\alpha \leq 1 / 2$ by assumption, then

$$
\mathscr{A}^{1-\alpha} v_{1}^{n}+\rho v_{2}^{n} \longrightarrow \mathscr{A}^{1-\alpha} v_{1}+\rho v_{2} \text { in } L_{2}\left(\Gamma_{0}\right)
$$

Note that by (A.12) — recalling the definition of $\mathscr{D}(A)$ in (2.38) —we have $\mathscr{A}^{1-\alpha} v_{1}^{n}$ $+\rho v_{2}^{n} \in \mathscr{D}\left(\mathscr{A}^{\alpha}\right)$. Furthermore, (A.18) and (A.20) imply since $z_{2}=\ell_{1}$ in (A.14):

$$
-\mathscr{A} v_{1}^{n}-\rho \mathscr{A}^{\alpha} v_{2}^{n}=-\mathscr{A}^{\alpha}\left(\mathscr{A}^{1-\alpha} v_{1}^{n}+\rho v_{2}^{n}\right) \longrightarrow l_{4}+N_{0}^{*} A_{N} l_{1}
$$

Therefore, since $\mathscr{A}^{\alpha}$ is closed, it follows from (A.21) that $\mathscr{A}^{1-\alpha} v_{1}+\rho v_{2} \in \mathscr{D}\left(\mathscr{A}^{\alpha}\right)$ and $-N_{0}^{*} A_{N} z_{2}-\mathscr{A} v_{1}-\rho \mathscr{A}^{\alpha} v_{2}=l_{4}$, which concludes the proof.

\section{B. Second proof of Lemma 3.3}

Proof of estimate (3.11) for $\alpha-2 r \geq-2 r_{0}$

Step 1. Our starting point is again (3.2b), which this time, however, we rewrite as

$$
\left[\begin{array}{c}
v(t) \\
v_{t}(t)
\end{array}\right]=e^{A_{2} t}\left[\begin{array}{c}
0 \\
v_{2}
\end{array}\right]-\int_{0}^{t} e^{A_{2}(t-\tau)}\left[\begin{array}{c}
0 \\
N_{0}^{*} A_{N} z_{\tau}(\tau)
\end{array}\right] d \tau
$$

in view of (2.13) (see also (3.26) and (3.27)). We next proceed as in the proof of Proposition 3.5, the part that follows (3.27), except that we work with different exponents.

Step 2. We will show that there exists a constant $C_{T}$ such that

$$
\begin{aligned}
\left\|\mathscr{A}^{-r_{0}} v_{t}(t)\right\|_{L_{2}\left(\Gamma_{0}\right)}^{2} \leq & c_{T}\left[\left\|A_{2}^{-r_{0} / \alpha} e^{A_{2} t}\left[\begin{array}{c}
0 \\
v_{2}
\end{array}\right]\right\|_{Y_{2}}^{2}+\left\|z_{0}\right\|_{Z_{\beta}}^{2}\right] \\
& +C_{T} \int_{0}^{t}\left\|\mathscr{A}^{-r_{0}} v_{\tau}(\tau)\right\|_{L_{2}\left(\Gamma_{0}\right)}^{2} d \tau, \quad 0 \leq t \leq T .
\end{aligned}
$$


Proof of (B.2). We apply $A_{2}^{-r_{0} / \alpha}$ to (B.1) and obtain

$$
A_{2}^{-r_{0} / \alpha}\left[\begin{array}{c}
v(t) \\
v_{t}(t)
\end{array}\right]=A_{2}^{-r_{0} / \alpha} e^{A_{2} t}\left[\begin{array}{c}
0 \\
v_{2}
\end{array}\right]-\int_{0}^{t} A_{2}^{-2 r_{0} / \alpha} e^{A_{2}(t-\tau)} A_{2}^{r_{0} / \alpha}\left[\begin{array}{c}
0 \\
N_{0}^{*} A_{N} z_{\tau}(\tau)
\end{array}\right] d \tau
$$

From (B.3), recalling (2.25) with $s=r_{0} / \alpha \leq 1 / 2$ (since $r_{0} \leq 1 / 4$ by Assumption 1.3 , and $1 / 2 \leq \alpha)$, we in turn deduce that

$$
\begin{aligned}
\left\|\mathscr{A}^{-r_{0}} v_{t}(t)\right\|_{L_{2}\left(\Gamma_{0}\right)}^{2} \leq & \left\|A_{2}^{-r_{0} / \alpha}\left[\begin{array}{c}
v(t) \\
v_{t}(t)
\end{array}\right]\right\|_{Y_{2}}^{2} \leq 2\left\|A_{2}^{-r_{0} / \alpha} e^{A_{2} t}\left[\begin{array}{c}
0 \\
v_{2}
\end{array}\right]\right\|_{Y_{2}}^{2} \\
& +2 T^{2} \int_{0}^{t}\left\|A_{2}^{-2 r_{0} / \alpha} e^{A_{2}(t-\tau)} A_{2}^{r_{0} / \alpha}\left[\begin{array}{c}
0 \\
N_{0}^{*} A_{N} z_{\tau}(\tau)
\end{array}\right]\right\|_{Y_{2}}^{2} d \tau \\
\leq & 2\left\|A_{2}^{-r_{0} / \alpha} e^{A_{2} t}\left[\begin{array}{c}
0 \\
v_{2}
\end{array}\right]\right\|_{Y_{2}}^{2}+c_{T} \int_{0}^{t}\left\|A_{2}^{r_{0} / \alpha}\left[\begin{array}{c}
0 \\
N_{0}^{*} A_{N} z_{\tau}(\tau)
\end{array}\right]\right\|_{Y_{2}}^{2} d \tau,
\end{aligned}
$$

after using the Schwarz inequality on the integral term of (B.3). Recalling this time (2.23) with $s=r_{0} / \alpha \leq 1 / 2$ in the integrand term of (B.4), we then obtain from (B.4),

$$
\begin{aligned}
\left\|\mathscr{A}^{-r_{0}} v_{t}(t)\right\|_{L_{2}\left(\Gamma_{0}\right)}^{2} \leq & 2\left\|A_{2}^{-r_{0} / \alpha} e^{A_{2} t}\left[\begin{array}{c}
0 \\
v_{2}
\end{array}\right]\right\|_{Y_{2}}^{2} \\
& +c_{T} \int_{0}^{t}\left\|\mathscr{A}^{r_{0}} N_{0}^{*} A_{N} z_{\tau}(\tau)\right\|_{L_{2}\left(\Gamma_{0}\right)}^{2} d \tau \\
(\text { by }(3.10)) \leq & {\left[2\left\|A_{2}^{-r_{0} / \alpha} e^{A_{2} t}\left[\begin{array}{c}
0 \\
v_{2}
\end{array}\right]\right\|_{Y_{2}}^{2}+c_{T}\left\|z_{0}\right\|_{Z_{\beta}}^{2}\right] } \\
& +C_{T} \int_{0}^{t}\left\|\mathscr{A}^{-r_{0}} v_{\tau}(\tau)\right\|_{L_{2}\left(\Gamma_{0}\right)}^{2} d \tau,
\end{aligned}
$$

where in the last step we have used estimate (3.10). Then, (B.6) proves estimate (B.2), as desired.

Step 3. Integrating inequality (B.2) in $t$ yields

$$
\begin{aligned}
\int_{0}^{t}\left\|\mathscr{A}^{-r_{0}} v_{\tau}(\tau)\right\|_{L_{2}\left(\Gamma_{0}\right)}^{2} d \tau \leq & \left\{c_{T} \int_{0}^{t}\left\|A_{2}^{-r_{0} / \alpha} e^{A_{2} \tau}\left[\begin{array}{c}
0 \\
v_{2}
\end{array}\right]\right\|_{Y_{2}}^{2}+c_{T} t\left\|z_{0}\right\|_{Z_{\beta}}^{2}\right\} \\
& +C_{T} \int_{0}^{t} \int_{0}^{\tau}\left\|\mathscr{A}^{-r_{0}} v_{s}(s)\right\|_{L_{2}\left(\Gamma_{0}\right)}^{2} d s d \tau .
\end{aligned}
$$

Applying now the Gronwall inequality [37, Lemma 2.1, page 92] to inequality (B.7) for the unknown

$$
F(t)=\int_{0}^{t}\left\|\mathscr{A}^{-r_{0}} v_{\tau}(\tau)\right\|_{L_{2}\left(\Gamma_{0}\right)}^{2} d \tau
$$


(notice that the assumption in [37, Lemma 2.1] that the function $\ell(t)=\operatorname{term}\{\}$ in (B.7) be nondecreasing is satisfied), or else [29, page 205], we obtain

$$
\begin{aligned}
F(t) & =\int_{0}^{t}\left\|\mathscr{A}^{-r_{0}} v_{\tau}(\tau)\right\|_{L_{2}\left(\Gamma_{0}\right)}^{2} d \tau \\
& \leq e^{C_{T} t}\left\{c_{T} \int_{0}^{t}\left\|A_{2}^{-r_{0} / \alpha} e^{A_{2} \tau}\left[\begin{array}{c}
0 \\
v_{2}
\end{array}\right]\right\|_{Y_{2}}^{2}+c_{T} t\left\|z_{0}\right\|_{Z_{\beta}}^{2}\right\} .
\end{aligned}
$$

Step 4. We now claim that the following estimate holds true for any $0<T_{0}<$ $\infty$ :

$$
\int_{0}^{T_{0}}\left\|\left(-A_{2}\right)^{s} e^{A_{2} t} y_{2}\right\|_{Y_{2}}^{2} \leq C_{T_{0}}\left\|y_{2}\right\|_{Y_{2}}^{2}, \quad 0 \leq s \leq \frac{1}{2} .
$$

Indeed, if $s=1 / 2-\epsilon, \epsilon>0$, estimate (B.10) trivially follows from the analyticity estimate $\left\|\left(-A_{2}\right)^{1 / 2-\epsilon} \exp \left(A_{2} t\right)\right\| \leq C / t^{1 / 2-\epsilon}, 0<t \leq T_{0}$ guaranteed by the property of Lemma 2.7 that $e^{A_{2} t}$ is an s.c. analytic semigroup on $Y_{2}$ (which, moreover, is uniformly (exponentially) stable here). To push the validity of (B.10) to the case $\epsilon=0$, or $s=1 / 2$, we use, from [6, Appendix A], stronger spectral properties enjoyed by $A_{2}$. These, in turn, provide an explicit spectral representation of $e^{A_{2} t}$ which a fortiori shows that $e^{A_{2} t}$ is analytic and uniformly stable. Indeed, it turns out that $A_{2}$ is, in fact, the direct (nonorthogonal) sum of two normal operators on $Y_{2}$ except for some exceptional values of $\rho, \alpha$, where then $A_{2}$ is still a spectral operator. As a consequence of the explicit spectral expansion representation of the two components of $e^{A_{2} t}$, it follows that estimate (B.10) holds true in the case $s=1 / 2$ as well. ([6, Appendix A] is written explicitly in the case where $A_{2}$ has compact resolvent, but this property can be dispensed with.) The Claim is established.

Next, we apply estimate (B.10) for the first term on the right-hand side of (B.9), with $s=\left(r-r_{0}\right) / \alpha \leq 1 / 2$ as assumed in the statement of Lemma 3.3, to obtain

$$
\begin{aligned}
\int_{0}^{t}\left\|A_{2}^{-r_{0} / \alpha} e^{A_{2} \tau}\left[\begin{array}{c}
0 \\
v_{2}
\end{array}\right]\right\|_{Y_{2}}^{2} d \tau & \leq \int_{0}^{T}\left\|\left(-A_{2}\right)^{\left(r-r_{0}\right) / \alpha} e^{A_{2} t}\left(-A_{2}\right)^{-r / \alpha}\left[\begin{array}{c}
0 \\
v_{2}
\end{array}\right]\right\|_{Y_{2}}^{2} d t \\
& \leq C_{T}\left\|A_{2}^{-r / \alpha}\left[\begin{array}{c}
0 \\
v_{2}
\end{array}\right]\right\|_{Y_{2}}^{2}=C_{T}\left\|\mathscr{A}^{-r} v_{2}\right\|_{L_{2}\left(\Gamma_{0}\right)}^{2},
\end{aligned}
$$

where in the last step we have also used (2.26). Substituting (B.11) in the first term on the right-hand side of (B.9) yields

$$
\int_{0}^{t}\left\|\mathscr{A}^{-r_{0}} v_{\tau}(\tau)\right\|_{L_{2}\left(\Gamma_{0}\right)}^{2} d \tau \leq c_{T} e^{C_{T} t}\left[\left\|\mathscr{A}^{-r} v_{2}\right\|_{L_{2}\left(\Gamma_{0}\right)}^{2}+\left\|z_{0}\right\|_{Z_{\beta}}^{2}\right],
$$

and (B.12) proves estimates (3.11), as desired. 
Remark B.1. If $\left(r-r_{0}\right) / \alpha<1 / 2$ (i.e., if we exclude the limit case $\left.\left(r-r_{0}\right) / \alpha=1 / 2\right)$, then the following alternative proof may be given. Rewrite and estimate, just by analyticity, the first term on the right-hand side of (B.6) as follows:

$$
\begin{aligned}
\left\|A_{2}^{-r_{0} / \alpha} e^{A_{2} t}\left[\begin{array}{c}
0 \\
v_{2}
\end{array}\right]\right\|_{Y_{2}} & =\int_{0}^{T}\left\|A_{2}^{\left(r-r_{0}\right) / \alpha} e^{A_{2} t} A_{2}^{-r / \alpha}\left[\begin{array}{c}
0 \\
v_{2}
\end{array}\right]\right\|_{Y_{2}} d \tau \\
& \leq \frac{C_{T}}{t^{\left(r-r_{0}\right) / \alpha}}\left\|A_{2}^{-r / \alpha}\left[\begin{array}{c}
0 \\
v_{2}
\end{array}\right]\right\|_{Y_{2}} \leq \frac{C_{T}}{t^{\left(r-r_{0}\right) / \alpha}}\left\|\mathscr{A}^{-r} v_{2}\right\|_{L_{2}\left(\Gamma_{0}\right)},
\end{aligned}
$$

where, in the last step, we have used (2.26) with $s=\left(r-r_{0}\right) / \alpha \leq 1 / 2$ (at this stage we could allow also the limit case $s=1 / 2$ ). We now use (B.13) in (B.6) and obtain

$$
\begin{aligned}
\left\|\mathscr{A}^{-r_{0}} v_{2}\right\|_{L_{2}\left(\Gamma_{0}\right)}^{2} \leq & \frac{C_{T}}{t^{2 s}}\left\|\mathscr{A}^{-r} v_{2}\right\|_{L_{2}\left(\Gamma_{0}\right)}^{2}+c_{T}\left\|A_{N}^{1 / 2} z_{0}\right\|_{L_{2}(\Omega)}^{2} \\
& +c_{T} \int_{0}^{t}\left\|\mathscr{A}^{-r_{0}} v_{\tau}(\tau)\right\|_{L_{2}\left(\Gamma_{0}\right)}^{2} d \tau
\end{aligned}
$$

with $s=\left(r-r_{0}\right) / \alpha \leq 1 / 2$. We now apply the Gronwall's inequality for $2 s<1$ (critical) as in [12, Section 1.2.1, page 6], with $\alpha=2 s<1, \beta=0$ to obtain

$$
\left\|\mathscr{A}^{-r_{0}} v_{t}(t)\right\|_{L_{2}\left(\Gamma_{0}\right)}^{2} \leq \frac{C_{T}}{t^{2 s}}\left\|\mathscr{A}^{-r} v_{2}\right\|_{L_{2}\left(\Gamma_{0}\right)}^{2}+c_{T}^{\prime}\left\|A_{N}^{1 / 2} z_{0}\right\|_{L_{2}(\Omega)}^{2} .
$$

Finally from (B.15), using again critically that $s=\left(r-r_{0}\right) / \alpha<1 / 2$, we obtain

$$
\begin{aligned}
\int_{0}^{t}\left\|\mathscr{A}^{-r_{0}} v_{\tau}(\tau)\right\|_{L_{2}\left(\Gamma_{0}\right)}^{2} d \tau & \leq C_{T}\left(\int_{0}^{t} \frac{1}{\tau^{2 s}} d \tau\right)\left\|\mathscr{A}^{-r} v_{2}\right\|_{L_{2}\left(\Gamma_{0}\right)}^{2}+c_{T}^{\prime}\left\|A_{N}^{1 / 2} z_{0}\right\|_{L_{2}(\Omega)}^{2} \\
& \leq \frac{C_{T}}{1-2 s} t^{1-2 s}\left[\left\|\mathscr{A}^{-r} v_{2}\right\|_{L_{2}\left(\Gamma_{0}\right)}^{2}+\left\|A_{N}^{1 / 2} z_{0}\right\|_{L_{2}(\Omega)}^{2}\right],
\end{aligned}
$$

with $1-2 s>0$, thus re-proving $($ B.12) $=(3.11)$.

C. Numerical graphs of the spectrum of the operator $A$ in (2.37) and (2.38) with $\alpha=1 / 2, \beta=0$, when $\Omega$ is a 2-dimensional square. Contrast between the case $D_{0}=-d^{2} / d x^{2}$ and the case $D_{0}=0$ along an edge of the square

At our request, the numerical graphs below were kindly computed in the spring of 2001 by Catherine Lebiedzik while at the University of Virginia. We wish to thank her for permission to include them here. These graphs are part of a larger collection of graphs, computed by her, which refer to various combinations of the acoustic model (1.2). Individually and cumulatively, these graphs support the theoretical conclusions of the present paper.

Case $D_{0}=-d^{2} / d x^{2}$ along an edge: Figures C.1 and C.2. Let $\Omega$ be a 2-dimensional square, as indicated below. Figures C. 1 and C. 2 refer to the (static) operator $A$ in 
232 Singular estimates and uniform stability

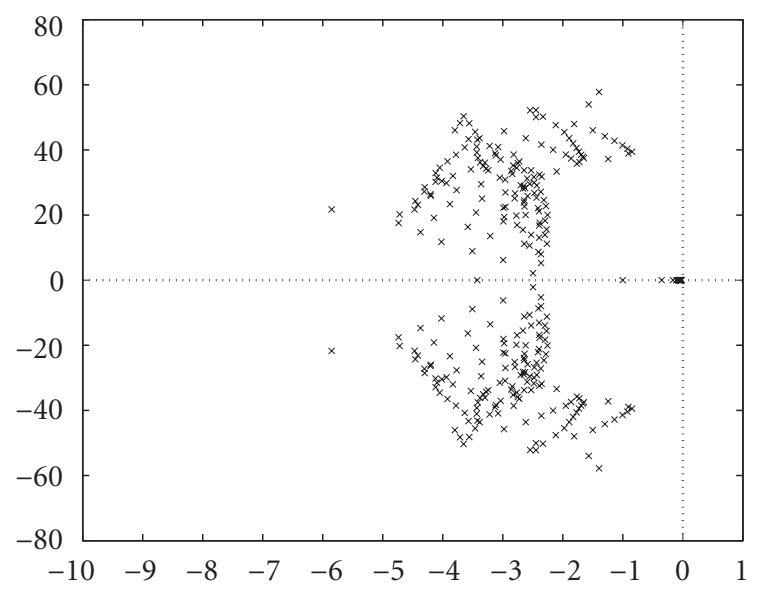

Figure C.1. Viscous damping, $d_{2}=1 ; D_{0}=-d^{2} / d x^{2}$.

(2.37) and (2.38), corresponding to the following structural acoustic problem:

$$
\begin{array}{ll}
z_{t t}=\Delta z-d_{2} z_{t} & \text { in }(0, T] \times \Omega, \\
\frac{\partial z}{\partial v}+z=0 & \text { on }(0, T] \times \Gamma_{1}, \\
\frac{\partial z}{\partial v}-\frac{d^{2}}{d x^{2}} z_{t}-v_{t}=0 & \text { on }(0, T] \times \Gamma_{0}, \\
v_{t t}+\Delta^{2} v-\Delta v_{t}+z_{t}=0 & \text { on }(0, T] \times \Gamma_{0}, \\
v=\Delta v=0 & \text { on }(0, T] \times \partial \Gamma_{0},
\end{array}
$$

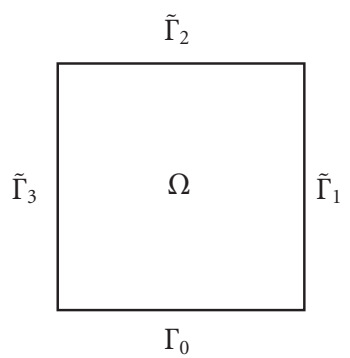

where $\Gamma_{1}=\tilde{\Gamma}_{1} \cup \tilde{\Gamma}_{2} \cup \tilde{\Gamma}_{3}$, and $d=1$. This problem corresponds to the case $\alpha=1 / 2$, $\beta=0$, and $D_{0}=-d^{2} / d x^{2}$ in model (1.1). Figure C.1 and its expanded version, Figure C.2, on an enlarged scale show two main branches of eigenvalues of $A$ : one corresponding to the parabolic component and one corresponding to the hyperbolic component. In particular, the sub-branch of eigenvalues on the negative real axis appear to accumulate to the origin $\lambda=0$. From Proposition 4.3(i), 
F. Bucci et al. 233

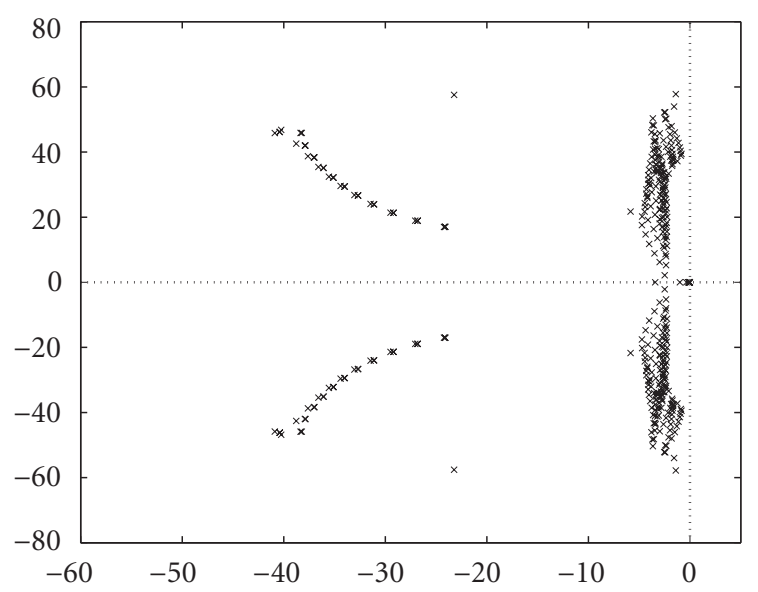

Figure C.2. Viscous damping, $d_{2}=1 ; D_{0}=-d^{2} / d x^{2}$.

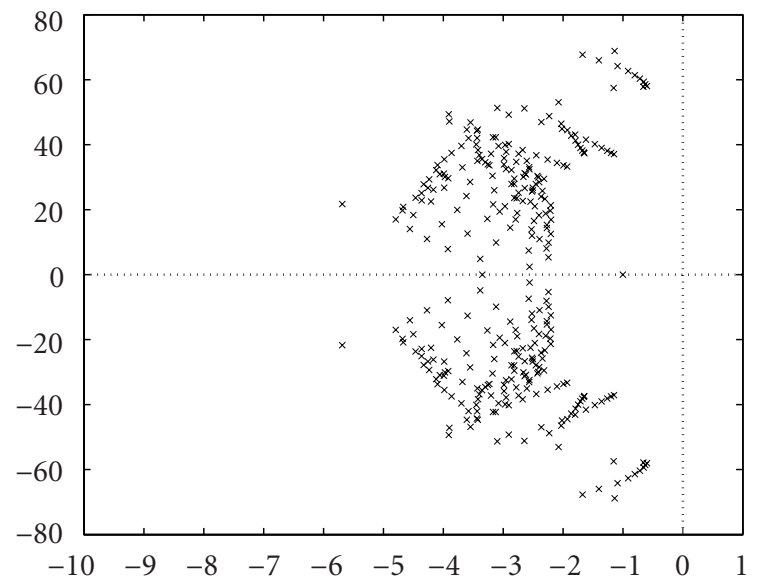

Figure C.3. Viscous damping, $d_{2}=1 ; D_{0}=0$.

(4.12), combined with Remark 4.4, we know that $\lambda=0$ is a point (unique) of the continuous spectrum of ( $A_{1}$ and) $A$, since $D_{0}$ is presently of order $s=2$.

On the other hand, the spectrum of $A$ outside the real axis appears bounded away from the imaginary axis; more precisely on the left of the vertical line $\operatorname{Re} \lambda=$ $-d / 2=-1 / 2$, in agreement with Proposition 4.5(i), (4.32).

Case $D_{0}=0$ along an edge: Figures C.3 and C.4. Let $\Omega$ be a 2-dimensional square, as indicated below. Figures C. 3 and C.4 refer to the static operator $A$ in (2.37) 
234 Singular estimates and uniform stability

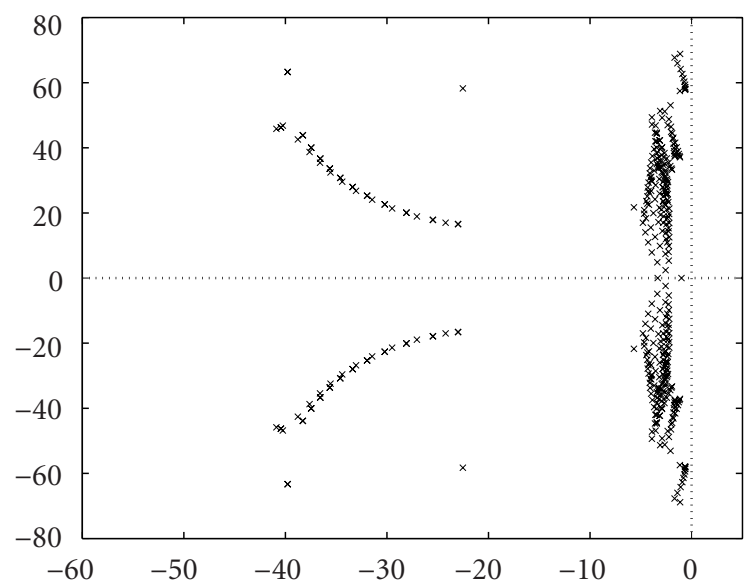

Figure C.4. Viscous damping, $d_{2}=1 ; D_{0}=0$.

and (2.38), corresponding to the following structural acoustic problem:

$$
\begin{array}{ll}
z_{t t}=\Delta z-d_{2} z_{t} & \text { in }(0, T] \times \Omega, \\
\frac{\partial z}{\partial v}+z=0 & \text { on }(0, T] \times \Gamma_{1}, \\
\frac{\partial z}{\partial v}-v_{t}=0 & \text { on }(0, T] \times \Gamma_{0}, \\
v_{t t}+\Delta^{2} v-\Delta v_{t}+z_{t}=0 & \text { on }(0, T] \times \Gamma_{0}, \\
v=\Delta v=0 & \text { on }(0, T] \times \partial \Gamma_{0},
\end{array}
$$

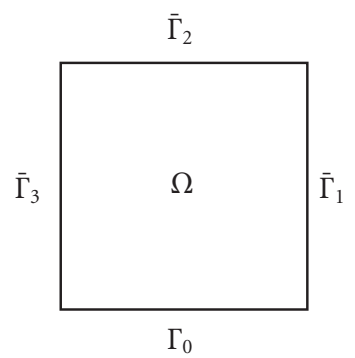

where $\Gamma_{1}=\tilde{\Gamma}_{1} \cup \tilde{\Gamma}_{2} \cup \tilde{\Gamma}_{3}$, and $d=1$. This problem corresponds to the case $\alpha=1 / 2$, $\beta=0$, and $D_{0}=0$ in model (1.1). Figure C.3 and its expanded version, Figure C.4, on an enlarged scale share with Figures C.1 and C.2 the same macroscopic pattern of the eigenvalues of $A$ (with two main branches: one corresponding to the parabolic component and one corresponding to the hyperbolic component), except for one main difference: now, with $D_{0}=0$, the sub-branch of eigenvalues on the negative real axis does not appear to converge to the origin, in line 
with Proposition 4.5(iii), (4.37). Moreover, $\lambda=0$ is the resolvent set of $\left(A_{1}\right.$ and of) $A$ in this case, in line with Proposition 4.3(i), (4.11). On the other hand, as for Figures C.1 and C.2, the spectrum outside the real axis appears bounded away from the imaginary axis; more precisely, on the left of the vertical line $\operatorname{Re} \lambda=-d / 2=-1 / 2$, in agreement with Proposition 4.5(i), (4.32).

\section{Acknowledgments}

Bucci's research performed while this author was visiting the University of Virginia, Mathematics Department, under a NATO-CNR senior fellowship.

Triggiani's research partially supported by the National Science Foundation (NSF) under grant DMS-9804056, and by the Army Research Office, under grant AAH04-96-1-0059.

\section{References}

[1] G. Avalos, Optimal control for a coupled hyperbolic-parabolic system in arising structural acoustics, Ph.D. thesis, University of Virginia, 1995.

[2] Sharp regularity estimates for solutions of the wave equation and their traces with prescribed Neumann data, Appl. Math. Optim. 35 (1997), no. 2, 203-219.

[3] G. Avalos and I. Lasiecka, Differential Riccati equation for the active control of a problem in structural acoustics, J. Optim. Theory Appl. 91 (1996), no. 3, 695-728.

[4] A. Bensoussan, G. Da Prato, M. C. Delfour, and S. K. Mitter, Representation and Control of Infinite-Dimensional Systems, Vol. II, Birkhäuser, Massachusetts, 1993.

[5] S. P. Chen and R. Triggiani, Proof of two conjectures of G. Chen and D. Russell on structural damping for elastic systems: The case $\alpha=\frac{1}{2}$, Proceedings of the Seminar on Approximation and Optimization (Cuba, 1987), Lecture Notes in Mathematics, vol. 1354, Springer-Verlag, 1987, pp. 234-256.

[6] Proof of extensions of two conjectures on structural damping for elastic systems, Pacific J. Math. 136 (1989), no. 1, 15-55.

[7] Characterization of domains of fractional powers of certain operators arising in elastic systems, and applications, J. Differential Equations 88 (1990), no. 2, 279293.

[8] E. K. Dimitriadis, C. R. Fuller, and C. A. Rogers, Piezoelectric actuators for distributed vibration excitation of thin plates, J. Vibration and Acoustic 113 (1991), 100-107.

[9] L. Gearhart, Spectral theory for contraction semigroups on Hilbert space, Trans. Amer. Math. Soc. 236 (1978), 385-394.

[10] P. Grisvard, Caractérisation de quelques espaces d'interpolation, Arch. Rational Mech. Anal. 25 (1967), 40-63 (French).

[11] _ Elliptic Problems in Nonsmooth Domains, Monographs and Studies in Mathematics, vol. 24, Pitman, Massachusetts, 1985.

[12] D. Henry, Geometric Theory of Semilinear Parabolic Equations, Lecture Notes in Mathematics, vol. 840, Springer-Verlag, Berlin, 1981.

[13] I. Herbst, The spectrum of Hilbert space semigroups, J. Operator Theory 10 (1983), no. 1, 87-94.

[14] J. S. Howland, On a theorem of Gearhart, Integral Equations Operator Theory 7 (1984), no. 1, 138-142.

[15] T. Kato, Perturbation Theory for Linear Operators, Springer-Verlag, New York, 1966. 
[16] I. Lasiecka, Mathematical Control Theory of Coupled PDE Systems, NSF-CMBS Lecture Notes, SIAM, Pennsylvania, 2001.

[17] I. Lasiecka, J.-L. Lions, and R. Triggiani, Nonhomogeneous boundary value problems for second order hyperbolic operators, J. Math. Pures Appl. (9) 65 (1986), no. 2, 149-192.

[18] I. Lasiecka and R. Triggiani, Optimal control and differential Riccati equations under singular estimates for $e^{A t} B$ in the absence of analyticity, to appear in Advances in Dynamics and Control.

[19] - Regularity of hyperbolic equations under $L_{2}\left(0, T ; L_{2}(\Gamma)\right)$-Dirichlet boundary terms, Appl. Math. Optim. 10 (1983), no. 3, 275-286.

[20] - Trace regularity of the solutions of the wave equation with homogeneous Neumann boundary conditions and data supported away from the boundary, J. Math. Anal. Appl. 141 (1989), no. 1, 49-71.

[21] Sharp regularity theory for second order hyperbolic equations of Neumann type. I. $L_{2}$ nonhomogeneous data, Ann. Mat. Pura Appl. (4) 157 (1990), 285-367.

[22] , Regularity theory of hyperbolic equations with nonhomogeneous Neumann boundary conditions. II. General boundary data, J. Differential Equations 94 (1991), no. 1, 112-164.

[23] _ Recent Advances in Regularity of Second Order Hyperbolic Mixed Problems, and Applications. Dynamics Reported, Expositions in Dynamical Systems. New Series, vol. 3, Springer-Verlag, Berlin, 1994.

[24] Control Theory for Partial Differential Equations: Continuous and Approximation Theories. Vol. I: Abstract Parabolic Systems, Cambridge University Press, Cambridge, 2000.

[25] _ Control Theory for Partial Differential Equations: Continuous and Approximation Theories. Vol. II: Abstract Hyperbolic-like Systems over a Finite Time Horizon, Cambridge University Press, Cambridge, 2000.

[26] Optimal control and algebraic Riccati equations under singular estimates for $e^{A t} B$ in the absence of analyticity, Differential Equations and Optimal Control, Marcel Dekker Lecture Notes in Pure and Applied Mathematics, vol. 225, Marcel Dekker, 2001, pp. 193-220.

[27] J.-L. Lions and E. Magenes, Non-Homogeneous Boundary Value Problems and Applications. Vol. I, Springer-Verlag, New York, 1972.

[28] , Non-Homogeneous Boundary Value Problems and Applications. Vol. II, Springer-Verlag, New York, 1972.

[29] R. H. Martin, Jr., Nonlinear Operators and Differential Equations in Banach Spaces, Pure and Applied Mathematics, Wiley \& Sons, New York, 1976.

[30] L. Monauni, Exponential decay of solutions to Cauchy's abstract problem as determined by the extended spectrum of the dynamical operator, Tech. Report LIDS-P-947, Department of Electrical Engineering, Massachusetts Institute of Technology, USA, 1979, Tech. Report No. 90, Control Theory Center, University of Warwick, England, 1980.

[31] P. M. Morse and K. Ingard, Theoretical Acoustics, McGraw-Hill, New York, 1968.

[32] A. Pazy, Semigroups of Linear Operators and Applications to Partial Differential Equations, Applied Mathematical Sciences, vol. 44, Springer-Verlag, New York, 1983.

[33] J. Prüss, On the spectrum of $C_{0}$-semigroups, Trans. Amer. Math. Soc. 284 (1984), no. 2, 847-857.

[34] D. Tataru, On the regularity of boundary traces for the wave equation, Ann. Scuola Norm. Sup. Pisa Cl. Sci. (4) 26 (1998), no. 1, 185-206. 
[35] R. Triggiani, Min-Max game theory and optimal control with indefinite cost under a singular estimate for $e^{A t} B$ in the absence of analyticity, invited paper in special volume of Birkhäuser Verlag in memory of B. Terreni; A. Lorenzi, and B. Ruf, eds., to appear.

[36] _ Regularity with interior point control. II. Kirchhoff equations, J. Differential Equations 103 (1993), no. 2, 394-420.

[37] J. Zabczyk, Mathematical Control Theory: An Introduction, Birkhäuser, Massachusetts, 1992.

F. Bucci: Dipartimento di Matematica Applicata “G. Sansone," Università degli Studi di Firenze, Via S. Marta 3, 50139 Firenze, Italy

E-mail address: fbucci@dma.unifi.it

I. Lasiecka: Department of Mathematics, University of Virginia, P.O. Box 400137, Charlottesville, VA 22904-4137, USA

E-mail address: il2v@virginia.edu

R. Triggiani: Department of Mathematics, University of Virginia, P.O. Box 400137, Charlottesville, VA 22904-4137, USA

E-mail address: rt7u@virginia.edu 


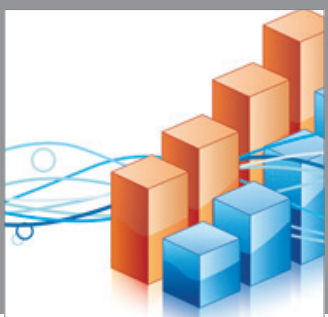

Advances in

Operations Research

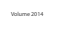

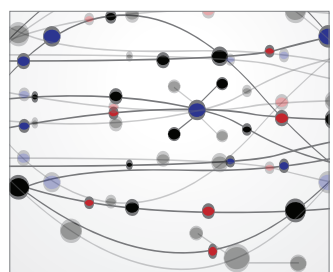

\section{The Scientific} World Journal
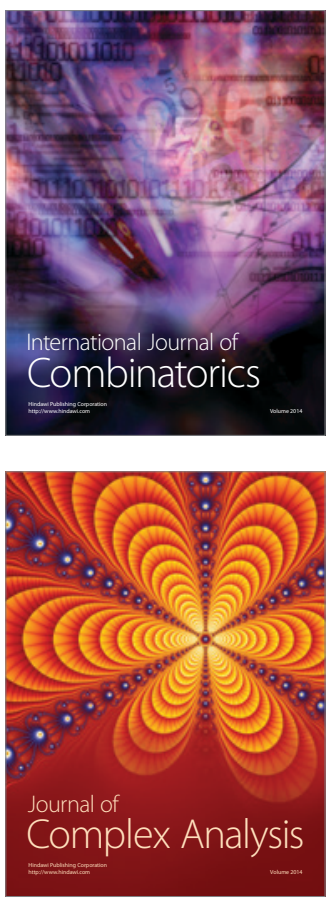

International Journal of

Mathematics and

Mathematical

Sciences
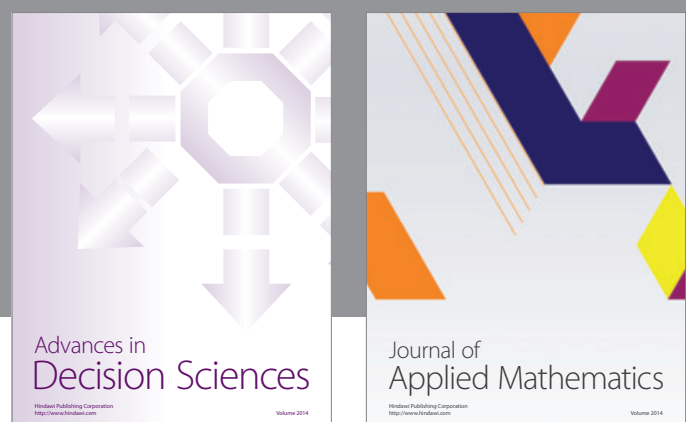

Journal of

Applied Mathematics
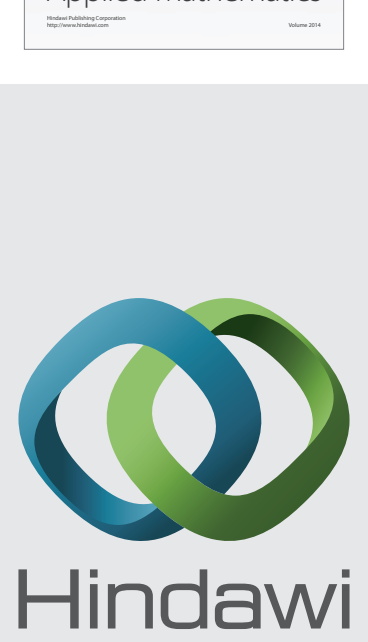

Submit your manuscripts at http://www.hindawi.com
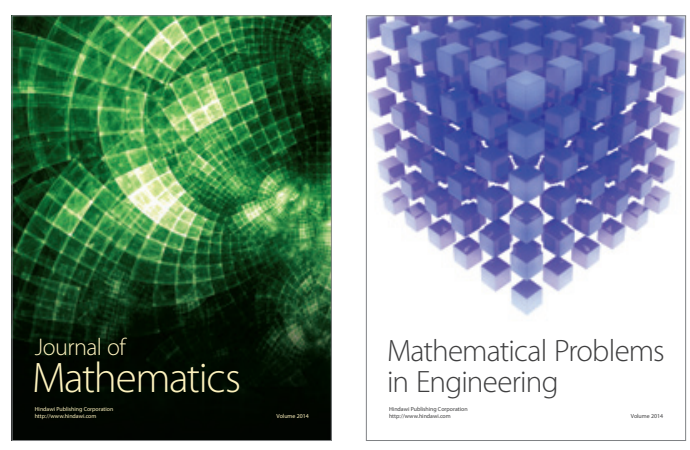

Mathematical Problems in Engineering
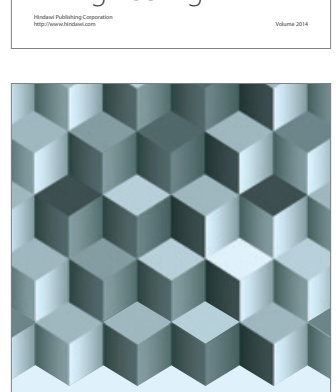

Journal of

Function Spaces
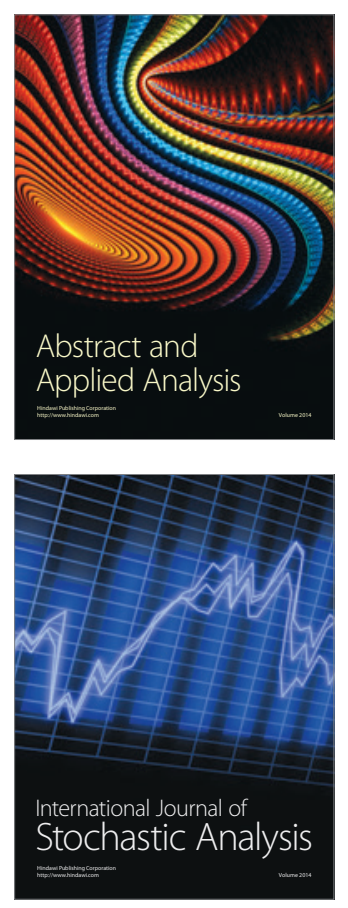

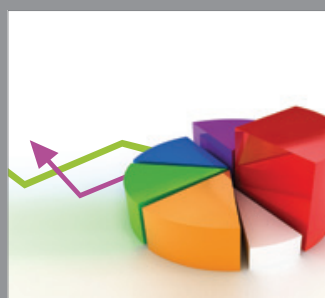

ournal of

Probability and Statistics

Promensencen
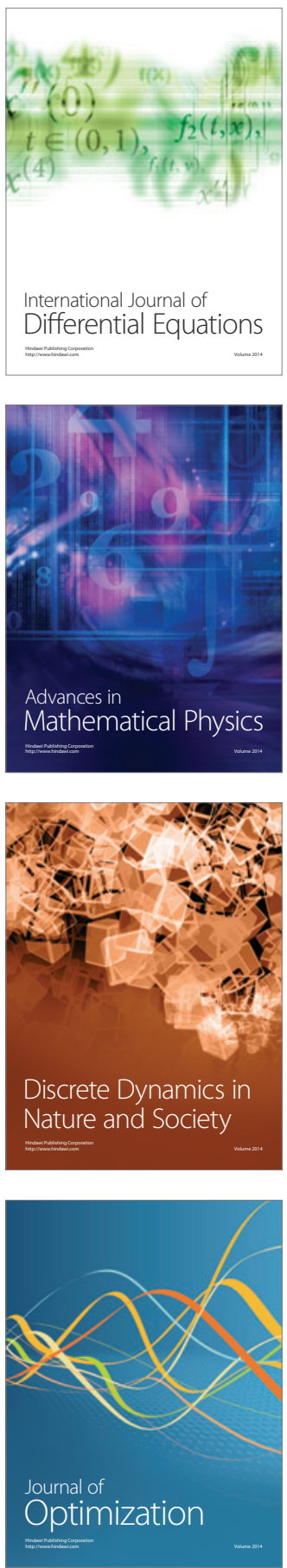